\title{
Transmission Electron Microscopy of 2D Materials: Structure and Surface Properties
}

\author{
Linda Karlsson
}


Cover: Multiple stacked $\mathrm{Ti}_{3} \mathrm{C}_{2} \mathrm{~T}_{x}$ MXene sheets imaged by low-voltage, monochromated, aberration-corrected transmission electron microscopy.

During the course of research underlying this thesis, Linda Karlsson was enrolled in Agora Materiae, a multidiciplinary doctoral program at Linköping University, Sweden.

(C) Linda Karlsson, unless stated otherwise ISBN 978-91-7685-832-5

ISSN 0345-7524

Printed by LiU-Tryck 2016 
I rarely end up where $I$ was intending to go, but often I end up somewhere I needed to be.

- Douglas Adams The Long Dark Tea-Time of The Soul 

During recent years, new types of materials have been discovered with unique properties. One family of such materials are two-dimensional materials, which include graphene and MXene. These materials are stronger, more flexible, and have higher conductivity than other materials. As such they are highly interesting for new applications, e.g. specialized in vivo drug delivery systems, hydrogen storage, or as replacements of common materials in e.g. batteries, bulletproof clothing, and sensors. The list of potential applications is long for these new materials.

As these materials are almost entirely made up of surfaces, their properties are strongly influenced by interaction between their surfaces, as well as with molecules or adatoms attached to the surfaces (surface groups). This interaction can change the materials and their properties, and it is therefore imperative to understand the underlying mechanisms. Surface groups on two-dimensional materials can be studied by Transmission Electron Microscopy (TEM), where high energy electrons are transmitted through a sample and the resulting image is recorded. However, the high energy needed to get enough resolution to observe single atoms damages the sample and limits the type of materials which can be analyzed. Lowering the electron energy decreases the damage, but the image resolution at such conditions is severely limited by inherent imperfections (aberrations) in the TEM. During the last years, new TEM models have been developed which employ a low acceleration voltage together with aberration correction, enabling imaging at the atomic scale without damaging the samples. These aberration-corrected TEMs are important tools in understanding the structure and chemistry of two-dimensional materials.

In this thesis the two-dimensional materials graphene and $\mathrm{Ti}_{3} \mathrm{C}_{2} \mathrm{~T}_{x}$ MXene have been investigated by low-voltage, aberration-corrected (scanning) TEM. High temperature annealing of graphene covered by residues from the synthesis is studied, as well as the structure and surface groups on single and double $\mathrm{Ti}_{3} \mathrm{C}_{2} \mathrm{~T}_{x}$ MXene. These results are important contributions to the understanding of this class of materials and how their properties can be controlled. 


\section{Populärvetenskaplig sammanfattning}

Tvådimensionella material har unika egenskaper som beror på deras speciella struktur. De lämpar sig därmed väl för framtidens elektronik som kräver dels kontroll ner på atomnivå och dels atomärt tunna material. Jag har undersökt dessa egenskaper med Linköpings universitets högupplösta transmissionselektronmikroskop, ett av de mest avancerade mikroskopen i världen.

För att kunna utveckla dessa material för praktiska tillämpningar behövs kunskaper om hur materialen ser ut och reagerar med sin närmaste omgivning. I min forskning har jag fokuserat på materialen grafen (uttalas grafén) och MXene. Grafens speciella egenskaper försämras kraftigt när ytan är täckt av plastrester från tillverkningsprocessen. För att kunna använda grafen i en färdig produkt krävs att den kan kopplas ihop med ett kontaktmaterial, vilket i dagsläget vanligtvis är en metall. MXene får olika egenskaper beroende på hur den tillverkas, vilket påverkar hur den sedan kan användas.

Det första och mest kända av de tvådimensionella materialen är grafen, som består av ett enda lager av kolatomer i ett hexagonalt mönster. Ett nyare tvådimensionellt material är MXene, som består av enstaka atomära lager av titan och kol i ett hexagonalt mönster. På grund av denna hexagonala struktur har materialen en närmast perfekt ledningsförmåga och är dessutom enkelt formbara. Grafen är mjukt men blir starkare ju större yta materialet har, och är därmed väl anpassat för t.ex. böjbara skärmar eller personlig skyddsutrustning, medan MXene utblandad i lite vätska kan bilda en formbar, elektriskt ledande lera, för till exempel nya typer av batterier.

I min forskning har jag studerat atomer och molekyler på grafens och MXenes ytor. Genom att lägga metallatomer på grafen och sedan hetta upp materialet kan jag se hur metallatomerna interagerar med plastrester på grafenet. Det visar sig att metallatomer växelverkar med plastrester även om de är placerade på olika sidor av grafenet. Detta kan ha en påverkan på grafenets egenskaper och därmed hur grafenet kommer att prestera i den slutliga produkten. På MXenet studerade jag den atomära strukturen hos materialet och de molekyler och atomer som fastnat 
på dess ytor under tillverkningsprocessen. Dessa molekyler och atomer påverkar MXenes egenskaper.

Jag studerade dessa material genom att använda ett av de mest avancerade mikroskopen i världen, ett så kallat linsfelskorrigerat transmissionselektronmikroskop. Detta mikroskop accelererar elektroner upp till väldigt höga hastigheter och skjuter dessa genom grafenet eller MXenet. De spridda elektronerna träffar sedan detektorer som direkt skapar en bild av det undersökta materialet. Normalt har transmissionselektronmikroskop inneboende linsfel, t.ex. sfärisk aberration som suddar ut detaljer i bilderna. Ett linsfelkorrigerat mikroskop minimerar dessa linsfel, och ökar därmed upplösningen i bilderna. Det gör att detta mikroskop kan avbilda enskilda atomer och avslöja de tvådimensionella materialens innersta egenskaper. 
This thesis is the result of research performed at the Thin Film Physics Division at the Department of Physics, Chemistry and Biology (IFM) at Linköping University, Sweden, between January 2011 and June 2016.

The research has focused on low-voltage, monochromated, aberration-corrected TEM studies of the structure and chemistry of two-dimensional materials, especially atoms and molecules present on the material's surfaces. The thesis is divided into two parts, the first part is focused on the interaction and evolution of plastic residues and metal oxide particles on graphene during in situ high temperature annealing, while the second part is focused on the structure and chemistry of single sheets of $\mathrm{Ti}_{3} \mathrm{C}_{2} \mathrm{~T}_{x}$ MXene.

The microscopy has been performed at the Electron Microscopy Laboratory at Linköping University and The EPSRC National Facility for Aberration Corrected STEM (SuperSTEM) in Great Britain. The research is presented in the form of research papers submitted to scientific journals, or as manuscripts which will be submitted to scientific journals.

The work has been supported by the Swedish Research Council (Vetenskapsrådet, VR) under grants no. 621-2012-4359, 622-2008-405, 621-2009-5294, and 642-2013-8020.

During my graduate studies I was awarded a travel grant from Ericsson's Research Foundation for participation in the Electron Microscopy Congress (EMC) 2012 and a travel grant from Ångpanneföreningens Forskningsstiftelse (ÅForsk) for participation in Annual Meeting of the Nordic Microscopy Society (Scandem) 2012. 


\section{Acknowledgements}

As many of my friends know, I am a huge fan of both fantasy and science fiction. My time as a PhD student has had many similarities with a fantasy or science fiction adventure; I've worked with elves and hobbits, fought with the first of men and conjured lightning ${ }^{1}$. It's taken a long time, and most of the time has been spent wondering what I am doing and why. I've tried to accomplish something which at certain moments appeared to be beyond impossible, but things worked out, although not in the way I or anyone else had foreseen. And just as the heroes in fictional adventures, I've had a lot of help to get me to the point I am today.

So here I've tried to list all those that have helped me during this adventure. I hope I have not forgotten anyone, if so I am very sorry.

\section{THANK YOU to:}

My supervisor, Per Persson, for giving me this opportunity, supporting me throughout the years and for introducing me to the black magic of TEM and TEM sample preparation. I'm amazed at how you found the energy to continue when I had to reboot. I love our discussions about anything and everything.

My co-supervisor, Jens Birch, for all your support and showing me the white magic of science and writing.

My co-authors for all samples, help, and support with the papers. Without you this thesis would just be a lot of blank pages.

Thomas, Harri, Kalle, Anette, Kirstin, and Malin for all your help with anything and everything. Nothing would have worked without you.

Firandegruppen, (Anders, Camilla, Christopher, David, Fredrik, Karin, Katarina, Kjersti, Lina, Ludvig, Mathias, Per, Pontus, Sara) for all the celebrations

\footnotetext{
${ }^{1}$ For those who are not Thin Filmers: Two of the transmission electron microscopes are named Galadriel and Arwen, the ion millers used for sample preparation Merry, Pippin and Bilbo, and the magnetron sputtering system I used for a while is named Adam, which could get electric discharges at the target.
} 
and fikas. We should definitely have more of them!

Kaffeklubben with support, (Jonas, Anders, Christopher, Fredrik, Jonas, Camilla, Patricia, Judit, Robert, Kalle, Robert, Per, Andreas, Sara, Pontus, Gunnar, Katarina, and Lina) for all weird and wonderful discussions during lunches and fikas.

The IFM PhD Reference Group, current and former members (Martin, Mattias, Lina, Katarina, Sit, Pia, Linda, Nina) for all fun times and long discussions.

The The Pub Group, (especially Daniel, Christopher, Pontus, Camilla) for all the help with getting the pubs going and for continuing to make them even better.

Everyone in the Board of LiUPhD, especially Fredrik and Viktor, for letting me be a part of this new adventure.

Everyone in Agora Materiae, for all the good times we've had. It's been great and it's really fun to see what happened since the first board meeting.

Justinas, for all your help with anything related to TEM and for trying to lift my spirit when I was feeling down. How you managed to stay so positive during your PhD studies I still don't understand.

Amie, for all the help and all the fikas during my first years here when we were both struggling to understand what we were doing.

The inventor of Tea, for realizing that putting leaves in hot water makes a beverage with awesome powers.

And, of course, my closest companions during these years:

Lina, my friend, support, and thesis-writing twin. I don't know how to express my gratitude for all the help and encouragement you have given me during these years. I would never have made it without you. Thank you.

Katarina, thank you for all the fikas, lunches, discussions, knitting evenings... It's amazing how much a cup of tea can help.

Patrik and Nina, the greatest surprise of moving here to Linköping was meeting you two. It's hard to find a kindred spirit, but finding two at the same time was amazing. All I can say is the cake wasn't a lie.

My family (Mamma, Pappa, Mia, Stoffer, Mormor, Morfar), thank you for always being there, even when I didn't understand that I needed it. Thank you for teaching me to never, ever give up.

Daniel, my very own Darth Vader, thank you for all support and believing in me when I didn't. Thank you for allowing me to do almost anything I want (except putting pink stuff everywhere). I love you.

Freddy Krueger, the small ball of fur and claws whom always reminded me of what is important in life: sleep, play, and grilled chicken.

Last but not least, I would like to thank Kacke. We are the best team ever. 
1 Introduction 1

1.1 Thin Films . . . . . . . . . . . . . . . . . . . . 1

1.2 Nanomaterials . . . . . . . . . . . . . . . . . 2

1.2.1 Zero-Dimensional and One-Dimensional Materials . . . . . 3

1.2.2 Two-Dimensional Materials . . . . . . . . . . . . . . 3

1.3 Aim of Thesis . . . . . . . . . . . . . . . . . . . . . . . . . . . . . . . . .

1.4 Outline of Thesis . . . . . . . . . . . . . . 5

2 Graphene 7

2.1 Structure ........................... 7

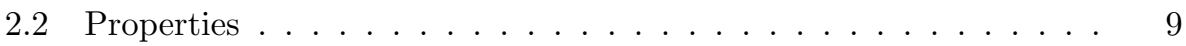

2.2.1 Tailoring Properties . . . . . . . . . . . . . 11

2.3 Synthesis . . . . . . . . . . . . . . . . . . . . . . . . . . . . . . . . . 12

2.3.1 Chemical Vapour Deposition . . . . . . . . . . . . 12

2.3.2 Transfer of Graphene . . . . . . . . . . . . . . . . . . . 13

2.3.3 Magnetron Sputtering . . . . . . . . . . . . . . . . . 14

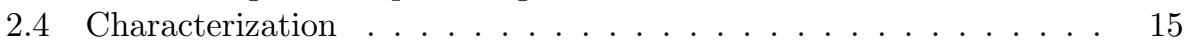

2.5 High Temperature Annealing . . . . . . . . . . . . . . 16

2.5.1 Dendritic Growth . . . . . . . . . . . 17

$\begin{array}{llr}3 & \text { MXene } & 19\end{array}$

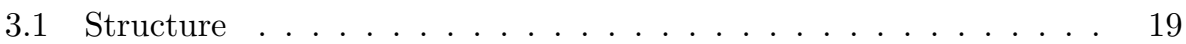

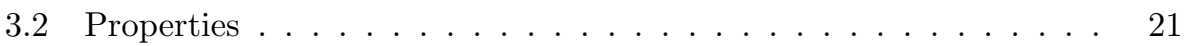

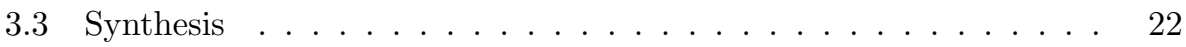

3.3.1 MAX Phase Materials . . . . . . . . . . . . . 22

3.3 .2 Selective Etching . . . . . . . . . . . . . . 23

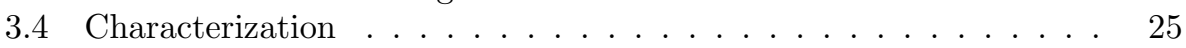

3.5 Surface Groups . . . . . . . . . . . . . . . . . 25

3.5.1 Effect on Properties .............. . . 27 
3.6 Intercalants . . . . . . . . . . . . . . . . . . . . . 27

3.6.1 Effect on Properties . . . . . . . . . . . . 28

4 Aberration-Corrected Transmission Electron Microscopy 29

4.1 The Transmission Electron Microscope . . . . . . . . . . . . . . . . 31

4.1 .1 Illumination System . . . . . . . . . . . . . . 32

4.1 .2 Imaging System . . . . . . . . . . . . . . . . . . . . . . . . . . . . . . . . 33

4.2 Main TEM Imaging Techniques . . . . . . . . . . . . . . . . . . . . . 33

4.2.1 Elastical Scattering . . . . . . . . . . . . . . . . . . . . . . . . . 33

4.2 .2 STEM Imaging . . . . . . . . . . . . . . . . . . . . . . 33

4.2 .3 CTEM Imaging . . . . . . . . . . . . . . . . . . . . . . . . . 35

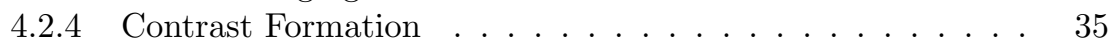

4.3 Aberrations in TEM . . . . . . . . . . . . . . . . . . 39

4.3.1 Chromatic aberration $\left(C_{c}\right)$. . . . . . . . . . . . . 39

4.3.2 Spherical Aberration $\left(C_{3,0}, C_{3}\right.$, or $\left.C_{s}\right) \ldots \ldots 40$

4.3.3 Astigmastim $\left(C_{1,2}\right.$ or $\left.A_{2}\right) \ldots \ldots$. . . . . . . . 40

4.3.4 Coma $\left(C_{2,1}\right.$ or $\left.B_{2}\right) \ldots \ldots \ldots 41$

4.3.5 Correcting Aberrations ................ 41

4.3.6 Stability of Aberration-Corrected TEM . . . . . . . . . 42

4.4 Main Analytical TEM Techniques . . . . . . . . . . . . . . 43

4.4 Inelastic Scattering . . . . . . . . . . . . . . . . . . 44

4.4.2 Electron Energy Loss Spectroscopy (EELS) . . . . . . . . . 44

4.4 .3 Spectrum Imaging . . . . . . . . . . . . . . . . . . . . . . 47

4.5 Radiation Damage . . . . . . . . . . . . . . . . . . . . . . 48

4.5.1 Knock-on Displacement . . . . . . . . . . . . . . . . 48

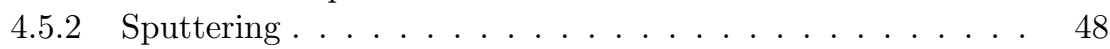

4.5.3 Heating ............................. 49

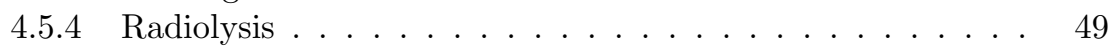

4.5.5 Limiting Radiation Damage . . . . . . . . . . . . . 49

5 Conclusions $\quad \mathbf{5 1}$

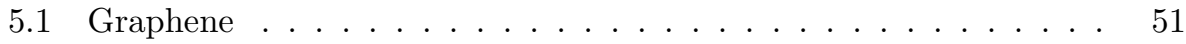

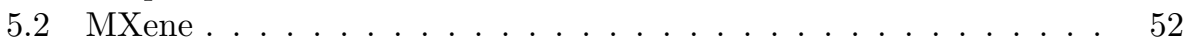

6 Future Outlook 53

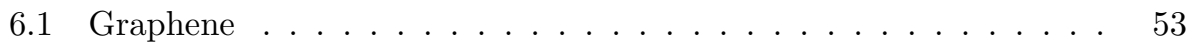

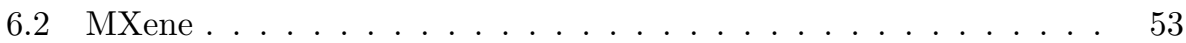

Bibliography $\quad 55$

7 Publications Included in the Thesis $\quad 69$

8 Summary of Included Papers $\quad 71$

9 Publications Not Included in the Thesis $\quad 75$

$\begin{array}{ll}\text { Paper I } & 77\end{array}$ 
$\begin{array}{lr}\text { Paper II } & 85\end{array}$

$\begin{array}{ll}\text { Paper III } & 91\end{array}$

$\begin{array}{ll}\text { Paper IV } & 109\end{array}$

$\begin{array}{lr}\text { Paper V } & 121\end{array}$

$\begin{array}{lc}\text { Appendices } & 133\end{array}$

$\begin{array}{lc}\text { A List of Figures } & 133\end{array}$

$\begin{array}{ll}\text { B List of Tables } & 137\end{array}$

$\begin{array}{ll}\text { C Abbreviations } & 139\end{array}$ 


\section{CHAPTER 1}

\section{Introduction}

Bender: Hey, Flatso, how do we get out of this two-bit dimension?

Prof. Farnsworth: There's a simple answer for that. We can't. But on the upside, we've got a whole dimension to explore with entirely different laws of physics. Why, watch what happens when I drop this marble. Well, that's the same, but other things are different.

Futurama, Season 7, Episode 14 (2-D Blacktop) 2013

Computers, mobile phones, video game consoles, credit cards, and the internet are all examples of objects that are of great importance to the modern way of life. These are results of the technological advancement during the last decades, which has been facilitated by the development of new materials. This development is a part of materials science, a research field devoted to the understanding and improvement of existing materials as well as the discovery of new materials with novel properties. Materials science include synthesis and characterization of materials, as well as theoretical predictions of materials and their properties. A part of materials science is the research on thin films and nanomaterials, in which the material size is restricted in at least one dimension. Research on such materials have helped to fulfill Moore's law, predicted by Gordon E. Moore in 1965 [1], stating that approximately every second year the number of transistors in an integrated circuit doubles, creating more powerful computers as well as smaller electronics.

\subsection{Thin Films}

Thin films are materials with thickness below a fraction of the thickness of a human hair (which is $\sim 100 \mu \mathrm{m}$ ) with properties that may differ from the bulk, 
such as different colours, higher hardness or higher conductivity. An example of a thin film which increases the hardness of a material is shown in Figure $1.1^{1}$. The first known use of thin films is from ancient Egypt, around 3000 BC, as decorative gold coatings [2]. From the 19th century, thin films have been developed also as coatings for electronic or protective applications. Thin films are usually synthesized on the surfaces of bulk materials, enhancing the properties of the bulk or providing complementary properties. Examples are carbon-based coatings for protection of stored data in hard drives [3], metal alloy coatings on Blu Rays [4], and anti-reflective coatings on eyeglasses.

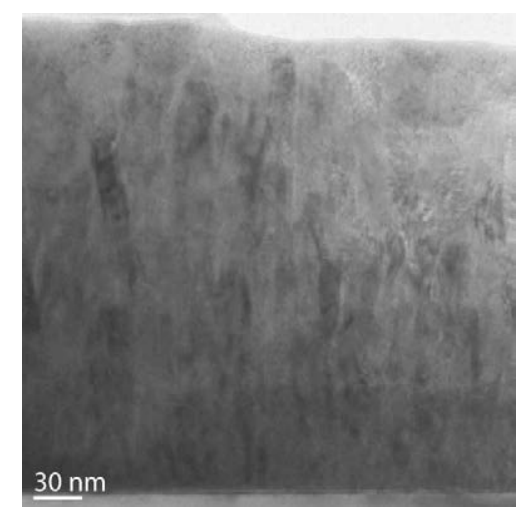

Figure 1.1. Example of a thin film.

\subsection{Nanomaterials}

Nanomaterials are materials where at least one external dimension or the internal structure or surfaces are in the range of $\sim 1-100 \mathrm{~nm}$ [5]. This may change the properties of the materials from those of the bulk towards those of the surfaces. Examples of nanomaterials are thin films, particles, nanorods, nanowires, aggregates (strongly bound particles), and agglomerates (loosely bound particles). Nanomaterials have become more important and common during the last decade in e.g. electronics such as computer hardware, computer screens, TV's, and batteries, but also hygiene products such as sunscreens [6] and odor-reducing clothes [7]. If the motion of electrons or protons are restricted, nanomaterials are denoted as zero-, one-, or two-dimensional depending on in how many dimensions the motion is restricted.

\footnotetext{
$\mathrm{Lu}$.

${ }^{1} \mathrm{The} \mathrm{ZrB}_{2}$ film was synthesized by Lina Tengdelius and the TEM image was acquired by Jun
} 


\subsubsection{Zero-Dimensional and One-Dimensional Materials}

Zero-dimensional materials have restrictions in all dimensions, creating particles with a diameter of a few $\mathrm{nm}$. The best known examples are the C-60 molecule [8], see Figure 1.2a, and quantum dots. Similarly, one-dimensional materials are restricted in two dimensions and appear as rods or tubes, therefore usually denoted nanorods or nanotubes, see Figure $1.2 \mathrm{~b}^{2}$. Due to their optical properties, quantum dots and nanorods are promising materials for optical applications such as Light Emitting Diodes (LEDs) $[9,10,11]$.
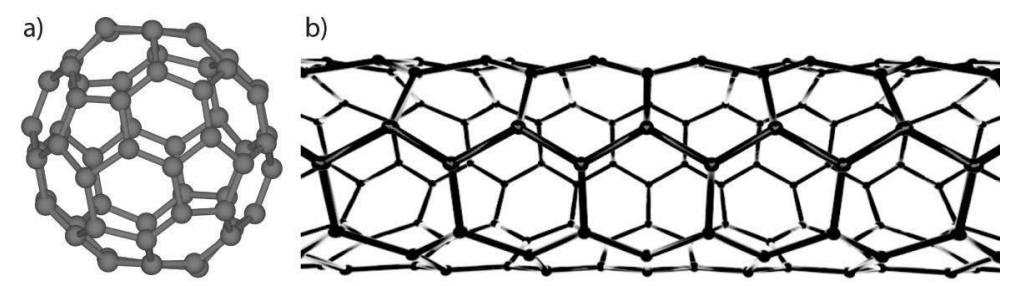

Figure 1.2. Schematic of a zero-dimensional C-60 molecule (a) and a one-dimensional carbon nanotube (b).

\subsubsection{Two-Dimensional Materials}

Two-dimensional materials have a thickness between one atomic layer and a few $\mathrm{nm}$, and electron movements are restricted in the out-of-plane direction (z-direction) [12], see Figure 1.3. The figure shows schematics of two examples of two-dimensional materials: graphene (Figure 1.3a-b), consisting of a single layer of carbon atoms, and $\mathrm{Ti}_{3} \mathrm{C}_{2}$ MXene (Figure 1.3c-d), consisting of five atomic layers of alternating titanium and carbon. Observing the sheets in a direction parallel to the atomic planes (side view) shows the atomic layering and the restriction in z-direction, while observing from a direction perpendicular to the atomic planes (top view) shows the unrestricted, periodic structure of the sheets.

Even though the existence of two-dimensional materials have been theorized since the 1940's [13], it was not until 2004 that it was shown that these materials can be stable as freestanding sheets, by the isolation of individual graphene sheets [14]. This caused an immense interest in two-dimensional materials and a wide range of these have since been synthesized [15, 16, 17, 18, 19, 20, 21, 22, 23], or predicted $[24,25]$. Prominent examples of two-dimensional materials are graphene [14], MXene [16], Transition Metal Dichalcogenides (TMDs) (e.g $\mathrm{WS}_{2}, \mathrm{MoS}_{2}$ ) [17], and boron nitride (BN) [26].

\footnotetext{
${ }^{2}$ Figure $1.2 \mathrm{~b}$ ) is based on Metallic nanotube.png from Wikimedia Commons. The copyright was released to the public domain in 2007. https://commons.wikimedia.org/wiki/File:Metallic _nanotube.png
} 

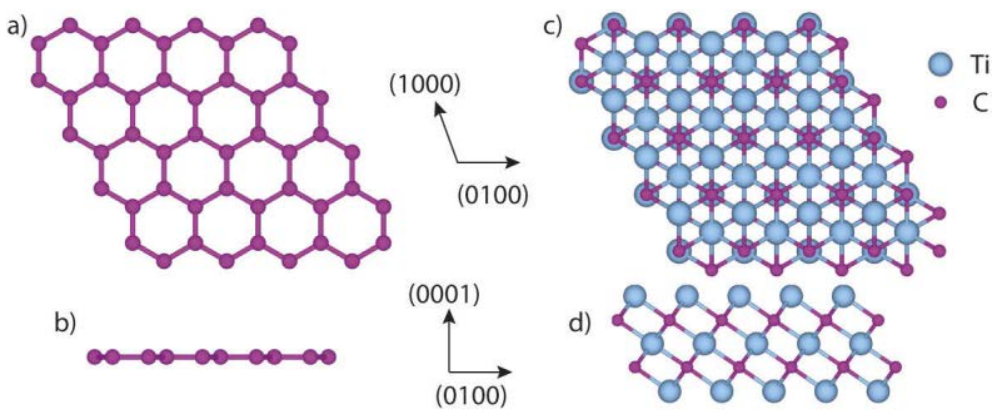

Figure 1.3. Schematic of the two-dimensional materials graphene in top view (a) and side view (b), and $\mathrm{Ti}_{3} \mathrm{C}_{2}$ MXene in top view (c) and side view (d).

\section{The Significance of Surfaces}

The high interest in two-dimensional materials arise from their unique properties, a result from their structure and chemistry being determined almost solely by their surfaces and by the restriction of electron motion to two-dimensions. As two-dimensional materials have a uniquely high surface-to-weight ratio, they have a much higher reactivity per weight unit than other materials.

Examples of other properties of two-dimensional materials are combined brittleness and ductility [27], very high conductivity [14, 15, 28], non-permeability to any kind of atom [29], and room temperature quantum phenomena, observed in other materials at temperatures at very low temperatures $[27,30]$. These properties give the possibility of many new applications such as specialized in vitro drug delivery systems [31], foldable screens [20], and hydrogen storage [29] to name but a few. The high surface-to-weight ratio makes two-dimensional materials excellent candidates for applications dependent on surface reactions, such as catalysts [32] and electrochemical capacitors (batteries) [33].

Apart from the new, unique properties of two-dimensional materials, they also present new research challenges. As they consist almost entirely of surfaces, their properties depend strongly on the atoms and molecules attaching to the surfaces $[16,27,34]$. Some of these surface groups affect the properties to such an extent that they are perceived as new two-dimensional materials, such as graphene oxide [35] or graphane (graphene covered with hydrogen) [24]. The surface groups may even severely damage the material [36].

As the surface groups change the material's properties, they are often referred to as functional groups, and the two-dimensional material as functionalized materials. Functional groups are small molecules or atoms attached to a larger molecule, and cause the characteristic reactions of that material. A known example is iron in hemoglobin in our blood, to which oxygen is attached and released at specific points in our bodies. Example of functional groups on a two-dimensional materials are oxygen-based molecules which change graphene from hydrophobic to hydrophilic [34]. Employing functional groups makes two-dimensional materials interesting for sensing applications [37] and as lithium support in batteries [38, 39] 
instead of cobalt, nickel or manganese-salts commonly employed [40].

Surface groups attach to the two-dimensional materials during the manufacturing process, handling, storage or chemical treatments. It is imperative to understand how these surface groups and two-dimensional materials interact, so the properties can be controlled. One powerful method of investigating twodimensional materials and their surface groups is TEM. A Transmission Electron Microscope (TEM) transmits electrons with energies normally between $60-300 \mathrm{keV}$ through a sample and detect the changes of electron paths and energies. In 1932 the first TEM was constructed, achieving a magnification of $17.4 \mathrm{x}$ [41] and a resolution (observable separation of two points) slightly better than an optical microscope [42]. Electron microscopes have since then been improved continuously and a standard TEM can today magnify $>10^{6} \mathrm{x}$ and resolve points separated by $\sim 2 \AA$, which provides information regarding the crystal structure within a sample. As the electrons employed in a TEM can ionize atoms and break chemical bonds, radiation damage is often observed during TEM analysis. The latest generations of TEM can image and analyze single atoms at a resolution below $1 \AA$ with minimal damage to the sample.

\subsection{Aim of Thesis}

The aim of this thesis is to study the structure and surface properties of the two-dimensional materials graphene and $\mathrm{Ti}_{3} \mathrm{C}_{2} \mathrm{~T}_{x}$ MXene (where $\mathrm{T}_{x}$ denote the surface groups) by low-voltage, monochromated, aberration-corrected TEM. As two different materials have been studied, the thesis is divided into two parts:

1. Behavior of transfer residues on the surface of freestanding graphene during in situ annealing.

2. Structure of and surface groups on single $\mathrm{Ti}_{3} \mathrm{C}_{2} \mathrm{~T}_{x}$ MXene sheets.

\subsection{Outline of Thesis}

This first chapter is an introduction to the field of two-dimensional materials, while the second chapter discusses graphene and how residues present on its surface change during in situ annealing. The third chapter discusses the structure and surface groups on $\mathrm{Ti}_{3} \mathrm{C}_{2} \mathrm{~T}_{x}$ MXene. Both chapters discuss structure, properties, and synthesis of the materials. In the fourth chapter the principle of low-voltage, monochromated, aberration-corrected TEM is explained. At the end, the five papers which are the basis for the thesis is presented, with an explanation of my contribution to each paper. 


\section{CHAPTER 2}

\section{Graphene}

God made the bulk

Surfaces were invented by the devil

Wolfgang Pauli

Graphene consists of a two-dimensional, hexagonal network of carbon atoms. Carbon is one of the most abundant elements in the universe, originating from fusion of helium in stars. On Earth, it is one of the key elements in sustaining life and is among the first known elements. Its importance for life and for the development of human civilization originate from its ability to bond to almost all elements and itself in many different ways, providing a large diversity of materials and material properties. Carbon fibers, graphite, and diamond are examples of carbon-based materials with very different properties. These differences arise from variations in structure and chemical bonds, which changes the interaction between atoms and electrons.

\section{$2.1 \quad$ Structure}

When bonded to itself, carbon can be found in many different allotropes, materials with different bonding, see Figure $2.1^{1}$ for examples. The best known carbon allotropes are graphite, amorphous carbon (carbon with disordered atomic positions, found e.g. partially in coal), and diamond. Graphene and fullerenes (e.g. C-60 molecules and nanotubes) are more recently discovered allotropes. These different allotropes occur due to differences in the organization of chemical bonds,

\footnotetext{
${ }^{1}$ Figure $2.1 \mathrm{~g}$ ) is based on Metallic nanotube.png from Wikimedia Commons. The copyright was released to the public domain in 2007. https://commons.wikimedia.org/wiki/File:Metallic nanotube.png
} 

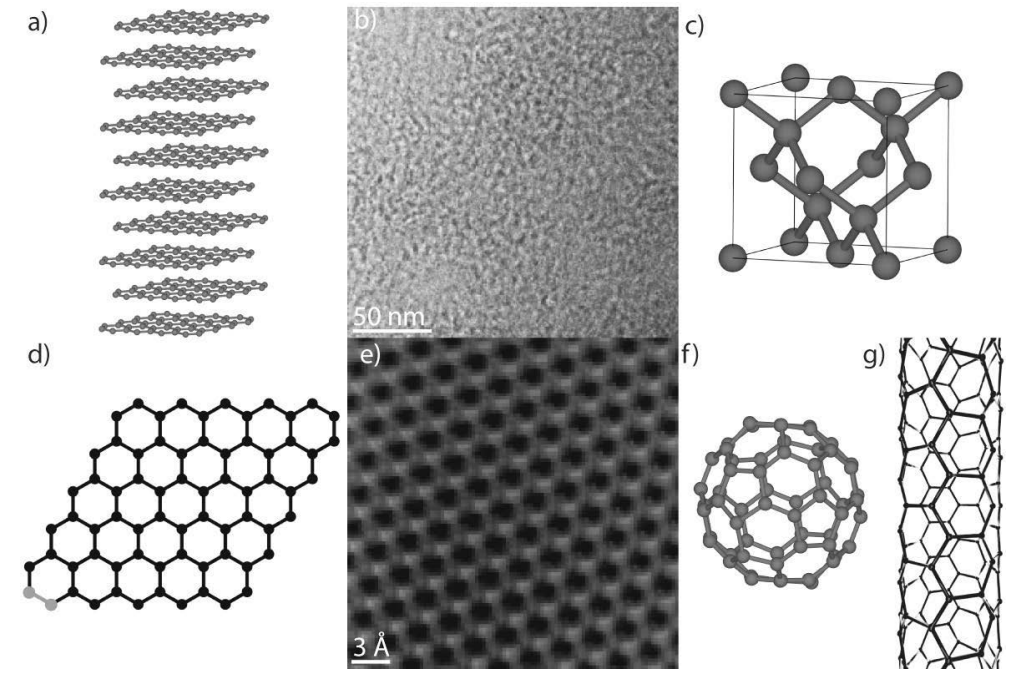

Figure 2.1. The most common carbon allotropes: graphite (a), amorphous carbon (b), diamond (c), graphene (d,e), C-60 (f) and nanotube (g). (c) and (e) are TEM images, where (e) is filtered and has inverted contrast to simplify interpretation.

where the most stable bond appear in the hexagonal structure of graphene and graphite, see Figure 2.1a,d,e. Despite the popular phrase "Diamonds are forever" 2 , diamond is a metastable phase and converts into graphite at a negligible rate. In the diamond lattice one carbon atom is bonded to four other, creating a Face Centered Cubic (FCC) structure commonly known as the diamond structure, as seen in Figure 2.1c. Amorphous carbon consist of a random network of carbon atoms, and usually also contains hydrogen which attach to dangling bonds (bonds not attached to an atom in the network). Amorphous carbon can also graphitize, for instance during electron irradiation. This can be observed in a TEM as amorphous carbon is commonly used as a supporting material for powders and nanorods.

Graphene consists of a single layer of carbon atoms arranged in a hexagonal lattice (see Figure 2.1d-e). The graphene unit cell consists of two atoms, marked in grey in Figure 2.1d, with an in-plane lattice constant of $2.46 \AA$. Stacking graphene sheets introduces a weak electron exchange between sheets (van der Waals interaction), which changes graphene's properties to become more like those of graphite. To distinguish the different types of graphene and their properties, stacked graphene is named differently depending on number of layers. Bilayer graphene (2 layers) have properties similar to graphene, while few-layer graphene (3-5 layers) and multilayer graphene (6-9 layers) have properties more close to graphite, which consist of ten or more layers [43]. The spacing between sheets in graphite is $3.35 \AA$ [44] and the sheets are stacked so that one carbon atom is positioned above the center of a hexagon, see Figure 2.2a-b. This is known as Bernal stacking or AB-stacking. In bilayer and few-layer graphene the stack-

\footnotetext{
${ }^{2}$ A James Bond movie from 1971, produced by Eon Productions Limited
} 


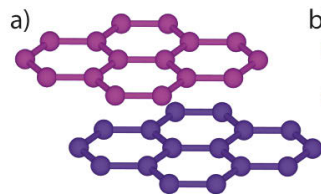

b)

c)
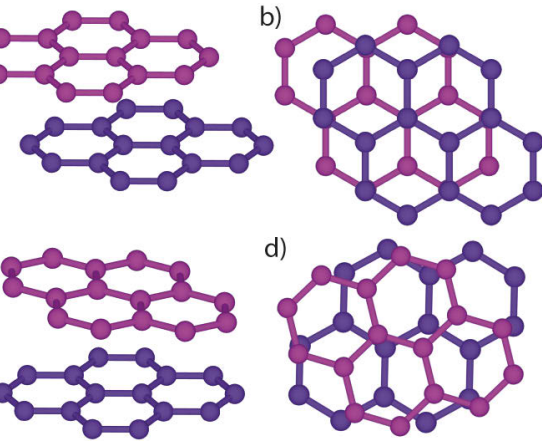

d)

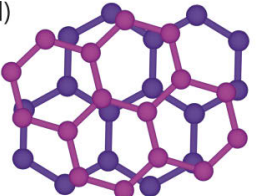

Figure 2.2. Schematic of bernal stacking of graphite in side view (a) and top view (b), and turbostratic stacking of bilayer graphene in side view (c) and top view (d).

ing may differ from $\mathrm{AB}$ stacking, as the growth conditions influence interaction between sheets. This may cause the sheets to be rotated randomly, known as turbostratic graphene [45], see Figure 2.2c-d. Turbostratic graphene may have an increased sheet separation, as the interaction between sheets is decreased.

\subsection{Properties}

Due to the differences in structure, carbon allotropes have a wide range of properties, see Table 2.1. Diamond is transparent and colorless, graphite is opaque and range from black to grey. Graphene is nearly transparent, transmitting $97.7 \%$ of incident light, decreasing linearly with increasing number of layers [44]. The color of carbon nanotubes depends on the radius [46] and films of carbon nanotubes can be made to absorb $99.055 \%$ of incident light, resulting in the blackest material yet [47].

Table 2.1. Comparison of properties of some of the carbon allotropes.

\begin{tabular}{lllll}
\hline Property & Diamond & Graphite & Graphene & Carbon Nanotubes \\
\hline Optical & Transparent, colorless & Opaque, black-grey & Nearly transparent & Depending on radius \\
Elastic modulus $[\mathrm{TPa}]$ & $1.2[48]$ & $1.06^{3}[49]$ & $1.0[50]$ & $1.25[51]$ \\
$\begin{array}{l}\text { Thermal } \\
\text { conductivity }[\mathrm{W} / \mathrm{mK}]\end{array}$ & $1,000[52]$ & $150[52]$ & $5,000[53]$ & $6,600[54]$ \\
$\begin{array}{l}\text { Electrical } \\
\text { conductivity }\end{array}$ & insulator & semimetallic & semiconductor & metallic or \\
$\begin{array}{l}\text { Electron } \\
\text { mobility }\left[\mathrm{cm}^{2} / \mathrm{Vs}\right]\end{array}$ & $4,500[55]$ & $3,000^{5}[56]$ & $100,000[14]$ & semiconductor ${ }^{4}$ \\
\hline
\end{tabular}

\footnotetext{
${ }^{3}$ Limited by van der Waals interaction

${ }^{4}$ Depending on structure

${ }^{5} \mathrm{At}-196{ }^{\circ} \mathrm{C}$
} 
Comparing the mechanical properties, diamond is one of the hardest and stiffest materials known, with an elastic modulus of $1.2 \mathrm{TPa}$ [48], which can be compared with steel's $81 \mathrm{GPa}$ [52]. The values for graphene and carbon nanotubes are similar to diamond, at 1.0 TPa [50] and 1.25 TPa [51] respectively. Graphite has also similar hardness, but is limited by the weak interaction between sheets [49]. Due to their crystal structures, diamond is one of the least compressible materials known [58], while graphene is one of the most elastic [50]. The combination of hardness and elasticity makes graphene one of the strongest materials to date, and it can be stretched up to $20 \%$ [20].

Also the thermal and electrical conductivities varies between the different carbon allotropes. Diamond is a good thermal conductor $(1000 \mathrm{~W} / \mathrm{mK}[52])$, graphite is a poor thermal conductor $(150 \mathrm{~W} / \mathrm{mK}[52])$, while carbon nanotubes and graphene have unusual high thermal conductivities $(6600 \mathrm{~W} / \mathrm{mK}$ [54] and $5000 \mathrm{~W} / \mathrm{mK}[53])$. With regard to electrical conductivity, diamond is an insulator, graphene is an excellent conductor [14], graphite is an semimetal [59] and carbon nanotubes may be metallic or semiconducting depending on its structure [60].

Apart from these differences, graphene has been reported to have properties not previously seen in other materials. These include electron densities about one million times larger than reported for copper, the highest reported electron mobility [20], quantum Hall effect at room temperature [44], and impermeability to any gas (including hydrogen) [20]. For the first experiments confirming the unique properties of graphene, Andre Geim and Konstantin Novoselov was awarded the Nobel Prize in Physics in 2010 [61].

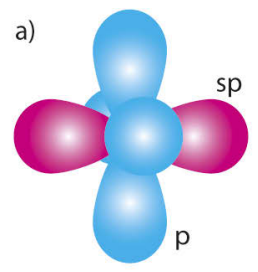

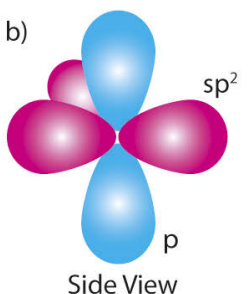
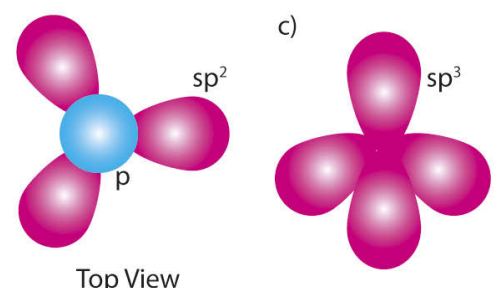

Figure 2.3. The three different carbon bonds where the hybridized orbitals are shown in pink and the non-hybridized are shown in cyan; sp found in acetylene (a), $\mathrm{sp}^{2}$ in graphene (b), and $\mathrm{sp}^{3}$ in diamond (c).

The large variation in properties of carbon allotropes arise from the difference in chemical bonds of the structures. As carbon consist of 6 protons and electrons, its ground state contains four electrons in the s orbitals and two electrons in the $\mathrm{p}$ orbital $\left(1 \mathrm{~s}^{2} 2 \mathrm{~s}^{2} 2 \mathrm{p}^{2}\right)$. As carbon form bonds in a structure, the $\mathrm{s}$ and $\mathrm{p}$ orbitals combine (hybridize) into three modes: $\mathrm{sp}, \mathrm{sp}^{2}$ and $\mathrm{sp}^{3}$, see Figure 2.3 . In the sp bond the s orbitals hybridize with one of the three $\mathrm{p}$ orbitals, creating two sp orbitals and leave two p orbitals, see Figure 2.3a. The sp bond occur in unsaturated hydrocarbons, e.g. acetylene. In the $\mathrm{sp}^{2}$ bond the s orbitals hybridize with two $\mathrm{p}$ orbitals, creating three $\mathrm{sp}^{2}$ orbitals and one $\mathrm{p}$ orbital. The three $\mathrm{sp}^{2}$ orbitals lie in the same plane, while the p orbital extends out perpendicular to the surface, 
see Figure 2.3b. This is the bond present in graphene and which gives graphene its unique properties. The $\mathrm{sp}^{2}$ orbitals form covalent bonds ( $\sigma$ bonds), while the extending p orbitals combine into a valence band $(\pi)$ and conduction band $\left(\pi^{*}\right)$. The $\pi$ and $\pi^{*}$ bands have a linear energy-momentum dispersion, see Figure 2.4.

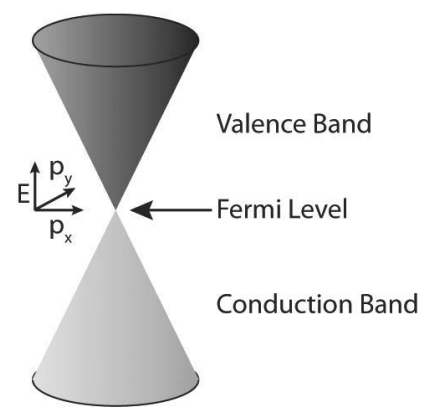

Figure 2.4. Schematic of the electronic structure of graphene with the position of the Fermi level indicated.

In a regular semiconductor the valence band and conduction band have a parabolic dependence. This difference causes the electrons in graphene to behave as massless Dirac fermions, instead of as normally, fermions described by the Schrödinger equation, in which the electrons have mass [44]. The shape of the conduction and valence bands makes graphene a zero-gap semiconductor.

In diamond one carbon atom is bonded to four other carbon atoms, causing the lowest energy state for the electrons to be a linear combination of $\mathrm{s}$ and $\mathrm{p}$ states, called $\mathrm{sp}^{3}$ (or $\sigma$ state), see Figure 2.3c.

Amorphous carbon has a random structure, with a combination of $\mathrm{sp}, \mathrm{sp}^{2}$ and $\mathrm{sp}^{3}$ bonds, where the ratio of $\mathrm{sp}^{2} / \mathrm{sp}^{3}$ bonds can be tuned.

\subsubsection{Tailoring Properties}

The properties of a material, depends strongly on variations in structure and chemistry of the material. As an example, doping diamond with boron turns it blue [62], while introducing vacancies changes its color to brown [63]. Changes in properties are also observed in graphene, where the most apparent changes are to its conductivity. Introducing metal dopants (e.g. aluminium, silver, copper) shift the Fermi level so graphene becomes either a p-type or a n-type semiconductor [64], while doping with e.g. phosphorous open up a band gap [65]. Dopants can also make graphene insulating [44] or introduce lattice distortions [66]. Lattice distortions such as stress and wrinkles are also caused by mismatching lattice constants between graphene and the substrate, observed in graphene on e.g. $\mathrm{SiC}$ [67], nickel [68], and copper [69]. The interaction between graphene and the substrate also changes the electronic properties, as $\mathrm{SiC}$ opens up a band gap [70] and limits the conductivity, similar to $\mathrm{SiO}_{2}$ [20]. The conductivity also changes with surface functionalization. Graphene oxide (graphene covered by hydroxide $(\mathrm{OH})$ and epoxy groups) can be tailored to become either insulating, semiconducting, 
or semimetallic [71]. Surface functionalization can also be used to detect minute traces of e.g. $\mathrm{CO}_{2}$ [72], $\mathrm{H}_{2}$ [73], or $\mathrm{NO}$ [74].

The possibility of intentionally tailoring graphene's properties makes it is an interesting material for a range of different applications, from transistors, touch screens, lasers, chemical sensors, to bulletproof vests [75]. However, during synthesis and processing, atoms and molecules unintentionally attach to graphene and compromise its properties. These residues may be more or less easy to remove, and thus the choice of synthesis process and handling is very important.

\subsection{Synthesis}

The first production method of graphene, reported by Novoselov et al. [14], was mechanical exfoliation of graphite using scotch tape. This produces graphitic flakes with a range of thicknesses from single sheets to multilayer graphene, where the crystal size depends on the crystal size of the original graphite. Even though it is an easy method to produce graphene, it is not suited for commercial production due to the limitation of crystal size, yield, and production rate. Thus other methods have been developed, such as high temperature sublimation of SiC, a semiconductor widely used in technological applications [76]. Annealing $\mathrm{SiC}$ at $1100-1150{ }^{\circ} \mathrm{C}$ causes silicon to sublimate from all $\mathrm{SiC}$ surfaces, leaving carbon atoms which rearrange into the thermodynamically most stable configuration, i.e. graphene [76]. By this method graphene is directly nucleated on an appropriate substrate. The graphene crystal size is here limited by the size of terraces on the $\mathrm{SiC}$ surfaces [77]. A more common method of graphene production is Chemical Vapour Deposition (CVD), which can synthesize good quality graphene up to $100 \mathrm{~m}$ long [78]. Graphene grown by CVD can be transferred between substrates, see Section 2.3.2, but this introduces residues onto the graphene, which cause defects and change the properties of graphene [79, 80, 81]. In Paper I and Paper II, residues remaining on graphene after transfer from copper to TEM grids were investigated.

\subsubsection{Chemical Vapour Deposition}

CVD is based on the decomposition and chemical reactions of one or several precursor gases near the surface of a substrate, often at high temperatures, see Figure 2.5. For growth of graphene, methane $\left(\mathrm{CH}_{4}\right)$ is used as a precursor and a metal is used as a substrate. Methane is inserted into a growth chamber together with a carrier gas, for the graphene investigated in this thesis a mixture of argon and hydrogen is used. Metals are preferred as substrates as they do not form carbides or solid solutions with carbon at the growth temperatures, which range from room temperature up to $\sim 1000{ }^{\circ} \mathrm{C}[82]$. During synthesis, carbon diffuses into the metal and precipitates onto the surface, where it forms into graphene. Copper is the most common substrate, but other metals have been used such as nickel [83], aluminium [84], and even liquid gallium [85].

Although graphene can be grown on copper, it is not a suitable substrate for graphene applications or for characterization in TEM. Thus graphene is normally 


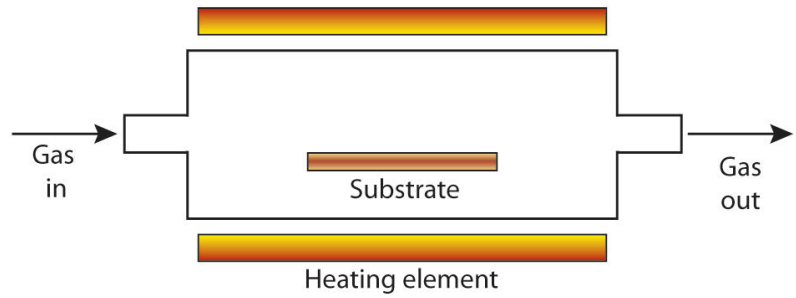

Figure 2.5. Schematic of the CVD growth chamber where the heater encircles the chamber.

transferred to more suitable substrates, such as $\mathrm{SiO}_{2}$ or TEM grids.

\subsubsection{Transfer of Graphene}

During transfer of graphene between substrates, the sheet needs to be supported to avoid wrinkles and cracks. Polymers (plastics) are used as support as they are easy to apply onto the graphene surface and can be easily removed. The most common is poly(methyl methacrylate) (PMMA). PMMA is a commonly used polymer, more known as acrylic glass or Plexiglass. It is transparent and is used as e.g. shatter-resistant glass [86].

As a support, PMMA is normally spin-coated onto graphene and the growth substrate is subsequently removed by etching, see Figure 2.6. The graphene sheet is then placed on a new substrate or a TEM grid, and the PMMA is removed by immersion in acetone. This dissolves most of the PMMA but leaves the graphene intact. After rinsing in deionized water, graphene is annealed in vacuum at $\sim 300{ }^{\circ} \mathrm{C}$ to remove more of the remaining PMMA [79, 87, 88]. However, a lot of PMMA residues are still present on the surface of the graphene. To remove these residues, graphene is annealed in an $\mathrm{H}_{2} / \mathrm{Ar}$ atmosphere, which causes the PMMA residues to decompose [89]. However, multiple studies have shown that PMMA residues and metal oxide particles from the metal substrate are left on graphene after transfer and subsequent annealing [79, 81, 87, 90, 91, 92]. This is illustrated in Figure 2.7, which show a Scanning Transmission Electron Microscopy (STEM) High-Angle Annular Dark Field (HAADF) image (STEM HAADF is described in Section 4.2.2) of a transferred graphene sheet. From the image it is clear that PMMA residues cover the entire surface, and there is a large amount of metal ox-

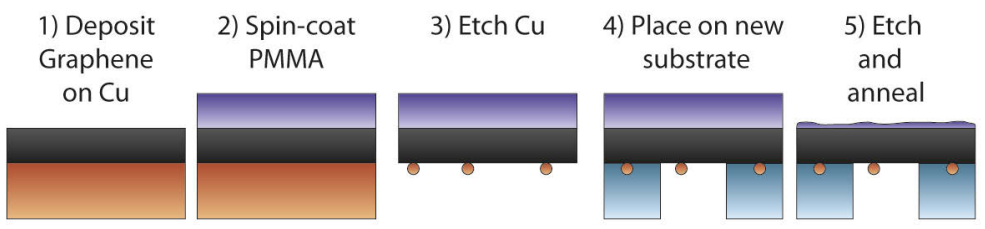

Figure 2.6. The transfer process of graphene between substrates. 
ide particles from the growth substrate. Studies of PMMA residues on graphene have shown that the graphene is p-doped [79], wrinkled [93], exhibit tears [94] and has limited conductivity [79]. In Paper I the interaction between PMMA residues and metal oxide particles remaining after synthesis and transfer was investigated. In Paper I, different amount of chromium atoms was deposited onto the graphene sheet after transfer to a TEM grid, to study how the amount of metal oxide particles affect the removal of PMMA residues. The chromium was deposited onto the graphene by magnetron sputtering.

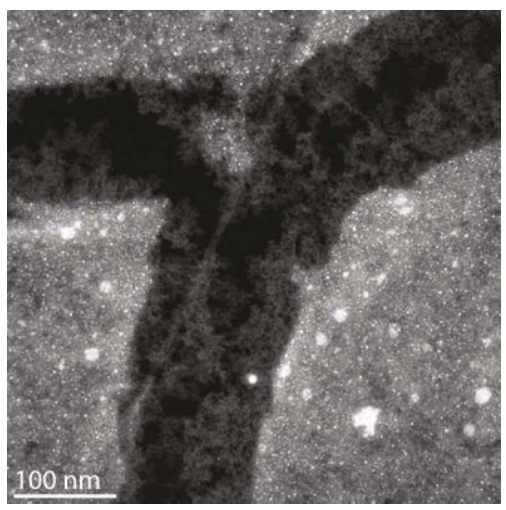

Figure 2.7. Graphene with PMMA residues (black and grey) and metal oxide particles (white) after transfer to a TEM grid.

\subsubsection{Magnetron Sputtering}

Magnetron sputtering is a Physical Vapor Deposition (PVD) technique, based on the physical ejection of atoms (sputtering) from a source (target), see Figure 2.8, instead of chemical interactions which is the basis for CVD. Atoms are ejected from the target by a plasma created by introducing argon into the evacuated

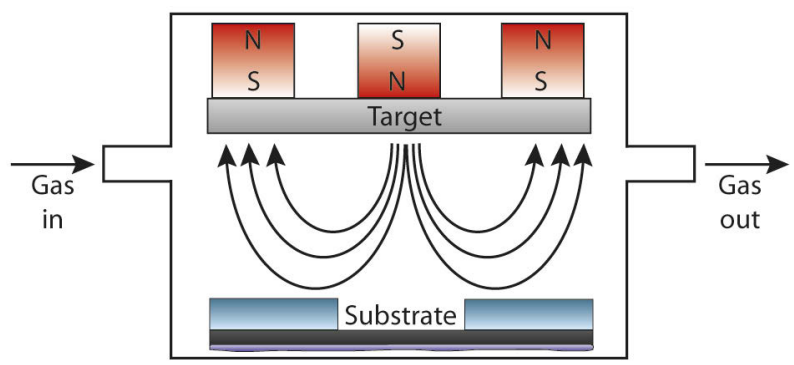

Figure 2.8. Schematic of the magnetron sputtering process. The substrate is a representation of a graphene sheet transferred to a TEM heating chip (blue) with PMMA residues (lilac) on the surface opposite to the surface facing the target. 
growth chamber. Electrons from the natural background radiation ionize the argon atoms, which are attracted to the target by a high negative voltage compared to the grounded chamber. To enhance the sputtering yield, magnets are positioned behind the target. This traps the electrons close to the target and increases argon ionization. Atoms ejected from the substrate by the argon ions travel through the chamber and are deposited on the substrate. The energy of the sputtered atoms can be controlled by many parameters, e.g. current and voltage of the target, or the chamber pressure. For most thin film depositions, an argon pressure of 3 mTorr $(\sim 0.4 \mathrm{~Pa})$ is employed. However, for the deposition of atoms onto a freestanding graphene sheet, the kinetic energy of the sputtered atoms is too high, causing the atoms to puncture the sheet. Therefore the argon pressure was increased to 30 mTorr $(\sim 4 \mathrm{~Pa})$, which increases the number of interactions between sputtered atoms and plasma. The average kinetic energy of the sputtered chromium atoms was therefore decreased enough to allow deposition onto the surface of graphene without damaging the material.

\subsection{Characterization}

The graphene sheets studied in Paper I and Paper II were imaged by Scanning Electron Microscopy (SEM) prior to deposition of metal particles and heating to confirm a successful transfer and quality of the graphene.

In a SEM an electron probe scans over a specific area of the sample and the size of the area determines the magnification. The emitted secondary electrons (see Sections 4.2.1 and 4.4.1 for further details on electron-sample interactions) are registered by a back-scatter detector, see Figure 2.9. Most detected secondary electrons originate from a small volume near the surface of a bulk sample, as the mean free path is low. The energy of the electron probe in a SEM is usually between $5-30 \mathrm{keV}$ and the lateral resolution is $\sim 10 \AA$ [95].

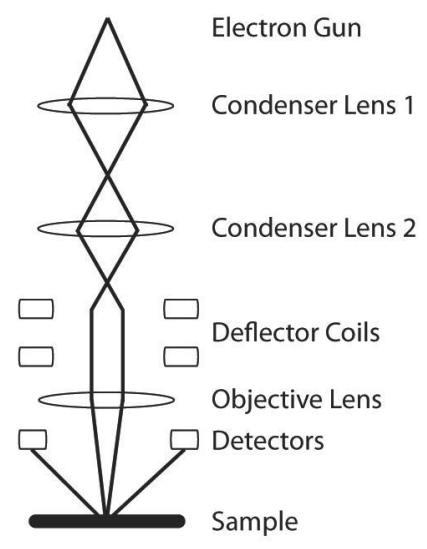

Figure 2.9. Schematic of a SEM. 


\subsection{High Temperature Annealing}

Prior to deposition of chromium atoms, graphene was placed onto chips designed for in situ TEM studies at high temperatures. DENSsolution single tilt heating chips, consisting of a $\mathrm{SiO}_{2}$ chip with a platinum wire embedded in a $\mathrm{SiN}_{x}$ window, see Figure $2.10^{6}$, were employed, together with a DENSsolution single tilt TEM heating holder. The $\mathrm{SiN}_{x}$ windows contains holes, seen as dark spots in Figure $2.10 \mathrm{c}$, enabling imaging of graphene without interference from the $\operatorname{SiN}_{x}$. The holder is stable enough to enable atomic resolution at $1300{ }^{\circ} \mathrm{C}$ [96].

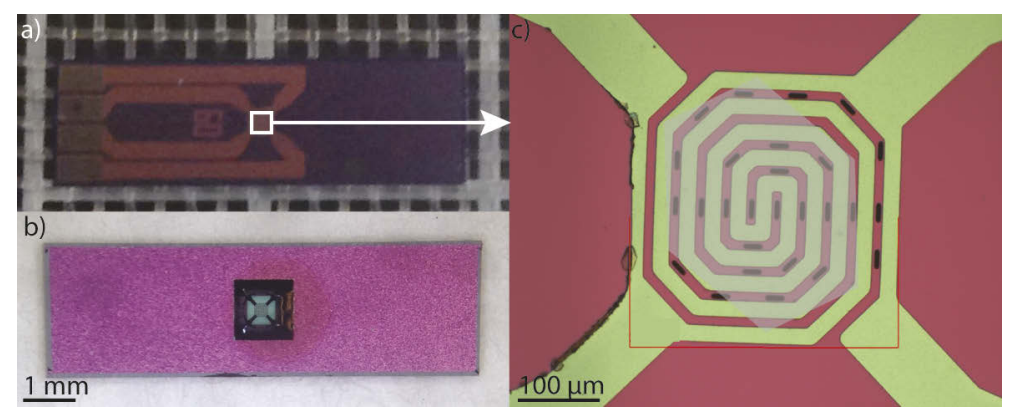

Figure 2.10. A DENSsolution single tilt heating chip used in annealing studies of graphene, showing the front side (a), the back (b) and an optical microscopy image of $\mathrm{SiN}_{x}$ window with $\mathrm{Pt}$ wires and graphene (c) prior to annealing.

During TEM imaging, the temperature was increased from room temperature up to $1300{ }^{\circ} \mathrm{C}$. This enabled observation of the PMMA decomposition, which occur in a complex chain reaction strongly dependent on type of PMMA and experimental conditions [97]. Therefore the reports on PMMA decomposition through annealing of graphene varies [90, 92, 98]. The process starts at either $160{ }^{\circ} \mathrm{C}[90,99]$ or $220{ }^{\circ} \mathrm{C}$ [98] with breakage of $\mathrm{H}-\mathrm{H}$ bonds [99]. At $270{ }^{\circ} \mathrm{C}$ the decomposition continues by breakage of unsaturated ends, creating monomers. At $360{ }^{\circ} \mathrm{C}$ these decompose into smaller molecules, such as $\mathrm{CO}, \mathrm{H}_{2}, \mathrm{CO}_{2}, \mathrm{CH}_{4} \mathrm{C}_{2} \mathrm{H}_{6}$ $[90,99]$. During this last step the decomposition products interact with defects in graphene. The interaction causes rehybridization of the $\mathrm{sp}^{2}$ bonds in graphene to $\mathrm{sp}^{3}$ bonds between graphene and the residues [90], and thus PMMA residues remain on the surface after annealing. This partially explains why graphene transferred with PMMA has rarely been reported without traces of the polymer, even after annealing at high temperatures.

PMMA also interacts with metal oxide particles on the graphene sheet during annealing. The metal oxide particles may also interact with defects and hydrocarbons on graphene at elevated temperatures [100, 101, 102, 103]. Hydrocarbons have been reported to cover the surface of graphene as graphene is exposed to air due to the reactivity of the graphene surface [104]. The preferred interaction between hydrocarbons and metal oxide particles limits the growth of metal particles

\footnotetext{
${ }^{6}$ Images a) and b) were acquired by Justinas Palisatis, Linköping University, and c) by Huy Q. Ta, Polish Academy of Sciences
} 
[100] as well as formation of uniform metallic films on graphene surfaces [103]. In Paper I, it was observed that this interaction occurs even though PMMA residues and metal oxide particles are positioned on opposite sides of the graphene sheet, and thus further limited the removal of PMMA.

Apart from interaction with the metal oxide particles, PMMA graphitize during annealing at temperatures above $500{ }^{\circ} \mathrm{C}[100,105]$, which was observed as nucleation of single graphene layers in Paper II. This reduces the removal of PMMA residues, as it would remain as additional graphene sheets. This explains the reports of clean graphene through annealing, by e.g. Xie et al. [92].

\subsubsection{Dendritic Growth}

The additional graphene sheet presented in Paper II are suggested to grow through dendritic-like growth, see Figure 2.11. Dendritic growth is a process where either a liquid is supercooled or the adatom concentration is locally supersaturated, resulting in tree-like or snowflake-like fractal patterns (dendrites) [106]. In the latter case, the adatom diffusion is limited and a spontaneous nucleation may occur. This lowers the adatom concentration near the nucleation point, which causes the adatoms to diffuse to that area and to continue the nucleation. This spontaneous nucleation occur at multiple positions, creating dendrites, shown in Figure 2.11a.

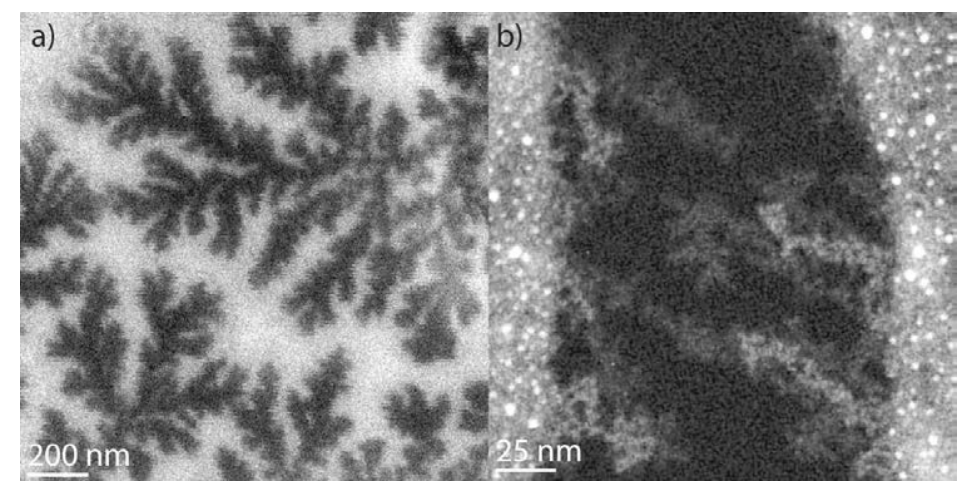

Figure 2.11. Examples of dendritic growth of carbon. SEM image of dendritic growth (a) and STEM HAADF image of dendritic-like graphene (dark grey) growth from PMMA residues (light grey) on freestanding graphene (black) (b). 


\section{CHAPTER 3}

To see a World in a Grain of Sand...

William Blake, Auguries of Innocence

Featured in the movie "Tomb Raider"

(2001)

MXene is a group of two-dimensional materials consisting of atomic layers of a transition metal and carbon. In this thesis MXene with the transition metal titanium has been studied. Unlike carbon, titanium has only two allotropes, Hexagonal Close-Packed (HCP) and Body-Centered Cubic (BCC) [107]. The element can be found in in soil, plants, and animals as well as in meteorites [108]. It is commonly used in lightweight metal alloys for applications such as space crafts, dental and orthopedic implants, as well as in jewelry. Titanium alloys with boron, nitrogen and carbon are hard, stable materials used for e.g. cutting tools. [108].

\subsection{Structure}

MXene is a nanolaminated material, consisting of a few atomic layers of two elements, see Figure 3.1. The ideal MXene composition can be described by $\mathrm{M}_{n+1} \mathrm{X}_{n}$, where $\mathrm{M}$ is a transition metal, $\mathrm{X}$ is either $\mathrm{C}$ or $\mathrm{N}$, and $n=1,2,3, \ldots$. Exfoliated MXenes exhibit surface groups and are denoted $\mathrm{M}_{n+1} \mathrm{X}_{n} \mathrm{~T}_{x}$, where $\mathrm{T}_{x}$ describe the surface groups. MXene sheets are almost always stacked, where ions and/or molecules may be positioned in between sheets without strong chemical bonding (intercalants). These intercalated MXenes are described by $\mathrm{M}_{n+1} \mathrm{X}_{n} \mathrm{~T}_{x}-\mathrm{IC}$, where IC denote the intercalants.

The first MXene was synthesized in 2011 [109] and so far 16 MXenes have been synthesized $[38,109,110,111,112,113]$ with $n=1, \ldots, 4$ while another 22 with 


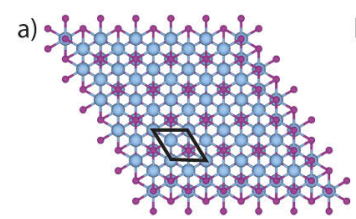

d)

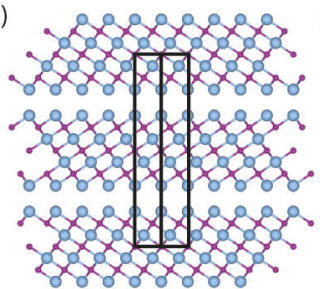

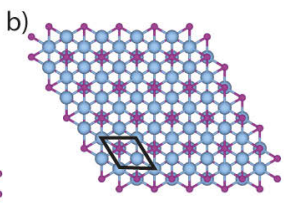

e)

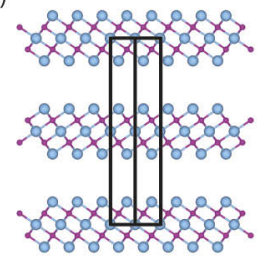

c)
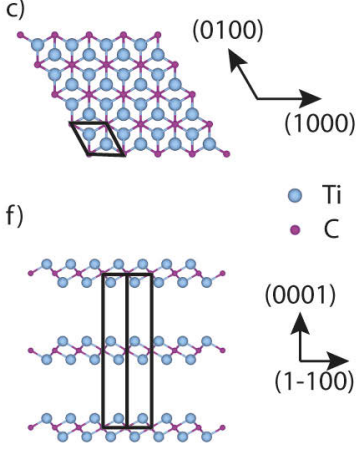

(0001)

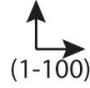

Figure 3.1. Structure model of ideal $\mathrm{Ti}_{4} \mathrm{C}_{3}$ (a,d), $\mathrm{Ti}_{3} \mathrm{C}_{2}$ (b,e) and $\mathrm{Ti}_{2} \mathrm{C}$ (c,f) with indicated unit cells.

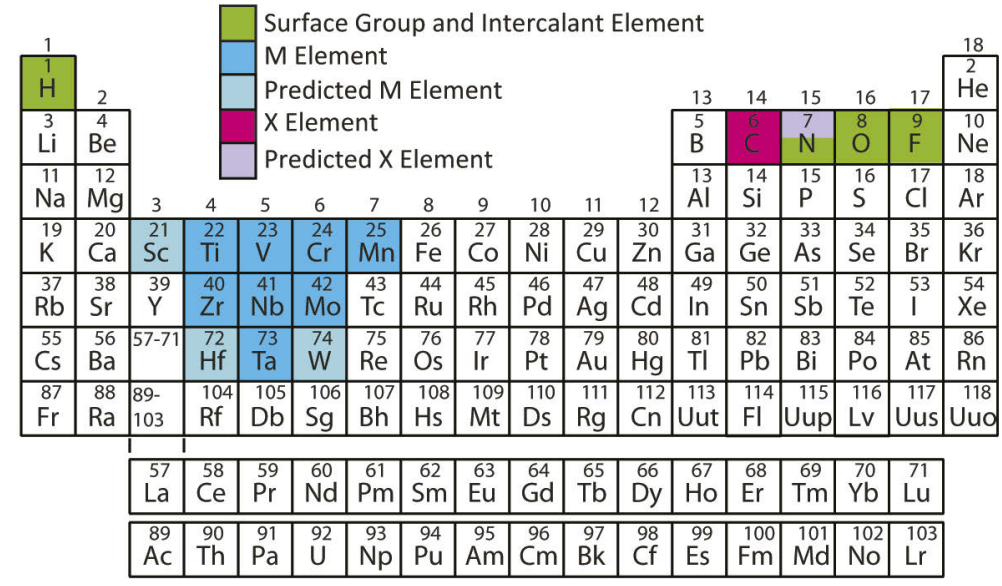

Figure 3.2. Periodic table with elements of synthesized and predicted MXenes.

$n=1, \ldots, 9$ have been predicted $[114,115,116,117,118,119]$. The elements of these are shown in Figure 3.2, of which $\mathrm{Ti}_{3} \mathrm{C}_{2} \mathrm{~T}_{x}$ is the most studied MXene to date, discussed in Papers III-V.

MXene originates from atomically laminated materials, MAX phases (see Section 3.3.1), therefore the MXene sheets are stacked and not completely separated after synthesis, appearing as either powders or thin films. The powder can be shaped by different methods, such as cold compressing which creates discs of conducting material [120] or dispersing the powders in liquid which creates a clay that can be shaped into different forms [33, 121]. The thin films are positioned on substrates and originate from an etched parent material.

In the stacked MXene, each MXene sheet appear rotated by $60^{\circ}$ with respect to the adjacent sheet [122], illustrated in Figure 3.1d-f, due to the inherent stack- 
ing of the parent material. The sheets are held together through van der Waals interaction [114, 123], which contribute to the stability of MXene [124]. Due to the low interaction between MXene sheets, the properties are independent of the number of sheets $[111,125]$. MXene sheets can be separated by different methods described in Section 3.3.2.

The structure of MXene sheets are similar to graphene, being hexagonal with space group $P 6_{3} / m m c[122,126]$, and are thus similar in plan view (compare Figure 2.2 and Figure 3.1). The unit cell of MXene $\mathrm{Ti}_{3} \mathrm{C}_{2}$ has lattice constants $a$ and $b=3.05 \AA$, while the lattice constant $c$ often describes the height of two adjacent sheets, see Figure 3.1d-f. The lattice constant $c$ is $19.86 \AA$ for ideal $\mathrm{Ti}_{3} \mathrm{C}_{2}$ MXene [122], while surface groups and intercalants expand the separation between sheets and increase the lattice constant between 0.18-9.8 $\AA$ [114, 127]. Apart from changing the separation between sheets, these surface groups and intercalants also influence the properties of MXene.

\subsection{Properties}

As MXene is a new family of materials, its properties are not yet fully understood and many investigations, both theoretical and experimental, are being performed. Most theoretical investigations have been focused on single sheets, while most experiments have investigated multiple stacked MXene sheets. Among the few experimental investigations of single sheets are Papers III-V and recently [128]. Due to the small interaction between MXene sheets, the properties of multilayer MXene is almost identical to single layers [30, 114, 125, 129]. The experimental measurements and theoretical predictions are not always consistent, as the MXene surfaces are covered by a random distribution of surface groups and intercalants which makes prediction of properties difficult. This has been observed by the few atomic resolution experiments reported so far, such as Paper III, Paper IV, Wang et al. [123], and Wang et al. [127].

The first theoretical studies of MXene assumed ideal, defect free MXenes, which predicted MXene to be metallic [114, 115, 130, 131, 132, 133]. Following studies assumed a full coverage of a single type of surface group (fluorine, oxygen, or hydroxide) and defect free MXenes, which predicted many MXenes as semiconductors with varying direct or indirect band gaps [114, 118, 124, 134, 135, 136, 137, 138]. Recently, a study assuming a random distribution of surface groups has been performed, and showed results in good correlation with measurements [123].

The measured and predicted properties of MXene are similar to those of graphene, such as stability of single sheets [136], Dirac-fermion behavior of electrons [30, 139], spin-orbit coupling [30], high transparency [111, 129], high stiffness [116] and good electrochemical performance $[132,140]$. The specific capacity for $\mathrm{Ti}_{3} \mathrm{C}_{2} \mathrm{~T}_{x}$ has been measured as $1264 \mathrm{mAh} \mathrm{g}^{-1}$ [140], which can be compared with the commonly used graphite's $350 \mathrm{mAh} \mathrm{g}^{-1}$ [40]. MXene also exhibits a conductivity comparable to multilayer graphene [16]. Also similar to graphene, the properties of MXene vary with synthesis, composition, surface groups, intercalants [28, 119, 129, $141,142]$, as well as adsorption of ions [139]. Examples are predicted magnetism in 
$\mathrm{Cr}_{2} \mathrm{C}$ and $\mathrm{Cr}_{2} \mathrm{~N}$ [114], similar to fluorine doped graphene [143], superconductivity in $\mathrm{Ti}_{2} \mathrm{CO}_{2}$ doped with $\mathrm{H}_{2}$ [139], and large Seebeck coefficient at low temperatures for $\mathrm{Ti}_{2} \mathrm{CO}_{2}, \mathrm{Hf}_{2} \mathrm{CO}_{2}, \mathrm{Zr}_{2} \mathrm{CO}_{2}, \mathrm{Sc}_{2} \mathrm{CF}, \mathrm{Sc}_{2} \mathrm{C}(\mathrm{OH})_{2}, \mathrm{Sc}_{2} \mathrm{CO}_{2}$, [114] and $\mathrm{Mo}_{2} \mathrm{CF}_{2}$ [144].

MXene's properties makes it a good candidate for anode material for Libatteries [39], commonly made of graphite which has a moderate specific lithium capacity and poor rate capability [40]. MXene is also promising as a capacitor for energy storage (super capacitors, fuel cells) [16, 121], and hybrid cells $[145,146,147]$. Further, $\mathrm{Ti}_{3} \mathrm{C}_{2} \mathrm{~T}_{x}$ is biocompatible, and is thus a candidate for electrochemical biosensors [148].

\subsection{Synthesis}

\subsubsection{MAX Phase Materials}

MXene is normally synthesized from MAX phase materials, see Figure 3.4. However, recently MXene has been synthesized from other layered materials [149]. The MAX phases are nanolaminated materials consisting of a transition metal (M), an element from group 13-16 (A) and either $\mathrm{C}$ or $\mathrm{N}(\mathrm{X})$, in the order $\mathrm{M}_{n+1} \mathrm{AX}_{n}$, where $n=1,2,3, \ldots$, see Figure 3.3. To date, there are more than 60 MAX phases synthesized. The MAX phases combine both metallic and ceramic properties, such as high thermal and electrical conductivity, elastic stiffness, and resistance to corrosion and oxidation [150]. These properties arise from a mixture of bonds, where M-A bonds are metallic and M-X bonds consist of a mixture of covalent, metallic and ionic bonds [150]. The M-X bonds are among the strongest in nature [16]. There are different methods of producing MAX phase materials, such as ball milling [109] and magnetron sputtering [129]. The $\mathrm{Ti}_{3} \mathrm{C}_{2} \mathrm{~T}_{x}$ MXene investigated in Papers III-V was synthesized from $\mathrm{Ti}_{3} \mathrm{AlC}_{2}$ powder, produced by ball milling $\mathrm{Ti}_{2} \mathrm{AlC}$ and $\mathrm{TiC}$ powders.
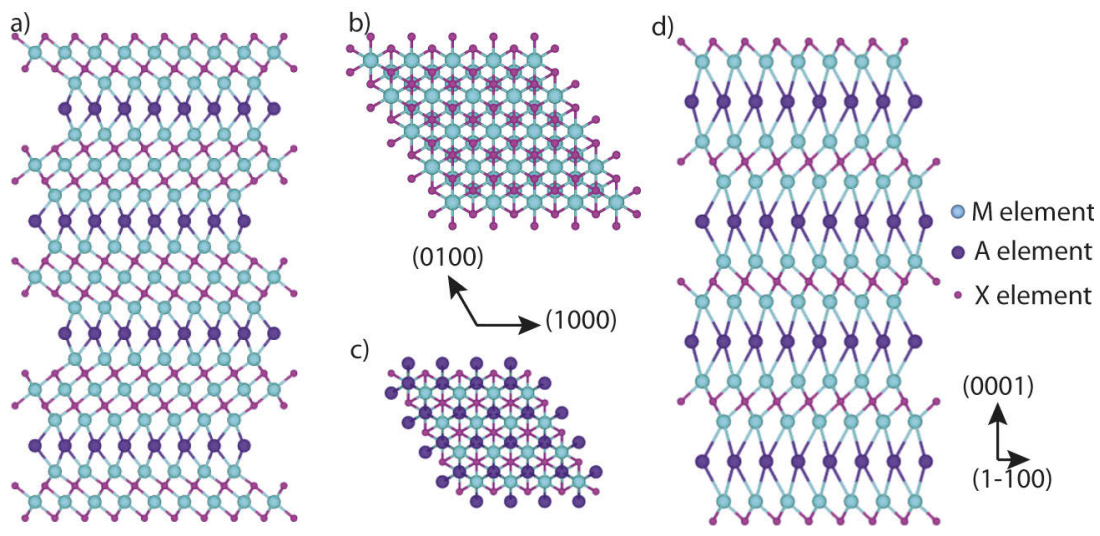

Figure 3.3. Structure of two MAX phases: $\mathrm{Ti}_{3} \mathrm{AlC}_{2}(\mathrm{a}, \mathrm{b})$ and $\mathrm{Ti}_{2} \mathrm{AlC}(\mathrm{c}, \mathrm{d})$. 


\subsubsection{Selective Etching}

MXene is synthesized from the MAX phase by etching the A element, see Figure 3.4, which replaces the metallic M-A bonds with van der Waals interaction [16] or hydrogen bonds $[123,127]$. The M-X bonds are unaffected by the process [16]. During the etching, the MX sheets are separated and are referred to as MXene $[16,122,129]$. The name MXene is derived from the name MAX phase, removing A to symbolize the removal of the A element, and -ene symbolizing the similarity to graphene [109]. The separation of the sheets greatly increase the total surface area, as the sheets are only a few $\AA$ thick [124]. The separation is aided by ions and molecules from the etchant which intercalate between the sheets during etching $[16,110,122,135]$. These molecules completely cover the surfaces without order and remain after synthesis. Oxygen, fluorine and hydroxide bonds to the MXene surfaces by hydrogen bonds [135], while other, such as $\mathrm{H}_{2} \mathrm{O}$, are loosely connected to the surface by van der Waals interaction [151]. After synthesis some MAX phase material might remain among the separated MXene sheets [111, 135, 151].

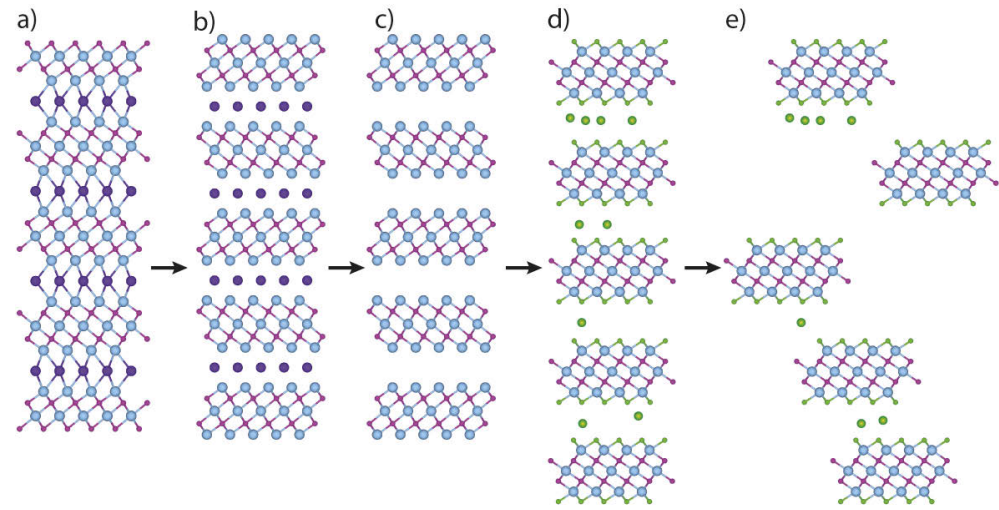

Figure 3.4. Schematic of MXene synthesis from MAX phase by etching of the Alayer (a-c), which increases separation between sheets and introduces surface groups and intercalants (d). Crushing the resulting powder results in almost complete separation between sheets $(\mathrm{e})$.

Different etchants and etchant concentrations have been employed in the synthesis of MXene, resulting in a variation of defects, crystallinity and stacking ordering of the sheets $[33,109,128,123,129,135]$. Depending on the choice of etchant, MXene can exhibit enhanced properties such as higher volumetric capacitance for lithium-based etchants [33, 152]. The variation in properties due to the various etchants are strongly correlated to changes in surface groups and intercalants, which affect the chemistry of the MXene sheets as well as the separation between sheets $[123,135]$. Etching $\mathrm{Ti}_{3} \mathrm{AlC}_{2}$ with $\mathrm{HF}$, as in Paper IV, results in $\mathrm{OH}^{-}$and $\mathrm{F}^{-}$binding to the surface of $\mathrm{Ti}_{3} \mathrm{C}_{2}$ [109]. Etching $\mathrm{Ti}_{3} \mathrm{AlC}_{2}$ with $\mathrm{NH}_{4} \mathrm{HF}_{2}$, as in Paper III and Paper $\mathbf{V}$, also causes intercalation of $\mathrm{NH}_{3}$ and $\mathrm{NH}_{4}^{+}$[152].

After etching, the sheets are not separated into individual sheets, but remain 
as multilayers [152]. The separation is increased by either sonification [109] and dispersion in ethanol [128], or dispersion in dimethyl sulfoxide (DMSO) [141, 152]. The MXene is then rinsed and dried. A large amount of $\mathrm{H}_{2} \mathrm{O}$ may still be present in between sheets [151] and thus MXene is usually dried in vacuum at $110-200{ }^{\circ} \mathrm{C}$ for $\sim 18 \mathrm{~h}$. This removes much of the $\mathrm{H}_{2} \mathrm{O}, \mathrm{NH}_{3}$, and $\mathrm{NH}_{4}^{+}[123,135]$.

In Papers III-V sonification and dispersion in ethanol was used to separate the sheets, with subsequent crushing in a mortar in Paper III and Paper V to further separate the sheets without affecting surface groups and for producing fresh fracture surfaces. This produces small flakes with varying sizes and number of sheets, see Figure 3.5b-c. The flakes were placed on holey carbon TEM grids, which are copper grids with thin sheets of amorphous carbon with holes, see Figure 3.5ab. Due to the position of flakes on the TEM grid, they exhibit random orientation. Thus some could be observed in cross section and some in plan view, compare Figure 3.5c-d. On some flakes, single or few MXene sheets were protruding from the side and could be analyzed, see Figure $3.5 \mathrm{~d}$.

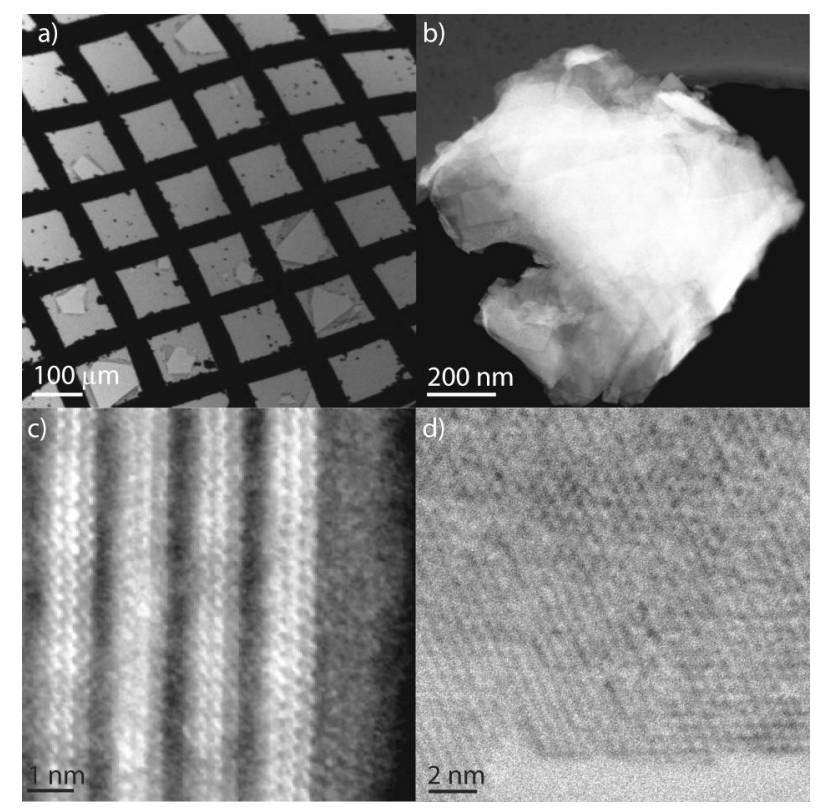

Figure 3.5. TEM of MXene at different magnifications: overview of holey carbon TEM grid with MXene particles (a), TEM image of a typical MXene flake resting on a carbon support (b), high resolution STEM HAADF image of the layering of MXene sheets shown from folded MXene where the diffuse areas are surface groups (c), and high resolution STEM HAADF image of a single MXene flake (d). 


\subsection{Characterization}

To confirm a successful etching of the MAX phase material in Paper III and Paper V, X-ray Photoelectron Spectroscopy (XPS) was performed on the resulting powder prior to TEM analysis.

XPS identifies binding energies of atoms by measuring electrons ejected from the surface of a sample by the photoelectric effect during low-energy X-ray irradiation. Measuring the kinetic energy of the emitted electrons provides information regarding composition and chemistry of the sample, as the kinetic energy $\left(E_{K}\right)$ depends on the electron bound state $\left(E_{B}\right)$, the energy of the incident X-ray $(h \nu)$ and the work function of the instrument $(\phi$, least amount of energy needed to remove an electron from the sample) through:

$$
E_{K}=h \nu-\phi-E_{B}
$$

The composition and chemistry of the sample is determined by calculating $E_{B}$, which value depends on element and exhibit small shifts depending on the how the atoms are bonded.

XPS is surface sensitive, detecting shifts in electron levels between 50-100 into a sample. However, the lateral resolution is limited, normally between $5 \mathrm{~mm}$ and $75 \mu \mathrm{m}[95]$.

\subsection{Surface Groups}

Surface groups are atoms and molecules, most commonly oxygen, fluorine and hydroxide, which are chemically bonded to the surfaces of MXene sheets and originate from the synthesis process. This can be observed in Figure 3.5c, where the diffuse areas between and on the edges of MXene sheets are surface groups. Surface groups attach to dangling bonds on the MXene surfaces [16, 122], thus increasing the thermodynamical stability of the MXene sheets [114]. Distribution of surface groups are random for these as-prepared sheets, observed in Paper III, providing a complicated surface chemistry [124], which affects stacking faults, interlayer spacing, and electronic structure [123]. As also shown in Paper III, the surface groups may also diffuse on the surface of MXene, towards the lowest energy positions, such as step edges or defects. The diffusion of oxygen on ideal MXene $\mathrm{Ti}_{2} \mathrm{C}$ and $\mathrm{Ti}_{3} \mathrm{C}_{2}$ is almost barrierless [153], which increases the difficulty in predicting the properties of MXene.

Theoretical calculations have predicted the most stable positions of surface groups on defect free MXene, although experimental observations have shown the surface groups to be randomly oriented. Different positions on $\mathrm{Ti}_{3} \mathrm{C}_{2} \mathrm{~T}_{x}$ are shown in Figure 3.7, here following the notation in [153]. The most stable position is directly on top of the middle titanium atom (fcc, also referred to as Configuration I or Configuration A), the second most stable is on top of a carbon atom (hcp, also referred to as Configuration II or Configuration B) $[118,119,132,136,151,153,154,155]$, the second least stable is between the middle titanium and a carbon (bridge) and the least stable is on top of a outer 

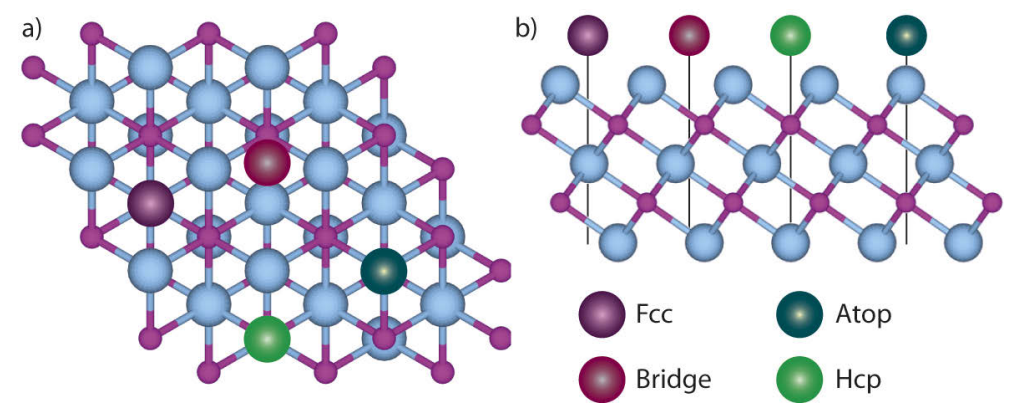

Figure 3.6. Stable positions of surface groups on $\mathrm{Ti}_{3} \mathrm{C}_{2}$, shown in top view (a) and side view (b): atop, on top titanium atom, bridge, between two middle titanium and upper carbon, fcc, on top of middle titanium, and hcp, on top of carbon, as given by [153]. Note that the size of the surface groups are enlarged to increase visibility.

titanium (atop) [116, 136, 153, 155, 156]. The fcc position is more stable due to repulsion between carbon and surface groups [115, 118, 132]. In the theoretical calculations assuming full, uniform coverage of surface groups, the surface groups are either positioned at the fcc or hcp position on both sides, or one side has hcp and the other fcc (Configuration III). This third configuration has less stability than the fcc and hcp [118, 119, 132, 154, 155].

Results from experimental studies on the position of surface groups on MXene show that the positions are random and there is no long range order [123, 127, 135]. However, hcp appear to be the most common position on $\mathrm{Ti}_{3} \mathrm{C}_{2} \mathrm{~T}_{x}$ for oxygen and fluorine $[122,124,127]$, except when using $50 \% \mathrm{HF}$ solution, then hcp and fcc are equally common [124]. Hydroxide is the most common surface group on $\mathrm{Ti}_{3} \mathrm{C}_{2} \mathrm{~T}_{x}$ $[124,155]$ and has no preferred position on the Mxene surface [122]. However, surface groups may cluster, as shown in Paper III and reported in [151]. In Paper III it was also noted that surface groups appear not to cover the entire surface of the MXene sheet. The variations of surface group positions may also be due to the replacement of fluorine by hydroxide [157, 146, 158] or by oxygen during storage in water [124].

Depending on surface group and position on the lattice, the distance to the MXene surface varies, and thus also the separation between sheets [118]. The average distance between titanium atoms and surface groups is $\sim 3.5 \AA[115,124,132]$. The surface groups on two facing sheets interact via van der Waals interaction [114], which depend on the amount of surface groups on each surface [123]. MXenes completely covered by oxygen is predicted to have a smaller separation of sheets than fluorine or hydroxide covered MXene [119], and measurements have shown that fluorine is closer to the MXene surface than hydroxide [151]. The interaction between surface groups are affected by the stacking sequence. If the sheets are stacked so that different surface groups are facing each other, they are attracted and the distance between the sheets is small. However, if the sheets are stacked so that the same surface groups are facing each other, or if there is a high concentration of a single surface group, the surface groups repel each other, increasing 
the distance between sheets [123].

\subsubsection{Effect on Properties}

The M-X bonds in $\mathrm{Ti}_{3} \mathrm{C}_{2} \mathrm{~T}_{x}$ MXene occur due to hybridization of titanium d states and carbon $\mathrm{p}$ states, which causes its unique properties. In ideal $\mathrm{Ti}_{3} \mathrm{C}_{2}$, titanium $\mathrm{d}$ orbitals lie close to the Fermi level $[114,115,155]$, and are hybridized by the carbon p orbitals $[114,115,155]$. These orbitals are affected by the type, position and coverage of surface groups $[114,136,155]$. The surface groups attract electrons from the transition metal, one electron for $\mathrm{F}^{-}$or $\mathrm{OH}^{-}$and two for $\mathrm{O}^{2+}$ [114], which causes a reduction of the titanium d orbitals by the fluorine $\mathrm{p}$ or oxygen $\mathrm{p}$ orbitals, shifting the Fermi level $[114,115,153,155]$. The shift is also affected by changing positions of surface groups, causing a change of the $\mathrm{C}-\mathrm{O}$ bond lengths [139]. This can be observed as a change of surface plasmons [125] and shift of core electrons, which can be observed by Electron Energy Loss Spectroscopy (EELS) (see Section 4.4.2). In Paper III, this was observed to affect the local electronic structure. In Paper V, it was observed that surface plasmons on single MXene sheets are sensitive to number of sheets and surface groups.

As the electronic structure changes, the properties of MXene change. This has been studied both experimentally and theoretically. All studies of ideal MXenes predict metallic properties, with similarities between MXenes depending on group of M element [16, 109, 114, 124]. The results for MXene with surface groups varies depending on whether the study is experimental or theoretical, and assumptions used in the theoretical studies. MXenes covered with uniform surface groups are predicted to be either semiconducting with either direct or indirect band gaps $[16,109,114,124,153]$ or metallic $[110,118]$. The band gap of oxygen covered MXene is also predicted to depend on position of surface groups [132]. Other properties are affected as well, a few examples are given here; theoretical studies have predicted that ideal MXenes have higher cyclic rates than oxygen or hydroxide covered MXenes [159], while measurement of storage capacity showed the highest storage capacity for MXene's mainly covered by oxygen [124]. Oxygen covered MXenes are also predicted to have higher mechanical strength than fluorine or hydroxide covered MXenes [119]. In experiments, the optical transmittance has been observed to change up to $40 \%$ depending on surface group [155].

Ideal $\mathrm{Ti}_{3} \mathrm{C}_{2}$ is predicted as metallic, while $\mathrm{Ti}_{3} \mathrm{C}_{2} \mathrm{~F}_{2}$ semiconducting [132], $\mathrm{Ti}_{3} \mathrm{C}_{2}(\mathrm{OH})_{2}$ either metallic [118] or semiconducting [132], and $\mathrm{Ti}_{2} \mathrm{CO}_{2}$ either semiconducting [114, 115] or insulating [139]. However, experiments show that $\mathrm{Ti}_{3} \mathrm{C}_{2} \mathrm{~T}_{x}$ is metallic even with surface groups [129] and that single $\mathrm{Ti}_{3} \mathrm{C}_{2} \mathrm{~T}_{x}$ sheets are metallic with n-type conductivity [128].

\subsection{Intercalants}

Intercalants are molecules not bonded directly to the surface of MXene sheets, but are positioned in between sheets, see Figure 3.7. Entire surfaces or parts of surfaces may be covered, but the intercalants are not localized at specific positions as they easily diffuse [127] and have been observed near defects [122]. Interaction 
between intercalants and surface groups may cause a change of surface group position [127, 160] and increase the distance between sheets depending on type of intercalant $[16,122,127,141,142,145]$. Some intercalants may also shift the relative positions of sheets, increasing sheet interaction [127] as well as change MXenes electronic structure [122, 127].

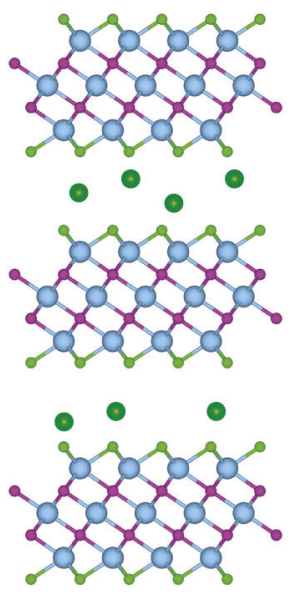

Figure 3.7. Schematic of intercalated $\mathrm{Ti}_{3} \mathrm{C}_{2} \mathrm{~T}_{x}-\mathrm{OH}$ MXene (intercalants are shown in dark green).

Molecules that are intercalated during synthesis are $\mathrm{H}_{2} \mathrm{O}$, fluorine containing molecules from $\mathrm{HF}$ and $\mathrm{NH}_{4} \mathrm{HF}_{2}$ [142], ammonia $\left(\mathrm{NH}_{3}\right)$ and ammonium $\left(\mathrm{NH}_{4}^{+}\right)$ from $\mathrm{NH}_{4} \mathrm{HF}_{2}$ [129]. $\mathrm{H}_{2} \mathrm{O}$ is the most common intercalant and is bonded to hydroxide by hydrogen bonds [151], which limits van der Waals interaction between sheets $[124,155]$. Other molecules have consciously been intercalated between MXene sheets in order to change properties or increase separation of sheets. Examples are $\mathrm{K}^{+}, \mathrm{Na}^{+}[122,127], \mathrm{Mg}^{2+}, \mathrm{Al}^{3+}[159]$, hydraxine, urea [141] and $\mathrm{Li}^{+}$ [145]. Intercalating aluminium [127] or lithium [145] increases the storage capacity of $\mathrm{Ti}_{3} \mathrm{C}_{2} \mathrm{~T}_{x}$. Lithium absorption depends on both type and coverage of surface groups [124, 132].

\subsubsection{Effect on Properties}

Adsorption of ions on the MXene surface also changes the properties. Adding hydrogen, lithium, or sodium onto MXene covered with surface groups is predicted to make it metallic [139]. The increased separation affect the properties of MXene, such as decreasing the transmittance of visible light by 10-30\% [129]. 


\section{CHAPTER 4}

\section{Aberration-Corrected Transmission Electron Microscopy}

That's no moon.

Ben Kenobi

Star Wars: A New Hope (1977)

As a material's properties originates from its atomic structure, it is imperative to study this in order to understand the properties. TEM is a powerful tool as it allows direct imaging in combination with spectroscopy of the crystal lattice. The latest generation of TEMs can resolve single atoms, enabling studies of how individual atoms or molecules affect material properties, such as surface groups on two-dimensional materials.

The distance between atoms in a lattice is in the order of a few $\AA$. Thus human eyes and standard Visible Light Microscopes (VLM) cannot be employed to study crystal structures or atoms, as the smallest distance which can be separated (point resolution) by a human eye is in the order of $0.1 \mathrm{~mm}$ at a distance of $40 \mathrm{~cm}$ [161] and by a standard VLM $\sim 5000 \AA$ [162]. The point resolution for an optical system $\left(d_{0}\right)$ is described in a classical form by the Rayleigh criterion [163];

$$
d_{0}=\frac{0.61 \lambda}{n \sin \alpha}
$$

where $\lambda$ is the wavelength of the illumination, $\alpha$ the incident angle, and $n$ the refractive index of the medium. Decreasing the wavelength or improving the microscope optics increases the point resolution. However, as the human eye can only see wavelengths between $3800-7500 \AA$, there is a limit to how much the wavelength can be decreased. Therefore, microscopes employing other wavelengths are equipped with detectors which can observe wavelengths far beyond what the human eye can detect. 
Improving the microscope optics allowed Eric Betzig, Stefan W. Hell, and William E. Moerner to achieve point resolutions $(\leq 500 \AA)$ far beyond the Rayleigh criterion with visible light microscopes, so called 'superresolution microscopes'. For their discoveries they were awarded the 2014 Nobel Prize in Chemistry [164].

In TEM, electrons are employed instead of light as illumination. Electrons can be utilized as they can be viewed as both particles and waves, where the wavelength is determined by their kinetic energy. Electrons with an energy of $60 \mathrm{keV}$, used in low-voltage TEM, have a wavelength in the order of $0.05 \AA$ (10 $0^{5}$ times smaller than the wavelength of visible light), while electrons with an energy of $200 \mathrm{keV}$, used in standard TEM, have a wavelength in the order of $0.03 \AA$. The electrons are transmitted through a thin sample, see Figure 4.1, and subsequently detected by various detectors, described in Sections 4.2.2 and 4.2.3. As electrons interact easily with air, the TEM is held at a ultra high vacuum to ensure interaction with only the sample, reducing $n$ to 1 . Thus in a modern TEM, the short wavelength of electrons, the small scattering angles of the electrons, and the refractive index of vacuum provides an optimum resolution of $\sim 1 \AA$ for $60 \mathrm{keV}$ electrons and $\sim 0.5 \AA$ for $200 \mathrm{keV}$ electrons.

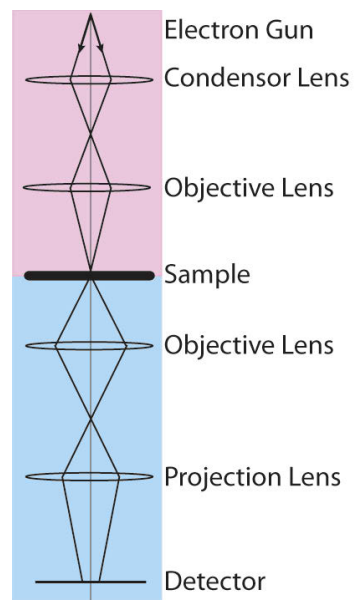

Figure 4.1. Schematic of the basic principle of a (S)TEM. Electrons are accelerated by an electron source, focused onto the sample by the objective lens and the transmitted electrons are detected. Lenses positioned along the optical axis control the illumination onto sample and detectors.

The point resolution is however limited by the inherent imperfections of the electromagnetic lenses, which create magnetic fields that manipulate the electron paths through the TEM. These imperfections (aberrations) increase the point resolution to a few $\AA$ for electrons accelerated by a voltage of $60 \mathrm{kV}$. At this point resolution not even the crystal lattice can be resolved, and thus standard TEMs are used with 200-300 kV acceleration voltage. Higher energies up to $1.3 \mathrm{MeV}$ have been employed, reaching a resolution of $0.6 \AA$ [165].

During the last decade new TEM models have become available which mini- 
mizes the most common aberrations, thus enabling imaging at $60 \mathrm{keV}$ with a point resolution of $\sim 1 \AA[166]$.

\subsection{The Transmission Electron Microscope}

A TEM analyzes specimens in two fundamental modes: Conventional Transmission Electron Microscopy (CTEM), in which a broad, parallel beam illuminates the sample, and STEM, where a small converging probe scans over the sample, see Figure 4.2. In an aberration-corrected microscope the probe size can be below $1 \AA$. In STEM and CTEM, image contrast and intensity variations provide information regarding the microstructure of a sample. The intensity is the amount of electrons per image pixel while contrast is the intensity differences between pixels in the image.

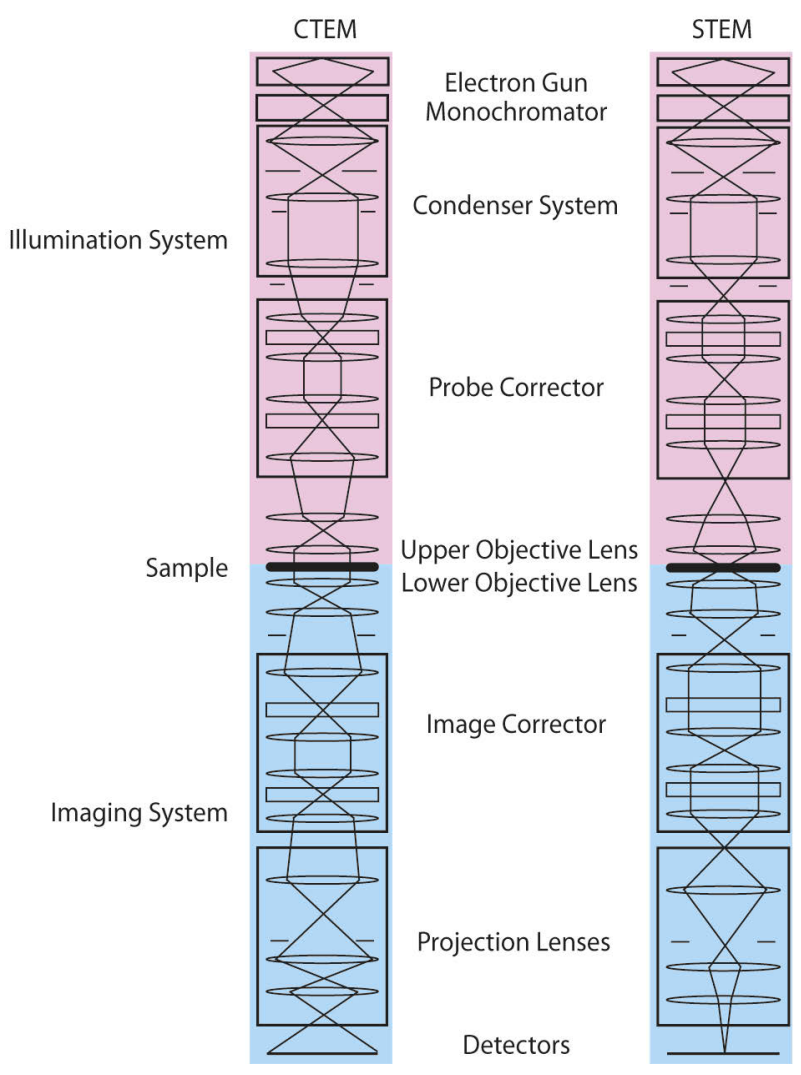

Figure 4.2. Schematic of a TEM column of an aberration-corrected microscope, showing the electron path from electron source to detector for both CTEM (left) and STEM (right) modes. Note the differences on the sample and detector. 
Some TEMs can be employed in both CTEM and STEM mode, while other microscopes are dedicated to one mode. Two microscopes were used for the Papers in this thesis, the Linköping monochromated, double-corrected FEI Titan ${ }^{3}$ 'Arwen' which employs both modes, and the SuperSTEM facility's monochromated, probe corrected Nion UltraSTEM ${ }^{\mathrm{TM}}$ 100MC 'HERMES', which is a dedicated STEM (but can be operated in CTEM mode). The Titan ${ }^{3}$ was employed for Papers IIV and the UltraSTEM ${ }^{\mathrm{TM}}$ for Paper $\mathbf{V}$ due to its high energy resolution, which is $\sim 4$ times higher than the Titan ${ }^{3}$.

The TEM column can be divided into two parts, the Illumination System which control the electron energy and spread onto the sample and the Imaging System which projects the transmitted electrons onto detectors, see Figure 4.2. Between the two parts the sample stage is positioned, in which the sample is placed. The sample stage controls the position and rotation of the sample between the upper and lower objective lenses. The upper objective lens shapes the electrons to either a beam or a probe onto the sample, and the lower objective lens forms the image which is transmitted through the imaging system. As it is the lens closest to the sample, aberrations in the objective lens are the most limiting factors on point resolution in CTEM images.

\subsubsection{Illumination System}

\section{Electron Gun and Monochromator}

The function of the electron gun is to extract and accelerate electrons to a specific energy. For standard TEMs the most common energies are 200 or $300 \mathrm{keV}$, which provides a point resolution $\sim 2 \AA$. In aberration-corrected TEMs the energy can be reduced to $20-60 \mathrm{keV}$ and still maintain similar or better resolution $[166,167]$. In this thesis, $60 \mathrm{keV}$ has been employed since it is below the damage threshold for two-dimensional materials, see Section 4.5.

Aberration-corrected TEMs have electron sources with high current, small angular spread of electrons (high brightness) and small spread of electron energy (energy resolution). These TEMs employ Field Emission Guns (FEGs), which consists of a sharp tip of $\mathrm{W}$ with a diameter of less than $1000 \AA$ [163]. A high voltage is applied on the tip which creates an electric field that causes electrons to tunnel from the tip into an accelerator. The tip is sensitive to contamination, and in the two TEMs employed for this thesis two different methods are employed to limit the contamination. In the $\operatorname{Titan}^{3}$, the tip is continuously heated (X-FEG), while the UltraSTEM ${ }^{\mathrm{TM}}$ tip is held at ultrahigh vacuum (cold-FEG). Heating the tip prohibits contaminants from adsorbing on the surface while a ultrahigh vacuum severely limits the amount of contaminants near the tip.

The energy resolution of a X-FEG is $\sim 1.1 \mathrm{eV}$ at an acceleration voltage of $60 \mathrm{kV}$. For high resolution spectroscopy, as well as atomic resolution imaging, the energy resolution needs to be improved. This is enabled by the monochromator positioned before the accelerator in the $\operatorname{Titan}^{3}$, see Section 4.3.5, and in the condenser system in the UltraSTEM ${ }^{\mathrm{TM}}$ [168]. The energy resolution can be filtered to $70 \mathrm{meV}$ in the $\operatorname{Titan}^{3}$ at $60 \mathrm{kV}$, but is more commonly employed at $\sim 180 \mathrm{meV}$ to balance the beam current, and to $\sim 17 \mathrm{meV}$ at $60 \mathrm{kV}$ in the $\operatorname{UltraSTEM}^{\mathrm{TM}}$. 


\section{Condenser System}

The condenser system consists of a series of lenses and apertures which change the beam current and intensity spread of the electrons. It also increases the spatial coherency of the electrons, i.e. minimizing the variation of the spatial phase component of the electrons. A high spatial coherence is important for atomic resolution STEM imaging, which is described in Section 4.2.2.

\section{Probe Corrector}

The STEM probe is sensitive to aberrations in the illumination system which affect the point resolution of the images, and thus both the $\mathrm{Titan}^{3}$ and the UltraSTEM $^{\mathrm{TM}}$ are fitted with aberration-correctors (probe correctors) positioned inside the condenser system. The function of the probe corrector is described in Section 4.3.5.

\subsubsection{Imaging System}

For a dedicated STEM the imaging system consist of a series of detectors, while for a TEM with both CTEM and STEM capabilities, the imaging system also contains a series of lenses (projection lenses) and apertures which select a part of the image for analysis. The projection system control the magnification of the CTEM image and the aberration-corrector for CTEM imaging (image corrector) is placed there. Detectors for STEM imaging are described in Section 4.2.2 and for CTEM imaging in Section 4.2.3.

\subsection{Main TEM Imaging Techniques}

For very thin materials (such as two-dimensional materials) most electrons are transmitted through the sample without interaction, but electrons that do interact are scattered differently depending on the interaction, see Figure 4.3 which shows the most common electron-sample interactions. Electrons can either interact with the sample with negligible energy loss (elastic scattering) or with energy loss (inelastic scattering). The various interactions together with the different TEM modes provide a large diversity of information regarding the sample.

\subsubsection{Elastical Scattering}

The contrast in TEM images from thin samples originate mainly from elastic scattering, which occur due to electron interaction with atoms, see Figure 4.18 where the electron is viewed as a particle. As the sample thickness increases, the inelastic scattering becomes the more dominant interaction.

\subsubsection{STEM Imaging}

As the STEM probe scans over the sample, the STEM detectors register the amount of scattered or transmitted electrons at each probe position for a selected 


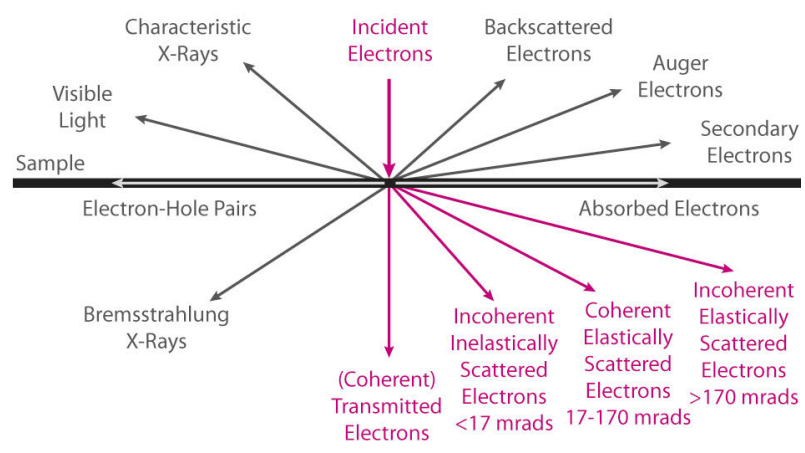

Figure 4.3. The most common interactions between electrons and sample (scattering angles from [163]). The pink text shows the interactions used for imaging in TEM.

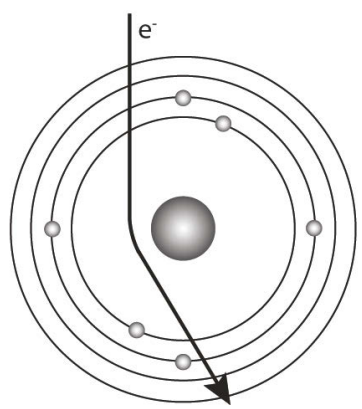

Figure 4.4. Elastic scattering of an incident electron with an atom.

scattering angle, see Figure 4.5a. The Bright Field (BF) detector registers unscattered electrons between 0-10 mrad, see Figure 4.5c, while the other detectors register scattered electrons. As the number of scattered electrons increase with increased atomic number $(Z)$ by $Z^{1+\frac{2}{3}}[163]$, the BF and Annular Dark Field (ADF) (11-40 mrad) are better for detecting low mass elements while the Medium-Angle Annular Dark Field (MAADF) (40-65 mrad) and HAADF (>65 mrad) detects all elements while displaying little contrast for very low mass elements (e.g. hydrogen, helium, lithium). The detectors (except for the BF) are annular with different radii and can thus be employed simultaneously. Of these detectors, HAADF is the most commonly used in STEM mode as the image contrast is directly related to variations in mass or thickness. STEM HAADF images are therefore called Z-contrast images, exemplified in Figure 4.5b.

The scattering angle incident on the detectors can be controlled by changing the virtual distance between detector and sample (camera length), see Figure 4.5a. As the distance between sample and detectors is fixed in a TEM, the strength of the projection lenses are altered to change camera length. 


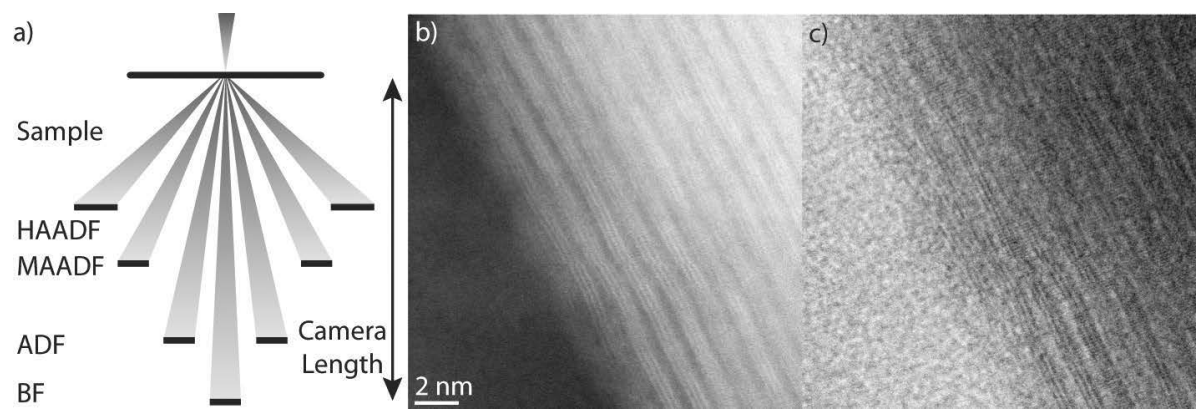

Figure 4.5. The various STEM detectors (a) and examples of a STEM HAADF image (b) and a STEM BF image (c) of MXene $\mathrm{Ti}_{3} \mathrm{C}_{2} \mathrm{~T}_{x}$.

\subsubsection{CTEM Imaging}

As the beam in CTEM imaging illuminate an area of the sample, the transmitted beam forms an image which is recorded on a CCD camera. Depending on magnification, different types of electron-matter interactions dominate the contrast formation, see examples in Figure 4.6. The electrons are viewed as waves and not particles in CTEM imaging, as the different contrast mechanisms are easily explained for the interaction between waves. In CTEM imaging either the transmitted, unscattered beam can be detected ( $B F$ imaging) or a specific scattered beam can be detected (Dark Field (DF) imaging). The resolution of a CTEM image is additionally affected by the Point Spread Function (PSF), which describes how the CCD transforms a point in the sample to a disc in the image.
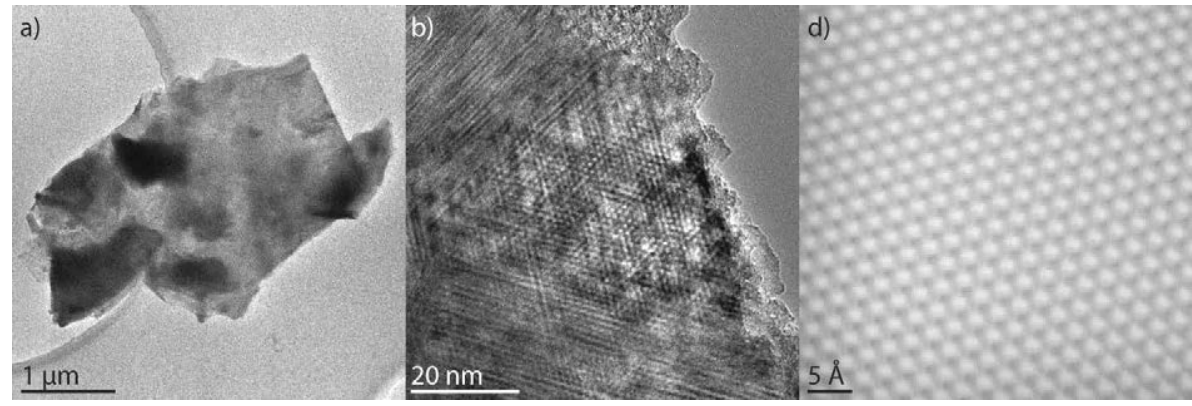

Figure 4.6. Different contrast mechanisms in CTEM: thickness contrast of MXene flake on carbon support (a), BF diffraction contrast image of MXene (b), and phase contrast image of graphene (c) where the dark dots correspond to carbon atoms.

\subsubsection{Contrast Formation}

During interaction, the phase and amplitude of the electrons change and this can be registered as contrast and intensity variations in the STEM and CTEM images. 
The interaction depends on the thickness, atomic number and crystal structure of the sample. Variations in mass or thickness (mass-thickness contrast) or diffraction (diffraction contrast) causes changes in the amplitude. These are the dominant contrast mechanism at low and medium magnifications.

During transmission through a thin sample, electrons pass through the atomic columns or in between atomic columns. As the electrons pass through the atomic columns, they interact with the atoms which causes a phase shift while the electrons passing between columns are not phase shifted. After exiting the sample, the electrons interact, appearing as either constructive or destructive interference depending on the phase variations. The phase shifts of the electrons due to this channeling are highly affected by the atomic structure and are visible at high magnifications. Therefore, it is important to reduce any phase shifts originating from sources other than the sample itself, such as lens aberrations, which distort the final image and reduce the point resolution. If the sample is thin, $<50 \AA$, the amplitude of the electron wave can be assumed constant and only the phase shift is visible in the final image.

As phase shifts are sensitive to the atomic columns, misalignment's of two or more two-dimensional sheets perpendicular to the beam are visible as a pattern which change with the misalignment (moiré patterns) (see below) [163].

\section{Mass-thickness contrast}

In thick and dense materials the electrons are scattered more than in thin and low-density materials, decreasing the amount of electrons transmitted through the sample. This is observed as a decrease of the intensity of the CTEM and STEM BF images, as in Figure 4.5b and Figure 4.6a, where the thicker areas are darker.

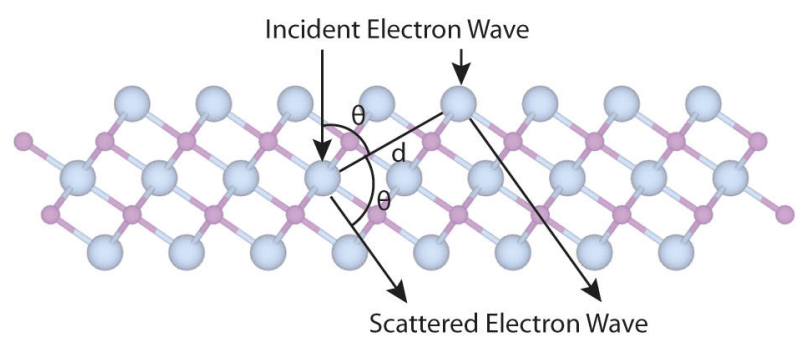

Figure 4.7. Bragg diffraction of electrons by a MXene lattice. The incident angle and the scattering angle is the same.

\section{Diffraction contrast}

Electrons can be reflected by variations in electron density in the crystal lattice (Bragg diffraction), which causes interference patterns (diffraction patterns), with constructive and destructive interference which can be seen as bright or dark lines or dots, see Figure 4.7. The diffraction patterns depend on the structure, orientation, lattice constants and domain sizes of crystals in the sample. Observing 
a CTEM image from a specific spot in the diffraction pattern gives information regarding the crystal from which that spot has originated. BF imaging creates an image including the unscattered beam while DF imaging creates an image from a diffracted beam. An example of a CTEM BF image is shown in Figure 4.6b, and influence the contrast in Figure 4.6a.

\section{Moiré pattern}

If two or more stacked two-dimensional sheets are rotated perpendicular to the electron beam, an interference pattern is visible in the CTEM or STEM phase contrast image, see Figure 4.8. A large rotation causes a pattern of small circles while a small rotation causes large circles. The rotation can be determined by creating Fast Fourier Transforms (FFTs) of the images and measuring rotation of the patterns [166].
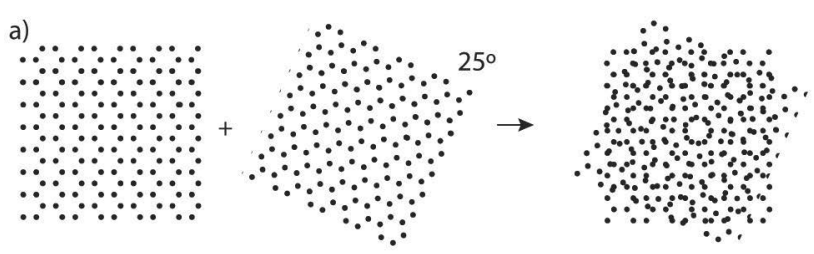

b)
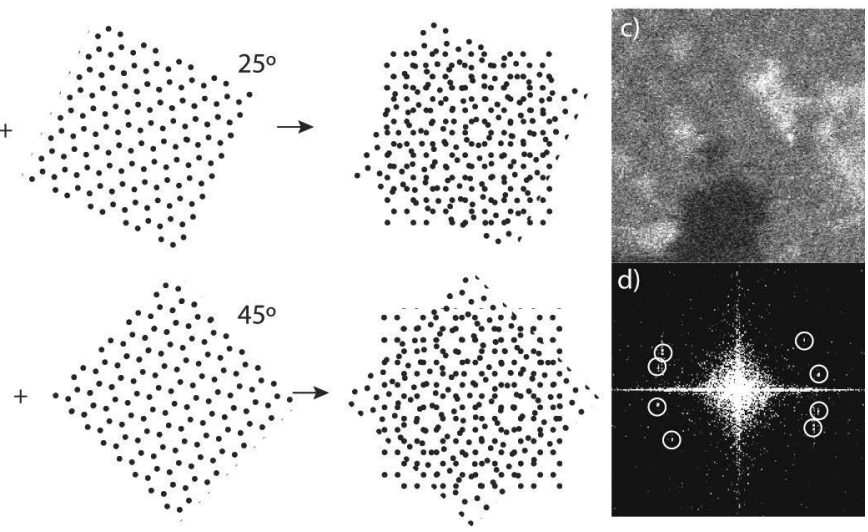

Figure 4.8. Moiré pattern of two rotated two-dimensional sheets. Schematic with $25^{\circ}$ rotation (a) and $45^{\circ}$ rotation (b), a STEM HAADF image of bilayer graphene (c), and FFT pattern from (c) shown in (d) with indication of signals from the graphene layers.

\section{Contrast Formation at Atomic Resolution}

The contrast of a high resolution image depends on phase changes due to the sample and the electromagnetic lenses, especially the objective lens.

Depending on how the sample is oriented in an image, the electrons interact differently with the atomic columns, creating variations in the resulting high resolution image. This was investigated for two types of MXenes $\left(\mathrm{Ti}_{3} \mathrm{C}_{2}\right.$ and $\left.\mathrm{Ti}_{2} \mathrm{C}\right)$ in Paper IV, where the contrast of the $\mathrm{Ti}_{3} \mathrm{C}_{2}$ appear similar to multilayer graphene and $\mathrm{Ti}_{2} \mathrm{C}$ as single layer graphene due to the increased scattering by titanium compared to carbon.

How the microscope affects the image contrast is described by the Contrast Transfer Function (CTF), which is expressed as the contrast dependence on reciprocal lattice spacing for a given objective lens defocus, see Figure 4.9. When 
the CTF value is positive, the atomic columns appear bright, and when the CTF value is negative, the atomic columns appear dark. The CTF value of vacuum is 0 as there is no contrast variation due to no electron-sample interaction. Lowering the acceleration voltage increases contrast due to increased electron-sample interaction, and thus imaging at $60 \mathrm{kV}$ results in a higher contrast than imaging at $300 \mathrm{kV}[166]$.

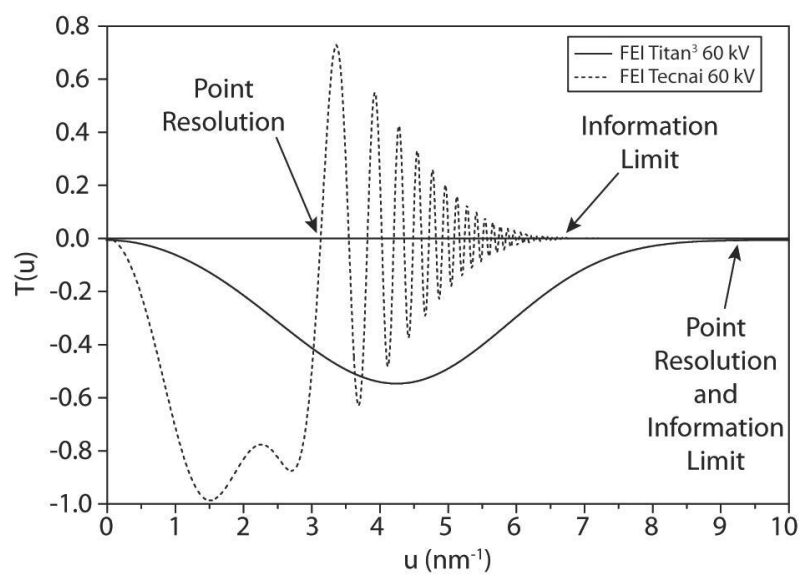

Figure 4.9. Contrast values in the CTF $(T(u))$ as a function of lattice spacing $(u)$ of a standard microscope (FEI Tecnai) and the aberration-corrected Titan ${ }^{3}$ in CTEM mode. Point resolutions and information limits of the two microscopes are indicated.

The point resolution is defined as the first crossover, while the limit after which there is no contrast at all is called the information limit. For a standard TEM the point resolution is given by

$$
r_{p}=\sqrt{\frac{3}{4}} C_{3}^{\frac{1}{4}} \lambda^{\frac{3}{4}}
$$

where $C_{3}$ is the spherical aberration, see Section 4.3.2, and $\lambda$ is the electron wavelength, while the information limit is given by

$$
r_{i} \simeq C_{C} \sqrt{\left(\frac{\Delta V}{V}\right)^{2}+\left(\frac{\Delta I}{I}\right)^{2}+\left(\frac{\Delta E}{V}\right)^{2}}
$$

where $C_{C}$ is the chromatic aberration, see Section 4.3.1, $V$ is the acceleration voltage, $I$ is the objective lens current and $E$ is the electron energy. For an aberration-corrected TEM the point resolution and the information limit is at the same position.

The CTF is often expressed as

$$
T(\vec{u})=A(\vec{u}) E(\vec{u}) 2 \sin \chi(\vec{u})
$$

where $\vec{u}$ is the reciprocal lattice spacing, $A(\vec{u})$ is the aperture function which describe the effect of apertures, $E(\vec{u})$ is the envelope function which describes the 
effect of the coherency and energy resolution, and the aberration function, $\chi(\vec{u})$, which describe the effect of lens aberrations.

\subsection{Aberrations in TEM}

Electromagnetic lenses act on electrons by the Lorentz force,

$$
\vec{F}=-e(\vec{E}+\vec{v} \times \vec{B})
$$

where $e$ is the electron charge, $\vec{v}$ the electron velocity, $\vec{E}$ the electric field of the lens, and $\vec{B}$ the magnetic field of the lens. The Lorentz force causes the electrons to deviate from their path and the deviation depends on the distance between electrons and the optical axis, the energy of the electrons, and the incident angle between electrons and the optical axis. Electromagnetic lenses are always convergent and aberrations affect the path of electrons through the lens. Aberrations are usually described as how the surface of the electron wave deviates from a perfect Gaussian surface, and cause points in the sample to appear as discs in the image.

As the lens aberrations are complex, they are usually written as a Taylor series (here in polar coordinates):

$$
\chi(\theta, \phi)=C_{0,1}+\sum_{n, m} \frac{\theta^{n+1}}{n+1} C_{n, m}
$$

where $C_{0,1}$ is the phase shift due to interactions with the sample, $C_{1,0}$ is the phase shift due to defocus of the lenses and the higher order terms are due to variations in how the electrons enter the lens [169]. There are many different notations of the aberration coefficients, here the Krivanek notation [170] is used. Further on the Haider notation [171] will be used as well as these two notations are equally common. The effect of the aberration coefficients on the phase shift decrease with higher order. Here some of the most resolution limiting aberrations are described.

\subsubsection{Chromatic aberration $\left(C_{c}\right)$}

Variations in electron energy, from both the electron gun and energy losses due to interaction with the sample, causes electrons to focus at different positions, see Figure 4.10. This is known as chromatic aberration and affect the envelope function, $E(\vec{u})$, in the CTF.

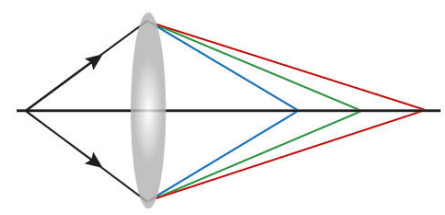

Figure 4.10. Chromatic aberration causes electrons to focus at different positions depending on their energy. 


\subsubsection{Spherical Aberration $\left(C_{3,0}, C_{3}\right.$, or $\left.C_{s}\right)$}

Spherical aberration is similar to chromatic aberration, except that the electrons have varying distances to the optical axis instead of varying energies, see Figure 4.11a. The electrons deviate differently due to the electromagnetic lenses fulfilling the four Scherzer conditions [169, 172]:

1. Rotational symmetry

2. Constant with respect to time

3. No charge on the optical axis

4. Creates a real image

As the focusing is independent on angle, the Gaussian surface will appear bowl-like, see Figure 4.11. Spherical aberration is the main resolution limiting aberration in standard TEMs.
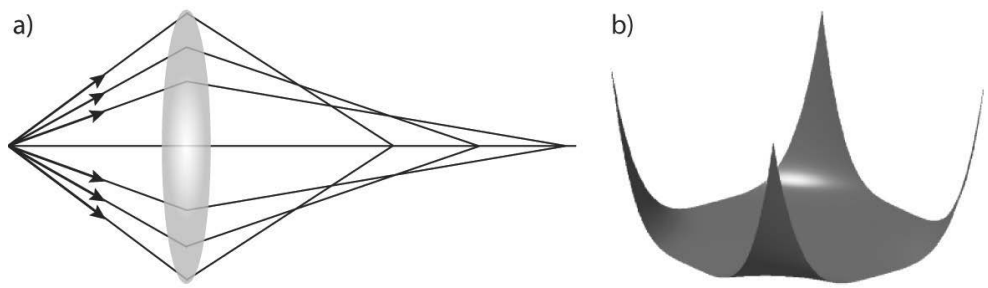

Figure 4.11. Spherical aberration causes the electrons to focus at different positions depending on initial radii (a), which causes a bowl-like shape of the electron wave (b).

\subsubsection{Astigmastim $\left(C_{1,2}\right.$ or $\left.A_{2}\right)$}

Astigmatism originates from either a non-perfect rotationally symmetric lens, which causes the focus to vary with azimuthal angle, see Figure 4.12 .
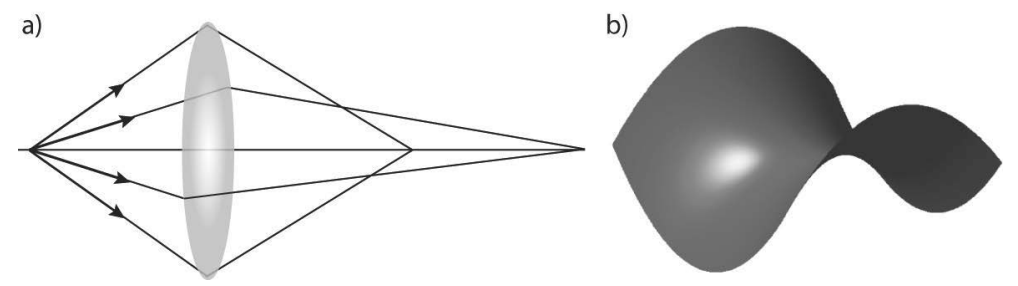

Figure 4.12. Astigmatism causes the focal point to vary with azimuthal angle (a) which is seen as a saddle-like appearance of the Gaussian surface (b). 


\subsubsection{Coma $\left(C_{2,1}\right.$ or $\left.B_{2}\right)$}

Coma is due to electrons originating away from the optical axis, causing the electrons to focus off the optical axis, see Figure 4.13.
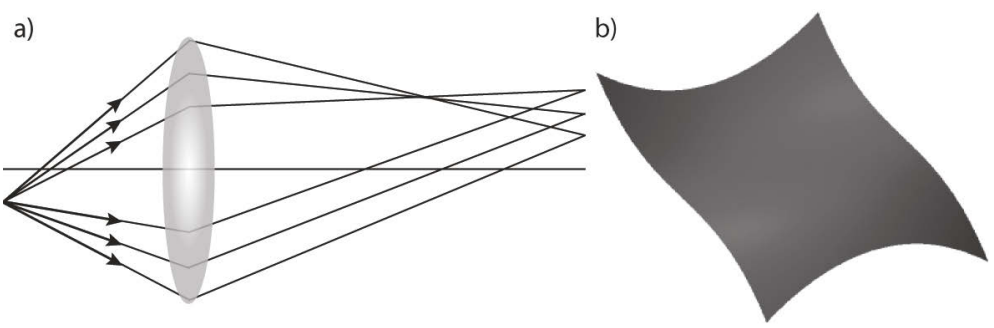

Figure 4.13. Coma cause the electrons to focus off-axis (a) and causes the Gaussian surface to appear 'wavy' (b).

\subsubsection{Correcting Aberrations}

\section{Correcting Chromatic Aberrations}

For atomic imaging and especially for high resolution spectroscopy, the energy resolution needs to be as high as possible. There are two methods to increase the energy resolution, either with a $C_{c}$ corrector, which removes the effect of the energy spread in a similar manner to aberration-correctors [173], or with a monochromator. A Wien-type monochromator spread the beam according to energy and employ a slit to select a part of the beam [174], see Figure 4.14. A monochromator reduces the energy spread to $\sim 180 \mathrm{meV}$ for the $\mathrm{Titan}^{3}$ and a chromatic corrector reduces the energy spread to $\sim 17 \mathrm{meV}$ for the $\mathrm{UltraSTEM}^{\mathrm{TM}}$.

As the sample thickness affect the amount of inelastic scattering, for imaging and spectroscopy at $60 \mathrm{kV}$ samples should be less than $100 \AA$ thick to reduce the chromatic aberration.
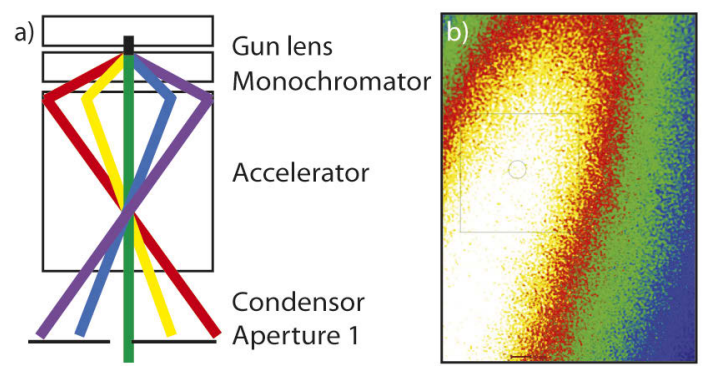

Figure 4.14. Schematic of a Wien-type monochromator (a) and an image of the elongated beam (b) where the different colors correspond to varying electron beam intensity. The energy changes when going along the elongated beam. 


\section{Correcting Other Aberrations}

In standard TEM astigmatism and coma can be corrected by introducing dipole lenses, which stretch the beam in two perpendicular directions. This introduces astigmatism in the other direction and the two astigmatisms cancel each other out. Aberration-corrected TEMs can correct up to sixth order aberrations $\left(C_{5,6}\right)$, thus reducing astigmatism, coma, and spherical aberration either in the probe (probe corrector) or in the CTEM image (image corrector). As the spherical aberration is reduced, these aberration-correctors are often referred to as $C_{s}$-correctors.
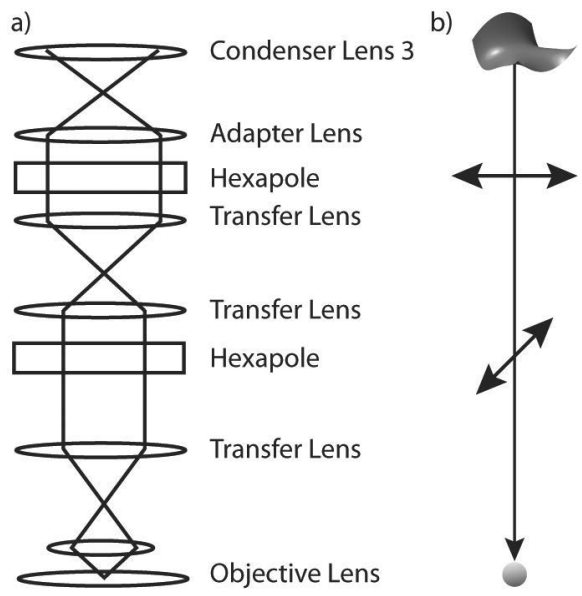

Figure 4.15. Schematic of a probe corrector (a), which removes aberrations by stretching the probe in two perpendicular directions (b).

The probe corrector and image corrector are based on the same principle. In the Titan ${ }^{3}$, they consist of a series of dipole and hexapole electromagnetic lenses, see Figure 4.15, which stretch the electron probe or beam in different directions and thus counteract aberrations from the electromagnetic lenses by introducing the same aberration but with opposite sign.

Aberration-correctors can also set specific values of the aberration coefficients, which is used in Negative $C_{s}$ Imaging (NCSI), where the spherical aberration is set to counteract a higher order spherical aberration. This increases the contrast for low-contrast materials [175].

\subsubsection{Stability of Aberration-Corrected TEM}

As the aberrations are minimized, the effect of the TEM surroundings on phase variations in high resolution imaging and spectroscopy become stronger. Air flows, temperature variations between different parts of the microscope and instabilities in the sample holder stage cause movements which can be detected [176]. External magnetic and electric fields affect spectroscopy measurements, as the spectrometer contains a sensitive magnetic prism [163]. 
To minimize these effects, the Linköping University's Titan ${ }^{3}$ is located inside a specially built laboratory, the Angström house, see Figure 4.16. The lab is designed to minimize ground vibrations, external electromagnetic fields, air flows and temperature gradients. This stable environment have enabled the instrument to reach $\sim 1 \AA$ point resolution and $70 \mathrm{meV}$ energy resolution at $60 \mathrm{kV}$, while for $300 \mathrm{kV}$ the microscope reaches $<0.6 \AA$ point resolution and $\lesssim 70 \mathrm{meV}$ energy resolution.

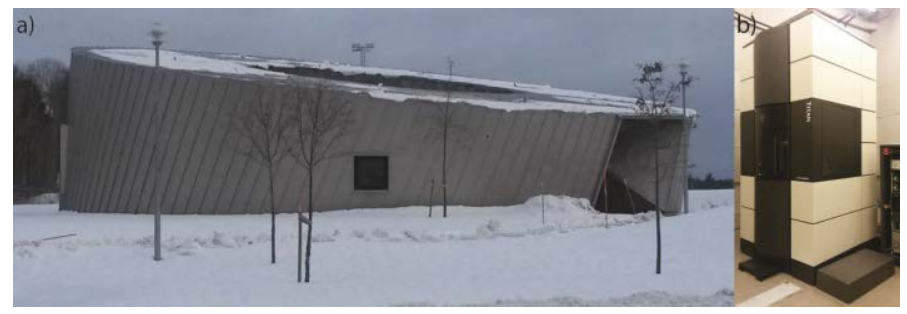

Figure 4.16. The Ångström House (a) in which the Linköping double-corrected Titan ${ }^{3}$ (b) is placed.

\subsection{Main Analytical TEM Techniques}

The basis of analytical TEM is the interaction between electrons and sample where the electron loses energy (inelastic scattering), see Figure 4.17. There are two main analytical methods employed in TEM, Energy Dispersive X-ray Spectroscopy (EDX) and EELS. EDX detects X-rays emitted from the sample while EELS measure the energy loss of the inelastic scattered electrons.

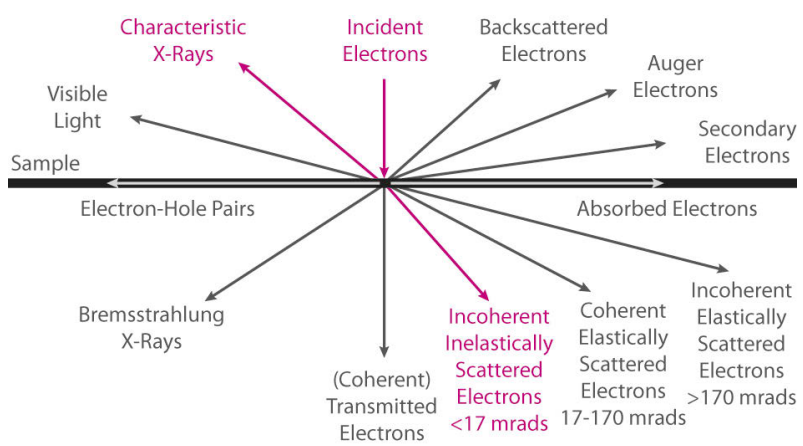

Figure 4.17. The most common interactions between electrons and sample. The pink text shows the interactions used for analytical TEM. 


\subsubsection{Inelastic Scattering}

Inelastic scattering occur through many types of interactions, depending on electron energy and sample properties. The most common inelastic interaction causes the electron cloud to oscillate, creating a temporary density variation (plasmon). Other interactions include heating, i.e. local vibration of the lattice (phonon), and ejection of electrons from the atom either from the conduction or valence band (secondary electrons) or the inner shell (Auger electrons). Shell electrons can also be excited to a higher energy state and as the electron re-enter an lower energy state, the excess energy is released as photons ( $X$-rays from inner shell electrons, visible light from outer shell electrons).

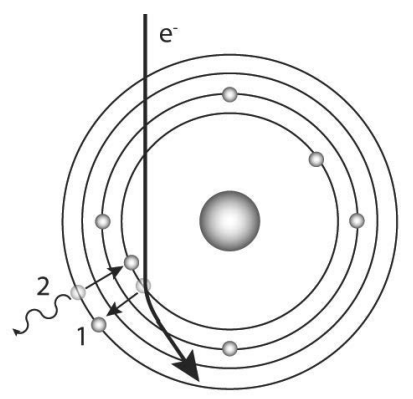

Figure 4.18. Schematic of inelastic scattering with the electron cloud causing an electron to move to a higher state (1). As the electron re-enters a lower state, radiation is emitted $(2)$.

\subsubsection{Electron Energy Loss Spectroscopy (EELS)}

EELS can measure many different interactions, see Table 4.1, and is most accurate for lower mass elements. The energy losses are measured by a spectrometer positioned at the end of the TEM column. Inside the spectrometer a magnetic prism deflects the electrons with respect to energy loss, and the electrons are subsequently detected by a CCD camera.

Table 4.1. Interactions which can be analyzed by EELS.

\begin{tabular}{ll}
\hline Interaction & Energy Loss $(\mathbf{e V})$ \\
\hline Optical signals (infrared, visible, UV) & $<3$ \\
Inter- and intraband transition & $2-25$ \\
Plasmon excitation & $5-25$ \\
Inner shell ionization & $\sim 50-2000$ \\
\hline
\end{tabular}

A typical EEL spectrum is shown in Figure 4.19. The spectrum can be divided into three regions; the zero loss region, Figure 4.19a, containing electrons trans- 
mitted with negligible energy loss, the low loss region, Figure 4.19b, containing electrons scattered by the valence or conductance band, and the core loss region, Figure $4.19 \mathrm{c}$, containing electrons scattered by core electrons.

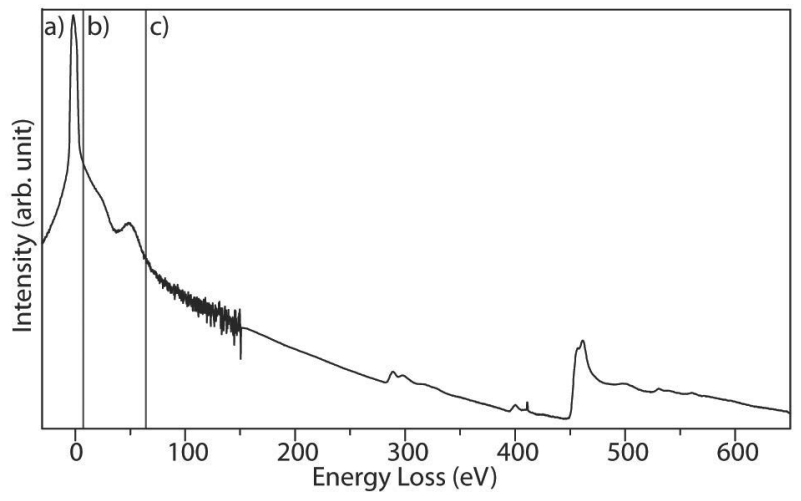

Figure 4.19. An EELS spectrum of $\mathrm{Ti}_{3} \mathrm{C}_{2} \mathrm{~T}_{x}$ MXene with the ZLP (a), low-loss (b), and core-loss (c) regions marked. The spectrum is shown in a logarithmic scale as the core loss signals are very low compared to the zero loss peak.

\section{Zero Loss Region}

The zero loss region consist of the Zero Loss Peak (ZLP) where the positive side is shaped by phonon interaction. The negative side arise from electrons tunneling from the FEG tip and the PSF of the detector. As the ZLP depends on the energy spread and coherence of the electrons, measuring the Full Width Half Maximum (FWHM) of the ZLP gives the energy resolution.

\section{Low Loss Region}

The low loss region includes losses up to about $50 \mathrm{eV}$ and originate from interactions with weakly bound electrons. In this energy range electronic information can be found, such as free-electron density, valence states, dielectric constant, band gap, and chemical bonds. As inelastic interaction increase with decreased electron energy, using $60 \mathrm{kV}$ acceleration voltage increases the low loss signal compared to $300 \mathrm{keV}[166]$.

The strongest features in the low loss region is the plasmon peak, seen in Figure $4.19 \mathrm{~b}$, which depends on sample chemistry. The position of the plasmon peak is determined by the samples electronic state and whether the plasmon occur on the surface (surface plasmons) or in the bulk (bulk plamons). Surface plasmons have about half the energy of a bulk plasmon [163] and surface plasmons of single $\mathrm{Ti}_{3} \mathrm{C}_{2} \mathrm{~T}_{x}$ MXene sheets were investigated in Paper $\mathbf{V}$. The bulk plasmon peak intensity can be used to determine sample thickness as the mean free path of a plasmon is in the order of $\sim 1000 \AA$. 
Infrared Spectroscopy by EELS Energy losses below a few eV results from induced motion of molecules, either vibrational, see Figure 4.20, or rotational. Vibrational motion is caused by movement of atoms within the molecule, while for rotational movement the entire molecule changes position [177]. As each molecule has a specific vibrational mode with a specific frequency, this method was used in Paper V to study MXene resting on amorphous carbon support. Intramolecular vibrations of water molecules intercalated between MXene sheets and stretching of $\mathrm{C}-\mathrm{H}$ bonds present in amorphous carbon could be resolved in the study.

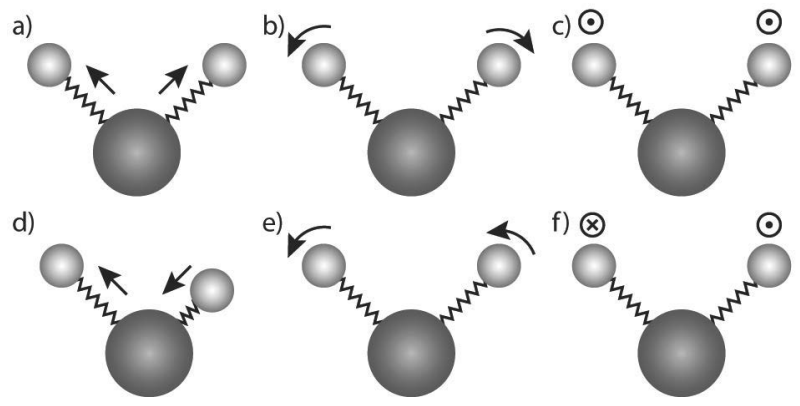

Figure 4.20. Molecular vibrations illustrated by a water molecule. The atoms oscillate by either stretching (a,d), bending (b), wagging (c), rocking (e), or twisting (f).

\section{Core loss region}

The core loss region originate from ionization of core shell electrons which have a binding energy $>50 \mathrm{eV}$ and provides information regarding chemical composition and electronic structure. The intensity of the core loss region is smaller than the other regions due to smaller interaction cross section.

The signals (edges) have an onset at the minimum energy required for ionization for a specific element and the structure depends on the samples electronic structure. At the first $\sim 50 \mathrm{eV}$ (Energy Loss Near Edge Structure (ELNES)), see Figure 4.21a, the shape is influenced by plasmons and the Density of States (DOS). The DOS is strongly affected by the bonding between atoms and plural scattering by nearby atoms. Investigation of the ELNES structure was employed in Paper III to investigate variations in chemical structure of individual $\mathrm{Ti}_{3} \mathrm{C}_{2} \mathrm{~T}_{x}$ sheets and in Paper I to investigate the bonding between transfer residues.

$\mathrm{Up}$ to hundreds of $\mathrm{eV}$ after the edge, the shape depends on electron diffraction by a single nearby atom (Extended Energy Loss Fine Structure (EXELFS)), see Figure $4.21 \mathrm{~b}$, which provide information about the atomic positions of atoms separated by a few nm.

The background results from plural, random scattering events and tails from earlier edges as well as variations in the spectrometer. Core Loss EELS of graphene and MXene show $\operatorname{sp}^{2}\left(\pi^{*}\right)$ and $\operatorname{sp}^{3}\left(\sigma^{*}\right)$ orbitals due to their different binding energies, see Figure 4.21. As can be seen in the figure, changes in bonding can be seen as a shift of the edge onset or a variation of the edge width. 


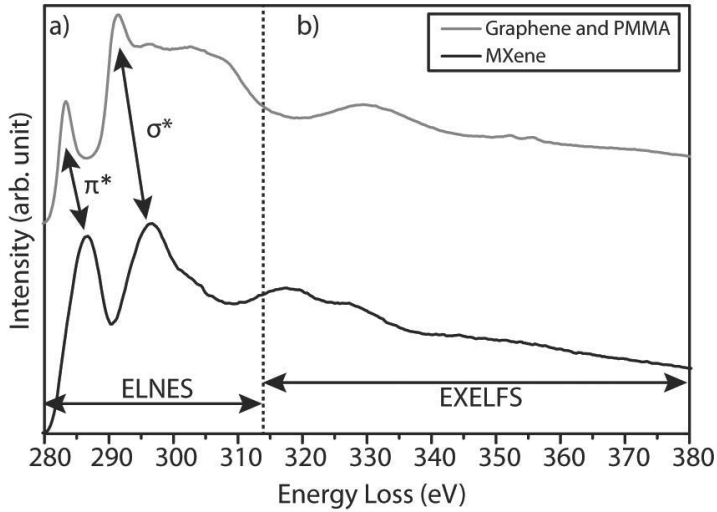

Figure 4.21. Comparison between in carbon edge for single sheet of $\mathrm{Ti}_{3} \mathrm{C}_{2} \mathrm{~T}_{x}$ MXene and graphene with PMMA residues. The ELNES structure is indicated in (a) and the EXELFS structure in (b).

\subsubsection{Spectrum Imaging}

EELS and EDX can be performed both in CTEM and STEM mode. In CTEM an average spectrum from the illuminated area is acquired while in STEM the signal originates from the small area where the probe is positioned. Combining STEM imaging and EELS results in a three-dimensional data cube (Spectrum Imaging $(S I)$, where each pixel correspond to one EEL spectrum, see Figure 4.22. This enable investigation of changes in a sample down to the $\AA$-scale in aberrationcorrected microscopes. Summing spectra in a SI increases the signal-to-noise ratio and enhance features in the spectra.

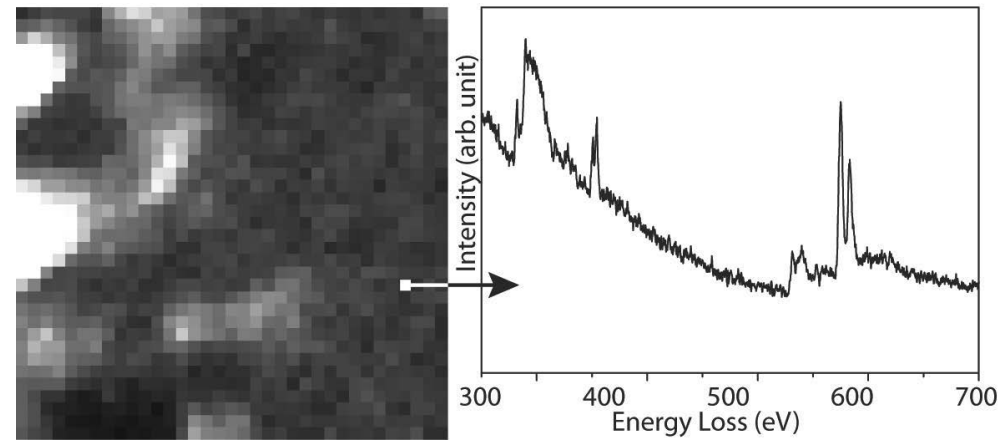

Figure 4.22. EELS Spectrum Image of Cr particles on graphene with PMMA residues. 


\subsection{Radiation Damage}

During TEM measurements the sample is damaged by the ionizing high energy electrons. The amount and type of damage depends on the electron energy, beam current, and the sample itself. If the energy of the incident electrons are above the binding energy of the material (damage threshold), chemical bonds are broken, which cause structural changes in the material.

Carbon based materials have low bond energies, $\mathrm{sp}^{3}$ bonds have $3.5 \mathrm{eV}$ and the $\mathrm{sp}^{2}$ bonds in graphene has a bond energy of $17 \mathrm{eV}$, while sp bonds have $36 \mathrm{eV}$ [166]. This corresponds to incident electron energies of 20, 80, and $220 \mathrm{keV}$ [178]. TEM analysis at 200-300 keV will thus damage the material.

The radiation damages affecting graphene and MXene with surface groups are knock-on displacement, sputtering, heating, and radiolysis.

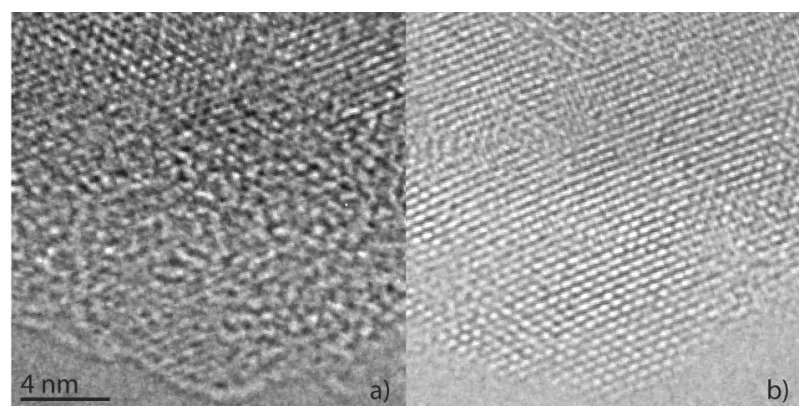

Figure 4.23. Radiation damage on $\mathrm{MXene} \mathrm{Ti}_{3} \mathrm{C}_{2} \mathrm{~T}_{x}$ from exposure to a $300 \mathrm{keV}$ electron beam, prior to exposure (a) and after exposure (b).

\subsubsection{Knock-on Displacement}

During elastic interaction the atom may be displaced from its lattice position. Atoms in bulk may move to an interstitial position while atoms on surfaces may start to diffuse on the surface $[179,180]$. Surface atoms are more easily displaced than bulk atoms due to their lower adsorption energy. Knock-on displacement is the main radiation damage in conducting samples, such as graphene [179] and MXene.

\subsubsection{Sputtering}

During knock-on displacement, the atoms can be ejected from the surface of a sample (sputtered). Sputtering occur on the beam exiting side of the sample due to the direction of momentum transfer between incident electrons and the sample [181]. 


\subsubsection{Heating}

Heating occurs due to the creation of phonons. Samples with high thermal conductivity can easily transport phonons which reduces damage, but samples with low thermal conductivity, e.g. polymers present on graphene after transfer, are severely damaged as the bonds are broken [181].

\subsubsection{Radiolysis}

Radiolysis is most dominant in organic solids, such as polymers. Energy is transferred to a molecule, causing the bonds within the molecule to break. This causes a change of both shape and size of the molecule. [181]

\subsubsection{Limiting Radiation Damage}

There are several methods which can be employed to reduce the radiation damage. Among the most common methods are reducing exposure time, lowering electron current and lowering electron energy [179]. Reducing the exposure time limits all types of radiation damages as the total amount of incident electrons is minimized. Lowering the beam current limits knock-on damage and heating [181]. Lowering electron energy reduces heating and eliminates knock-on damage if the energy of the incident electrons are below the material's damage threshold. Most elements have a threshold around tens of $\mathrm{keV}$ and thus an acceleration voltage of $60 \mathrm{kV}$ is enough to remove knock-on damage $[179,181]$. However, radiolysis increases as the energy decreases.

As aberration-corrected TEMs have higher beam currents than standard TEMs, samples are more damaged in the aberration-corrected TEMs when the microscopes are operated at the same acceleration voltage [179]. However, aberrationcorrected TEMs can lower the acceleration voltage to much lower energies than standard microscopes with sustained point resolution. 


\section{CHAPTER 5}

\section{Conclusions}

This was a triumph.

I'm making a note here: HUGE SUCCESS.

It's hard to overstate my satisfaction...

"Still Alive" by Ellen McLain and Jonathan Coulton

Portal Soundtrack (2007)

In this thesis, I have shown how residues and surface groups on two-dimensional materials interact and I have shown the atomic structure of these materials by lowvoltage, monochromated aberration-corrected TEM. Here I describe conclusions drawn from my research and how this contributes to the field of two-dimensional materials. My hope is that my research will help to improve the growth and transfer processes of two-dimensional materials, enabling larger control of material's properties, as well as highlighting the importance of how TEM samples are prepared and how they are analyzed in the TEM.

\subsection{Graphene}

One of the greatest challenges in graphene research is producing defect-free and clean graphene. Residues from transfer between substrates can not be removed by chemical etching or high temperature annealing. The residues, polymer residues from the polymer support and metal oxide particles from the growth substrate, are unevenly distributed on both surfaces of free-standing graphene, and interact through the graphene sheet during annealing. During high temperature annealing, the polymer residues also grow by dendritic-like growth into additional, randomly oriented, graphene sheets. 
The presence of these residues present a problem for the manufacturing of pristine graphene by CVD. In order to synthesize pristine graphene, other metal etchants would be suitable, as well as other polymers which are more easily removed.

Thus in the graphene part of my research, I can conclude that there still is much research needed before we can produce clean, defect-free graphene which can be transferred to any arbitrary substrate.

\subsection{MXene}

Similar to graphene, the surface of MXene is inherently covered by surface groups and intercalants originating from synthesis and handling of the material. The surface groups are oxygen-based while intercalants are mainly water. High resolution STEM combined with EELS revealed that these atoms and molecules are randomly positioned on the surface of MXene, but on single sheets exposed to vacuum they may dissociate, leaving areas of bare MXene. Due to the chemical bond between MXene and surface groups, the local electronic structure of each MXene sheet is altered by the surface groups.

I have also shown that depending on how MXene is oriented in the TEM, the apparent structure in TEM images vary substantially.

In conclusion, low-voltage, monochromated, aberration-corrected TEM is a powerful method for investigating the structure and chemistry of individual sheets of two-dimensional materials, such as MXene. 


\section{CHAPTER 6}

\section{Future Outlook}

... I want to see gamma rays!

I want to hear X-rays!

And I want to...

I want to smell dark matter! ...

Cylon model number One (Brother Cavil), Battlestar Galactia, Season 4, Episode 17

(No Hope) 2009

Although much research has been performed on two-dimensional materials, there are still many questions which have not been answered and would be worth pursuing. Here I've written a few which I think could be interesting to pursue.

\subsection{Graphene}

Graphene has rarely been reported as both defect-free and completely free from surface molecules on a large scale. Often graphene exhibit patches of clean areas surrounded by transfer residues or contamination from exposure to air. It would be interesting to determine whether there is a method to create completely residuefree graphene for TEM analysis.

\subsection{MXene}

During TEM analysis of MXene, I have noted that the material is more stable at lower electron energies than higher. At $300 \mathrm{kV}$, much of the surface groups are removed quickly, while they remain on the surface at $60 \mathrm{kV}$. It would be interesting 
to study radiation damage in single MXene sheets as well as layered MXenes. Does MXene become more stable as it becomes thicker, and what happens with the intercalants and surface groups? It has also been noted that MXene degrades after being stored, however nothing has been published on the long term stability of MXene sheets. Will $\mathrm{Ti}_{3} \mathrm{C}_{2} \mathrm{~T}_{x}$ MXene degrade into layers of $\mathrm{TiO}_{x}$ and amorphous carbon? Is degradation during storage dependent on number of layers and/or the elements present in MXene?

It would also be interesting to perform the investigations in this thesis to other MXenes than $\mathrm{Ti}_{3} \mathrm{C}_{2} \mathrm{~T}_{x}$ and on a large (more than $1 \mu \mathrm{m}^{2}$ ) single sheets, so the influence from other sheets is removed. 


\section{Bibliography}

[1] Moore, G. E. Cramming more components onto integrated circuits. Electronics 38, 114-117 (1965).

[2] Greene, J. E. Tracing the 5000-year recorded history of inorganic thin films from $\sim 3000$ BC to the early 1900s AD. Appl. Phys. Rev. 1, 041302 (2014).

[3] Price, M. R., Ovcharenko, A. \& Raeymaekers, B. Qualitative evaluation of ultra-thin multi-layer diamond-like carbon coatings using molecular dynamics nanoindentation simulations. Tribol. Lett. 62, 1-10 (2016).

[4] Akiyama, T. et al. Rewritable dual-layer phase-change optical disk utilizing a blue-violet laser. Jpn. J. Appl. Phys. 40, 1598-1603 (2001).

[5] EU. Commission recommendation of 18 October 2011 on the definition of nanomaterial (2011/696/EU). Off. J. Eur. Union 38-40 (2011).

[6] Lewicka, Z. A., Yu, W. W., Oliva, B. L., Contreras, E. Q. \& Colvin, V. L. Photochemical behavior of nanoscale $\mathrm{TiO}_{2}$ and $\mathrm{ZnO}$ sunscreen ingredients. J. Photochem. Photobiol. A 263, 24-33 (2013).

[7] Teli, M. \& Sheikh, J. Modified bamboo rayon-copper nanoparticle composites as antibacterial textiles. Int. J. Biol. Macromolec 61, 302-307 (2013).

[8] Kroto, H. W., Heath, J. R., O'Brien, S. C., Curl, R. F. \& Smalley, R. E. C60: Buckminsterfullerene. Nature 318, 162-163 (1985).

[9] Shirasaki, Y., Supran, G. J., Bawendi, M. G. \& Bulovic, V. Emergence of colloidal quantum-dot light-emitting technologies. Nat. Phot. 7, 13-23 (2013).

[10] Pérez-Juste, J., Pastoriza-Santos, I., Liz-Marzán, L. M. \& Mulvaney, P. Gold nanorods: Synthesis, characterization and applications. Coord. Chem. Rev. 249, 1870-1901 (2005). 
[11] Ke, M. Y. et al. Application of nanosphere lithography to LED surface texturing and to the fabrication of nanorod LED arrays. IEEE J. Sel. Topics Quantum Electron. 15, 1242-1249 (2009).

[12] Two-dimensional materials. http://www.nature.com/subjects/two-dimensional-materials. Nature Publishing Group (Retrieved 2015-11-16).

[13] Wallace, P. R. The band theory of graphite. Phys. Rev. 71, 622-634 (1947).

[14] Novoselov, K. S. et al. Electric field effect in atomically thin carbon films. Science 306, 666-669 (2004).

[15] Coleman, J. N. et al. Two-dimensional nanosheets produced by liquid exfoliation of layered materials. Science 331, 568-571 (2011).

[16] Naguib, M., Mochalin, V. N., Barsoum, M. W. \& Gogotsi, Y. 25th anniversary article: MXenes: A new family of two-dimensional materials. Adv. Mater. 26, 992-1005 (2014).

[17] Butler, S. Z. et al. Progress, challenges, and opportunities in two-dimensional materials beyond graphene. ACS Nano 7, 2898-2926 (2013).

[18] Novoselov, K. S. et al. Two-dimensional atomic crystals. Proc. Natl. Acad. Sci. U. S. A. 102, 10451-10453 (2005).

[19] Alem, N. et al. Atomically thin hexagonal boron nitride probed by ultrahighresolution transmission electron microscopy. Phys. Rev. B 80, 155425 (2009).

[20] Novoselov, K. S. et al. A roadmap for graphene. Nature 490, 192-200 (2012).

[21] Zhu, F.-f. et al. Epitaxial growth of two-dimensional stanene. Nat Mater 14, 1020-1025 (2015).

[22] Kara, A. et al. A review on silicene - New candidate for electronics. Surf. Sci. Rep. 67, 1-18 (2012).

[23] Acun, A. et al. Germanene: the germanium analogue of graphene. J. Phys. Condens. Matter 27, 443002 (2015).

[24] Sofo, J. O., Chaudhari, A. S. \& Barber, G. D. Graphane: A two-dimensional hydrocarbon. Phys. Rev. B 75, 153401 (2007).

[25] Liu, H. et al. Phosphorene: an unexplored 2D semiconductor with a high hole mobility. ACS Nano 8, 4033-4041 (2014).

[26] Dean, C. R. et al. Boron nitride substrates for high-quality graphene electronics. Nat. Nanotechnol. 5, 722-726 (2010).

[27] Geim, A. K. Graphene: Status and prospects. Science 324, 1530-1534 (2009). 
[28] Lukatskaya, M. R. et al. Cation intercalation and high volumetric capacitance of two-dimensional titanium carbide. Science 341, 1502-1505 (2013).

[29] Elias, D. C. et al. Control of graphene's properties by reversible hydrogenation: evidence for graphane. Science 323, 610-613 (2009).

[30] Fashandi, H. et al. Dirac points with giant spin-orbit splitting in the electronic structure of two-dimensional transition-metal carbides. Phys. Rev. B 92, $155142(2015)$.

[31] Yang, K., Feng, L. \& Liu, Z. The advancing uses of nano-graphene in drug delivery. Expert Opin. Drug Deliv. 12, 601-612 (2015).

[32] Nicolosi, V., Chhowalla, M., Kanatzidis, M. G., Strano, M. S. \& Coleman, J. N. Liquid exfoliation of layered materials 340, 1226419 (2013).

[33] Ghidiu, M., Lukatskaya, M. R., Zhao, M.-Q., Gogotsi, Y. \& Barsoum, M. W. Conductive two-dimensional titanium carbide 'clay' with high volumetric capacitance. Nature 516, 78-81 (2014).

[34] Chen, D., Feng, H. \& Li, J. Graphene oxide: Preparation, functionalization, and electrochemical applications. Chem. Rev. 112, 6027-6053 (2012).

[35] Dreyer, D. R., Park, S., Bielawski, W. \& Ruoff, R. S. The chemistry of graphene oxide. Chem. Soc. Rev. 39, 228-240 (2010).

[36] Cheng, G., Calizo, I. \& Hight Walker, A. R. Metal-catalyzed etching of graphene governed by metal-carbon interactions: A comparison of $\mathrm{Fe}$ and Cu. Carbon 81, 678-687 (2015).

[37] Wang, Y., Li, Z., Wang, J., Li, J. \& Lin, Y. Graphene and graphene oxide: biofunctionalization and applications in biotechnology. Trends. Biotechnol. 29, 205-212 (2011).

[38] Ghidiu, M. et al. Synthesis and characterization of two-dimensional $\mathrm{Nb}_{4} \mathrm{C}_{3}$ (MXene). Chem. Commun. (Camb). 50, 9517-20 (2014).

[39] Naguib, M. et al. MXene: A promising transition metal carbide anode for lithium-ion batteries. Electrochem. Commun. 16, 61-64 (2012).

[40] Tarascon, J.-M. M. \& Armand, M. Issues and challenges facing rechargeable lithium batteries. Nature 414, 359-367 (2001).

[41] Ruska, E. The development of the electron microscope and of electron microscopy. Rev. Mod. Phys. 59, 627-638 (1987).

[42] Freundlich, M. M. Origin of the Electron Microscope. Science 142, 185-188 (1963).

[43] Bianco, A. et al. All in the graphene family-A recommended nomenclature for two-dimensional carbon materials. Carbon 65, 1-6 (2013). 
[44] Soldano, C., Mahmood, A. \& Dujardin, E. Production, properties and potential of graphene. Carbon 48, 2127-2150 (2010).

[45] Malard, L., Pimenta, M., Dresselhaus, G. \& Dresselhaus, M. Raman spectroscopy in graphene. Phys. Rep. 473, 51-87 (2009).

[46] Yanagi, K., Miyata, Y. \& Kataura, H. Optical and conductive characteristics of metallic single-wall carbon nanotubes with three basic colors; cyan, magenta, and yellow. Appl. Phys. Express 1, 0340031-0340033 (2008).

[47] Yang, Z.-P. et al. Experimental observation of an extremely dark material made by a low-density nanotube array. Nano Lett. 8, 446-451 (2008).

[48] Klein, C. A. \& Cardinale, G. F. Young's modulus and Poisson's ratio of CVD diamond. Diam. Relat. Mater. 2, 918-923 (1993).

[49] Blakslee, O. L., Proctor, D. G., Seldin, E. J., Spence, G. B. \& Weng, T. Elastic constants of compression-annealed pyrolytic graphite. J. Appl. Phys. 41, 3373-3382 (1970).

[50] Lee, C., Wei, X., Kysar, J. W. \& Hone, J. Measurement of the elastic properties and intrinsic strength of monolayer graphene. Science 321, 385388 (2008).

[51] Krishnan, A., Dujardin, E., Ebbesen, T., Yianilos, P. N. \& Treacy, M. M. J. Young's modulus of single-walled nanotubes. Phys. Rev. B 58, 14013-14019 (1998).

[52] Nordling, C. \& Österman, J. Physics Handbook for science and engineering (Studentlitteratur, 2004), seventh edn.

[53] Balandin, A. a. et al. Superior thermal conductivity of single-layer graphene. Nano Lett. 8, 902-907 (2008).

[54] Berber, S., Kwon, Y.-K. \& Tomanek, D. Unusually high thermal conductivity of carbon nanotubes. Phys. Rev. Lett. 84, 4613-4616 (2000).

[55] Isberg, J. et al. High carrier mobility in single-crystal plasma-deposited diamond. Science 297, 1670-1672 (2002).

[56] Klein, C. A. \& Straub, W. D. Carrier densities and mobilities in pyrolytic graphite. Phys. Rev. 123, 1581-1583 (1961).

[57] Durkop, T., Getty, S. A., Cobas, E. \& Fuhrer, M. S. Extraordinary mobility in semiconducting carbon nanotubes. Nano Lett. 4, 35-39 (2004).

[58] May, P. W. Diamond thin films: a 21st-century material. Phil. Trans. R. Soc. A 358, 473-495 (2000).

[59] Brandt, N., Chudinov, S. \& Ponomarev, Y. Semimetals: 1. Graphite and its compounds, vol. 20.1 (Elsevier, 2012, ISBN: 0-444-87049-0 (v. 1)). 
[60] Baughman, R. H., Zakhidov, A. A. \& de Heer, W. A. Carbon nanotubes The route toward applications. Science 297, 787-792 (2002).

[61] The 2010 Nobel Prize in Physics-Press Release. http://www.nobelprize.org /nobel_prizes/physics/laureates/2010/press.html. Nobelprize.org. Nobel Media AB 2014 (Retrieved 2016-04-08).

[62] Strong, H. M. \& Chrenko, R. M. Diamond growth rates and physical properties of laboratory-made diamond. J. Phys. Chem. 75, 1838-1843 (1971).

[63] Hounsome, L. S. et al. Origin of brown coloration in diamond. Phys. Rev. B 73, 125203 (2006).

[64] Giovannetti, G. et al. Doping graphene with metal contacts. Phys. Rev. Lett. 101, 4-7 (2008).

[65] Denis, P. A. Band gap opening of monolayer and bilayer graphene doped with aluminium, silicon, phosphorus, and sulfur. Chem. Phys. Lett. 492, 251-257 (2010).

[66] Liu, X. et al. Bonding and charge transfer by metal adatom adsorption on graphene. Phys. Rev. B 83, 235411 (2011).

[67] Ni, Z. H. et al. Raman spectroscopy of epitaxial graphene on a SiC substrate. Phys. Rev. B 77, 115416 (2008).

[68] Chae, S. J. et al. Synthesis of large-area graphene layers on poly-nickel substrate by chemical vapor deposition: wrinkle formation. Adv. Mater. 21, 2328-2333 (2009).

[69] Li, X. et al. Large-area synthesis of high-quality and uniform graphene films on copper foils. Science 324, 1312-1314 (2009).

[70] Zhou, S. Y. et al. Substrate-induced bandgap opening in epitaxial graphene. Nat. Mater. 6, 770-775 (2007).

[71] Liu, L., Qing, M., Wang, Y. \& Chen, S. Defects in graphene: Generation, healing, and their effects on the properties of graphene: A review. J. Mater. Sci. Technol. 31, 599-606 (2015).

[72] Yoon, H. J. et al. Carbon dioxide gas sensor using a graphene sheet. Sensor. Actuat. B-Chem. 157, 310-313 (2011).

[73] Johnson, J. L., Behnam, A., Pearton, S. J. \& Ural, A. Hydrogen sensing using Pd-functionalized multi-layer graphene nanoribbon networks. Adv. Mater. 22, 4877-4880 (2010).

[74] Li, W. et al. Reduced graphene oxide electrically contacted graphene sensor for highly sensitive nitric oxide detection. ACS Nano 5, 6955-6961 (2011). 
[75] Lee, J.-h., Loya, P. E., Lou, J. \& Thomas, E. L. Dynamic mechanical behavior of multilayer graphene via supersonic projectile penetration. Science 346, 1092-1096 (2014).

[76] Riedl, C., Coletti, C. \& Starke, U. Structural and electronic properties of epitaxial graphene on $\mathrm{SiC}(0001)$ : a review of growth, characterization, transfer doping and hydrogen intercalation. J. Phys. D. Appl. Phys. 43, 374009 (2010).

[77] Whitener, K. E. \& Sheehan, P. E. Graphene synthesis. Diam. Relat. Mater. 46, 25-34 (2014).

[78] Kobayashi, T. et al. Production of a 100-m-long high-quality graphene transparent conductive film by roll-to-roll chemical vapor deposition and transfer process. Appl. Phys. Lett. 102, 023112 (2013).

[79] Pirkle, a. et al. The effect of chemical residues on the physical and electrical properties of chemical vapor deposited graphene transferred to $\mathrm{SiO}_{2}$. Appl. Phys. Lett. 99, 10-13 (2011).

[80] Lupina, G. et al. Residual metallic contamination of transferred chemical vapor deposited graphene. ACS Nano 9, 4776-4785 (2015).

[81] Regmi, M., Chisholm, M. F. \& Eres, G. The effect of growth parameters on the intrinsic properties of large-area single layer graphene grown by chemical vapor deposition on Cu. Carbon 50, 134-141 (2012).

[82] Mattevi, C., Kim, H. \& Chhowalla, M. A review of chemical vapour deposition of graphene on copper. J. Mater. Chem. 21, 3324-3334 (2011).

[83] Kim, K. S. et al. Large-scale pattern growth of graphene films for stretchable transparent electrodes. Nature 457, 706-710 (2009).

[84] Kim, J. et al. Low-temperature synthesis of large-area graphene-based transparent conductive films using surface wave plasma chemical vapor deposition. Appl. Phys. Lett. 98, 091502 (2011).

[85] Ding, G. et al. Chemical vapor deposition of graphene on liquid metal catalysts. Carbon 53, 321-326 (2013).

[86] Harper, C. A. \& Petrie, E. M. Plastics materials and processes: A concise encyclopedia (John Wiley \& Sons, 2003, ISBN: 978-0-471-45920-0).

[87] Borin Barin, G. et al. Optimized graphene transfer: Influence of polymethylmethacrylate (PMMA) layer concentration and baking time on graphene final performance. Carbon 84, 82-90 (2015).

[88] Li, X. et al. Transfer of large-area graphene films for high-performance transparent conductive electrodes. Nano Lett. 9, 4359-4363 (2009). 
[89] Choi, W. et al. Effect of annealing in $\mathrm{Ar} / \mathrm{H}_{2}$ environment on chemical vapor deposition-grown graphene transferred with poly (methyl methacrylate) $\mathbf{1 4}$, 70-74 (2015).

[90] Lin, Y. C. et al. Graphene annealing: How clean can it be? Nano Lett. 12, 414-419 (2012).

[91] Kumar, K., Kim, Y.-S. \& Yang, E.-H. The influence of thermal annealing to remove polymeric residue on the electronic doping and morphological characteristics of graphene. Carbon 65, 35-45 (2013).

[92] Xie, W., Weng, L.-T., Ng, K. M., Chan, C. K. \& Chan, C.-M. Clean graphene surface through high temperature annealing. Carbon 94, 740-748 (2015).

[93] Liu, N. et al. The origin of wrinkles on transferred graphene. Nano Res. 4, 996-1004 (2011).

[94] Suk, J. W. et al. Transfer of CVD-grown monolayer graphene onto arbitrary substrates. ACS Nano 5, 6916-6924 (2011).

[95] Brundle, C. R., Evans, Jr., C. A. \& Wilson, S. Encyclopedia of materials characterization, surfaces, interfaces, thin films (Manning Publications Co., 1992, ISBN: 0-7506-9168-9), first edn.

[96] van Huis, M. A. et al. Atomic imaging of phase transitions and morphology transformations in nanocrystals. Adv. Mater. 21, 4992-4995 (2009).

[97] Holland, B. J. \& Hay, J. N. The effect of polymerisation conditions on the kinetics and mechanisms of thermal degradation of PMMA. Polym. Degrad. Stab. 77, 435-439 (2002).

[98] Zeng, W. R., Li, S. F. \& Chow, W. K. Review on chemical reactions of burning poly(methyl methacrylate) PMMA. J. Fire Sci. 20, 401-433 (2002).

[99] Kashiwagi, T. et al. Effects of weak linkages on the thermal and oxidative degradation of poly(methyl methacrylates). Macromolecules 19, 2160-2168 (1986).

[100] Huang, L.-W. et al. Characterization of the cleaning process on a transferred graphene. J. Vac. Sci. Technol. A 32, 050601 (2014).

[101] Zan, R., Bangert, U., Ramasse, Q. \& Novoselov, K. S. Metal-graphene interaction studied via atomic resolution scanning transmission electron microscopy. Nano Lett. 11, 1087-1092 (2011).

[102] Ramasse, Q. et al. Direct experimental evidence of metal-mediated etching of suspended graphene. ACS Nano 6, 4063-4071 (2012).

[103] Zan, R., Bangert, U., Ramasse, Q. \& Novoselov, K. S. Interaction of metals with suspended graphene observed by transmission electron microscopy. $J$. Phys. Chem. Lett. 3, 953-958 (2012). 
[104] Booth, T. J. et al. Macroscopic graphene membranes and their extraordinary stiffness. Nano Lett. 8, 2442-2446 (2008).

[105] Sun, Z. et al. Growth of graphene from solid carbon sources. Nature 468, 549-552 (2010).

[106] Hurle, D. T. Handbook of crystal growth 1, Fundamentals part B: Transport and stability (North-Holland, Elsevier Science Publishers, 1993, ISBN: 0444-88908-6), first edn.

[107] Gonser, B. W. Titanium alloys. Ind. Eng. Chem. 42, 222-226 (1950).

[108] Titanium (Ti). http://global.britannica.com/science/titanium. Encyclopaedia Britannica Online (Retrieved 2016-03-03).

[109] Naguib, M. et al. Two-dimensional nanocrystals produced by exfoliation of $\mathrm{Ti}_{3} \mathrm{AlC}_{2}$. Adv. Mater. 23, 4248-4253 (2011).

[110] Naguib, M. et al. Two-dimensional transition metal carbides. ACS Nano 6, 1322-1331 (2012).

[111] Naguib, M. et al. New two-dimensional niobium and vanadium carbides as promising materials for Li-ion batteries. J. Am. Chem. Soc. 135, 1596615969 (2013).

[112] Meshkian, R. et al. Synthesis of two-dimensional molybdenum carbide, $\mathrm{Mo}_{2} \mathrm{C}$, from the gallium based atomic laminate $\mathrm{Mo}_{2} \mathrm{Ga}_{2}$ C. Scr. Mater. 108, 147-150 (2015).

[113] Xu, C. et al. Large-area high-quality 2D ultrathin $\mathrm{Mo}_{2} \mathrm{C}$ superconducting crystals. Nat. Mater. 14, 1-8 (2015).

[114] Khazaei, M. et al. Novel electronic and magnetic properties of twodimensional transition metal carbides and nitrides. Adv. Funct. Mater. 23, 2185-2192 (2013).

[115] Xie, Y. \& Kent, P. R. C. Hybrid density functional study of structural and electronic properties of functionalized $\mathrm{Ti}_{n+1} \mathrm{X}_{n}(\mathrm{X}=\mathrm{C}, \mathrm{N})$ monolayers. Phys. Rev. B 87, 235441 (2013).

[116] Kurtoglu, M., Naguib, M., Gogotsi, Y. \& Barsoum, M. W. First principles study of two-dimensional early transition metal carbides. MRS Commun. 2, 133-137 (2012).

[117] Zhao, S., Kang, W. \& Xue, J. Manipulation of electronic and magnetic properties of $\mathrm{M}_{2} \mathrm{C}(\mathrm{M}=\mathrm{Hf}, \mathrm{Nb}, \mathrm{Sc}, \mathrm{Ta}, \mathrm{Ti}, \mathrm{V}, \mathrm{Zr}$ ) monolayer by applying mechanical strains. Appl. Phys. Lett. 104, 133106 (2014).

[118] Enyashin, A. N. \& Ivanovskii, A. L. Two-dimensional titanium carbonitrides and their hydroxylated derivatives: Structural, electronic properties and stability of MXenes $\mathrm{Ti}_{3} \mathrm{C}_{2-x} \mathrm{~N}_{x}(\mathrm{OH})_{2}$ from DFTB calculations. J. Solid State Chem. 207, 42-48 (2013). 
[119] Zha, X.-H. et al. Role of the surface effect on the structural, electronic and mechanical properties of the carbide MXenes. Europhys. Lett. 111, 26007 (2015).

[120] Halim, J. et al. X-ray photoelectron spectroscopy of select multi-layered transition metal carbides (MXenes). Appl. Surf. Sci. 362, 406-417 (2016).

[121] Dall'Agnese, Y., Rozier, P., Taberna, P.-L., Gogotsi, Y. \& Simon, P. Capacitance of two-dimensional titanium carbide (MXene) and MXene/carbon nanotube composites in organic electrolytes. J. Power Sources 306, 510-515 (2016).

[122] Shi, C. et al. Structure of nanocrystalline $\mathrm{Ti}_{3} \mathrm{C}_{2}$ MXene using atomic pair distribution function. Phys. Rev. Lett. 112, 125501 (2014).

[123] Wang, H.-W., Naguib, M., Page, K., Wesolowski, D. J. \& Gogotsi, Y. Resolving the structure of $\mathrm{Ti}_{3} \mathrm{C}_{2} \mathrm{~T}_{x}$ MXenes through multilevel structural modeling of the atomic pair distribution function. Chem. Mater. 28, 349-359 (2016).

[124] Xie, Y. et al. Role of surface structure on Li-ion energy storage capacity of two-dimensional transition-metal carbides. J. Am. Chem. Soc. 136, 63856394 (2014).

[125] Mauchamp, V. et al. Enhanced and tunable surface plasmons in twodimensional $\mathrm{Ti}_{3} \mathrm{C}_{2}$ stacks: Electronic structure versus boundary effects. Phys. Rev. B 89, 235428 (2014).

[126] Barsoum, M. W. \& Radovic, M. Elastic and mechanical properties of the MAX phases. Annu. Rev. Mater. Res. 41, 195-227 (2011).

[127] Wang, X. et al. Atomic-scale recognition of surface structure and intercalation mechanism of $\mathrm{Ti}_{3} \mathrm{C}_{2} \mathrm{X}$. J. Am. Chem. Soc. 137, 2715-2721 (2015).

[128] Miranda, A., Halim, J., Barsoum, M. W. \& Lorke, A. Electronic properties of freestanding $\mathrm{Ti}_{3} \mathrm{C}_{2} \mathrm{~T}_{x}$ MXene monolayers. Appl. Phys. Lett. 108, 033102 (2016).

[129] Halim, J. et al. Transparent conductive two-dimensional titanium carbide epitaxial thin films. Chem. Mater. 26, 2374-2381 (2014).

[130] Shein, I. R. \& Ivanovskii, A. L. Graphene-like nanocarbides and nanonitrides of d metals (MXenes): synthesis, properties and simulation. Micro. Nano. Lett. 8, 59-62 (2013).

[131] Shein, I. \& a.L. Ivanovskii. Planar nano-block structures $\mathrm{Ti}_{n+1} \mathrm{Al}_{0.5} \mathrm{C}_{n}$ and $\mathrm{Ti}_{n+1} \mathrm{C}_{n}(\mathrm{n}=1$, and 2) from MAX phases: Structural, electronic properties and relative stability from first principles calculations. Superlattice Microstruct. 52, 147-157 (2012). 
[132] Tang, Q., Zhou, Z. \& Shen, P. Are MXenes promising anode materials for Li ion batteries? Computational studies on electronic properties and Li storage capability of $\mathrm{Ti}_{3} \mathrm{C}_{2}$ and $\mathrm{Ti}_{3} \mathrm{C}_{2} \mathrm{X}_{2}(\mathrm{X}=\mathrm{F}, \mathrm{OH})$ monolayer. J. Am. Chem. Soc. 134, 16909-16916 (2012).

[133] Wang, S., Li, J. X., Du, Y. L. \& Cui, C. First-principles study on structural, electronic and elastic properties of graphene-like hexagonal $\mathrm{Ti}_{2} \mathrm{C}$ monolayer. Comput. Mater. Sci. 83, 290-293 (2014).

[134] Khazaei, M. et al. OH-terminated two-dimensional transition metal carbides and nitrides as ultralow work function materials. Phys. Rev. B 92, 075411 (2015).

[135] Hope, M. A. et al. Nmr reveals the surface functionalisation of $\mathrm{ti}_{3} \mathrm{c}_{2}$ mxene. Phys. Chem. Chem. Phys. 18, 5099-5102 (2016).

[136] Enyashin, A. N. \& Ivanovskii, A. L. Structural, electronic properties and stability of MX enes Ti $2 \mathrm{C}$ and Ti $3 \mathrm{C} 2$ functionalized by methoxy groups. J. Phys. Chem. C 117, 1-19 (2013).

[137] Eames, C. \& Islam, M. S. Ion intercalation into two-dimensional transitionmetal carbides: global screening for new high-capacity battery materials. $J$. Am. Chem. Soc. 136, 16270-6 (2014).

[138] Guo, X., Zhang, X., Zhao, S., Huang, Q. \& Xue, J. High adsorption capacity of heavy metal with two-dimensional MXenes: an ab initio study with molecular dynamics simulation. Phys. Chem. Chem. Phys. 18, 228-233 (2015).

[139] Ando, Y. \& Watanabe, S. First-principles study of metal-insulator control by ion adsorption on $\mathrm{Ti}_{2} \mathrm{C}$ MXene dioxide monolayers. Appl. Phys. Express 9, 015001 (2016).

[140] Lian, P. et al. Large reversible capacity of high quality graphene sheets as an anode material for lithium-ion batteries. Electrochim. Acta 55, 3909-3914 (2010).

[141] Mashtalir, O. et al. Intercalation and delamination of layered carbides and carbonitrides. Nat. Commun. 4, 1716 (2013).

[142] Li, Z. et al. Synthesis and thermal stability of two-dimensional carbide MXene $\mathrm{Ti}_{3} \mathrm{C}_{2}$. Mater. Sci. Eng. B 191, 33-40 (2015).

[143] Nair, R. R. et al. Spin-half paramagnetism in graphene induced by point defects. Nat. Phys. 8, 199-202 (2012).

[144] Khazaei, M., Arai, M., Sasaki, T., Estili, M. \& Sakka, Y. Two-dimensional molybdenum carbides: potential thermoelectric materials of the MXene family. Phys. Chem. Chem. Phys. 16, 7841-7849 (2014).

[145] Come, J. et al. A non-aqueous asymmetric cell with a $\mathrm{Ti}_{2} \mathrm{C}$-based twodimensional negative electrode. J. Electrochem. Soc. 159 (2012). 
[146] Dall'Agnese, Y. et al. High capacitance of surface-modified 2D titanium carbide in acidic electrolyte. Electrochem. Commun. 48, 118-122 (2014).

[147] Wang, X. et al. Pseudocapacitance of MXene nanosheets for high-power sodium-ion hybrid capacitors. Nat. Commun. 6, 6544 (2015).

[148] Wang, F. et al. An organ-like titanium carbide material (MXene) with multilayer structure encapsulating hemoglobin for a mediator-free biosensor. J. Electrochem. Soc. 162, B16-B21 (2015).

[149] Halim, J. et al. Synthesis and characterization of 2D molybdenum carbide (MXene). Adv. Func. Mater. DOI: 10.1002/adfm.201505328 (2016).

[150] Eklund, P., Beckers, M., Jansson, U., Högberg, H. \& Hultman, L. The $\mathrm{M}_{n+1} \mathrm{AX}_{n}$ phases: Materials science and thin-film processing. Thin Solid Films 518, 1851-1878 (2010).

[151] Harris, K. J., Bugnet, M., Naguib, M., Barsoum, M. W. \& Goward, G. R. Direct measurement of surface termination groups and their connectivity in the 2D MXene $\mathrm{V}_{2} \mathrm{CT}_{x}$ using NMR spectroscopy. J. Phys. Chem. C 119, 13713-13720 (2015).

[152] Lei, J.-C., Zhang, X. \& Zhou, Z. Recent advances in MXene: Preparation, properties, and applications. Front. Phys. 10, 276-286 (2015).

[153] Gan, L.-Y., Huang, D. \& Schwingenschlögl, U. Oxygen adsorption and dissociation during the oxidation of monolayer $\mathrm{Ti}_{2}$ C. J. Mater. Chem. A 43, 13672-13678 (2013).

[154] Enyashin, N. \& Ivanovskii, L. Atomic structure, comparative stability and electronic properties of hydroxylated $\mathrm{Ti}_{2} \mathrm{C}$ and $\mathrm{Ti}_{3} \mathrm{C}_{2}$ nanotubes. Comput. Theor. Chem. 989, 27-32 (2012).

[155] Magne, D., Mauchamp, V., Célérier, S., Chartier, P. \& Cabioc'h, T. Spectroscopic evidence in the visible-ultraviolet energy range of surface functionalization sites in the multilayer $\mathrm{Ti}_{3} \mathrm{C}_{2}$ MXene. Phys. Rev. B 91, 235428 (2015).

[156] Er, D., Li, J., Naguib, M., Gogotsi, Y. \& Shenoy, V. B. Ti C $_{2}$ MXene as a high capacity electrode material for metal ( $\mathrm{Li}, \mathrm{Na}, \mathrm{K}, \mathrm{Ca}$ ) ion batteries. ACS Appl. Mater. Interfaces 6, 11173-11179 (2014).

[157] Naguib, M., Unocic, R. R., Armstrong, B. L. \& Nanda, J. Large-scale delamination of multi-layers transition metal carbides and carbonitrides "MXenes". Dalton Trans. 44, 9353-9358 (2015).

[158] Peng, Q. et al. Unique lead adsorption behavior of activated hydroxyl group in two-dimensional titanium carbide. J. Am. Chem. Soc. 136, 4113-4116 (2014). 
[159] Xie, Y. et al. Prediction and characterization of MXene nanosheet anodes for non-lithium-ion batteries. ACS Nano 8, 9606-9615 (2014).

[160] Sun, D. et al. Structural transformation of MXene $\left(\mathrm{V}_{2} \mathrm{C}, \mathrm{Cr}_{2} \mathrm{C}\right.$, and $\left.\mathrm{Ta}_{2} \mathrm{C}\right)$ with $\mathrm{O}$ groups during lithiation: A first-principles investigation. ACS Appl. Mater. Interfaces 8, 74-81 (2016).

[161] Universale, C. O. Visual acuity measurement standard. Italian Journal of Ophthalmology II, 1-15 (1988).

[162] Pedrotti, F. L. \& Pedrotti, L. S. Introduction to optics (Prentice-Hall, Inc, 1993), second edn.

[163] Williams, D. B. \& Carter, C. B. Transmission electron microscopy: A textbook for materials science (Springer Science+Business Media, LLC, 2009, ISBN: 978-0-387-76502-0), second edn.

[164] The Nobel Prize in Chemistry 2014-Press Release. http://www.nobelprize.org/nobel_prizes/chemistry/laureates/2014/press.html. Nobelprize.org. Nobel Media AB 2014 (Retrieved 2016-04-07).

[165] Kawasaki, T. et al. Fine crystal lattice fringes observed using a transmission electron microscope with $1 \mathrm{MeV}$ coherent electron waves. Appl. Phys. Lett. 76, 1342-1344 (2000).

[166] Bachmatiuk, A. et al. Low voltage transmission electron microscopy of graphene. Small 11, 515-542 (2015).

[167] Kaiser, U. et al. Transmission electron microscopy at $20 \mathrm{kV}$ for imaging and spectroscopy. Ultramicroscopy 111, 1239-46 (2011).

[168] Krivanek, O. et al. High-energy-resolution monochromator for aberrationcorrected scanning transmission electron microscopy/electron energy-loss spectroscopy. Phil. Trans. R. Soc. 367, 3683-3697 (2009).

[169] Brydson, R. (ed.) Aberration-corrected analytical transmission electron microscopy (John Wiley \& Sons, Ltd, 2011, ISBN: 9780470518519).

[170] Krivanek, O., Dellby, N. \& Lupini, A. Towards sub- $\AA$ electron beams. Ultramicroscopy 78, 1-11 (1999).

[171] Haider, M., Uhlemann, S. \& Zach, J. Upper limits for the residual aberrations of a high-resolution aberration-corrected STEM. Ultramicroscopy 81, 163-175 (2000).

[172] Scherzer, O. The theoretical resolution limit of the electron microscope. J. Appl. Phys. 20, 20-29 (1949).

[173] Haider, M., Hartel, P., Müller, H., Uhlemann, S. \& Zach, J. Information transfer in a TEM corrected for spherical and chromatic aberration. Microsc. Microanal. 16, 393-408 (2010). 
[174] Tiemeijer, P., M.Bischoff, B.Freitag \& C.Kisielowski. Using a monochromator to improve the resolution in TEM to below $0.5 \AA$. Part I: Creating highly coherent monochromated illumination. Ultramicroscopy 114, 72-81 (2012).

[175] Urban, K. W. et al. Negative spherical aberration ultrahigh-resolution imaging in corrected transmission electron microscopy. Phil. Trans. R. Soc. A 367, 3735-3753 (2009).

[176] Muller, D. A. et al. Optimizing the environment for sub-0.2 nm scanning transmission electron microscopy 50, 219-226 (2001).

[177] Harris, D. C. Quantitative chemical analysis (W. H. Freeman and Company, 2007, ISBN: 9781429239899), seventh edn.

[178] Bell, D. C., Russo, C. J. \& Kolmykov, D. V. 40 keV atomic resolution TEM. Ultramicroscopy 114, 31-37 (2012).

[179] Egerton, R. F. Beam-induced motion of adatoms in the transmission electron microscope. Microsc. Microanal. 19, 479-486 (2013).

[180] Banhart, F. Irradiation effects in carbon nanostructures. Rep. Prog. Phys. 62, 1181-1221 (1999).

[181] Egerton, R., Li, P. \& Malac, M. Radiation damage in the TEM and SEM. Micron 35, 399-409 (2004). 


\section{Publications Included in the Thesis}

[I] Residue Reduction and Intersurface Interaction on Single Graphene Sheets

Linda H. Karlsson, Jens Birch, Aurelija Mockute, Arni S. Ingason, Huy Q. Ta, Mark H. Rummeli, Johanna Rosen, and Per O.Å. Persson Carbon, 100, 345-350 (2016).

[II] Graphene on Graphene Formation From PMMA Residues During Annealing

Linda H. Karlsson, Jens Birch, Aurelija Mockute, Arni S. Ingason, Huy Q. Ta, Mark H. Rummeli, Johanna Rosen, and Per O.Å. Persson Submitted

[III] Atomically Resolved Structural and Chemical Investigation of Single MXene Sheets

Linda H. Karlsson, Jens Birch, Joseph Halim, Michel W. Barsoum, and Per O.Å. Persson

Nano Letters, 15, 4955-4960 (2015).

[IV] High-Resolution TEM of Freestanding Delaminated $\mathbf{T i}_{n+1} \mathbf{C}_{n}$ MXenes

Ingemar Persson, Magnus Garbrecht, Linda H. Karlsson, Joseph Halim, Michel W. Barsoum, Justinas Palisaitis, and Per O.A. Persson Manuscript 
[V] Infrared Spectroscopy of $\mathbf{T i}_{3} \mathbf{C}_{2} \mathbf{T}_{x}$ MXene by High Resolution STEMEELS

Linda H. Karlsson, Fredrik S. Hage, Joseph Halim, Quentin M. Ramasse, Michel W. Barsoum, Jens Birch, and Per O.Å. Persson

Manuscript 


\section{CHAPTER 8}

\section{Summary of Included Papers}

\section{Paper I}

\section{Residue Reduction and Intersurface Interaction on Single Graphene Sheets}

\section{Summary}

PMMA residues and metal oxide particles present on opposite sides of a freestanding graphene sheet from the graphene transfer process are shown to interact through the graphene sheet during high temperature annealing. The reduction of PMMA residues are shown to depend on the metal oxide particle coverage.

\section{Author's Contribution}

Together with my main supervisor I planned the experiment and I performed the in situ annealing experiments. I performed data analysis with the help of my supervisors and wrote the paper together with the co-authors.

\section{Paper II}

Graphene on Graphene Formation From PMMA Residues During Annealing

\section{Summary}

PMMA residues from the transfer process of graphene is shown to decompose and grow as additional single graphene layers during high temperature annealing. 


\section{Author's Contribution}

Together with my main supervisor I planned the experiment and I performed the in situ annealing experiment, but not the atomic resolution imaging. I performed the data analysis with the help of my supervisors and I wrote the paper with minimal input from the co-authors.

\section{Paper III}

\section{Atomically Resolved Structural and Chemical Investigation of Single MXene Sheets}

\section{Summary}

Single sheets of $\mathrm{Ti}_{3} \mathrm{C}_{2}$ MXene are shown to be partially covered with surface groups, while surfaces of layered MXene sheets are completely covered. The surface groups are shown to be O-based and locally change the chemical structure of the MXene sheet.

\section{Author's Contribution}

I took part in planning and performing microscopy. Together with my main supervisor I performed the data analysis. I wrote the paper together with the co-authors.

\section{Paper IV}

High-Resolution TEM of Freestanding Delaminated $\mathbf{T i}_{n+1} \mathbf{C}_{n}$ MXenes

\section{Summary}

$\mathrm{Ti}_{3} \mathrm{C}_{2} \mathrm{~T}_{x}$ and $\mathrm{Ti}_{2} \mathrm{C}$ MXenes are shown at atomic resolution for different orientations. The atomic layering causes the MXene sheets to appear differently in high resolution TEM imaging depending on orientation in the TEM.

\section{Author's Contribution}

I contributed to the outline of the experiments and the experimental investigation, and commented the paper.

\section{Paper V}

Infrared Spectroscopy of $\mathrm{Ti}_{3} \mathrm{C}_{2} \mathbf{T}_{x}$ MXene by High Resolution STEMEELS 


\section{Summary}

Infrared spectroscopy of single $\mathrm{Ti}_{3} \mathrm{C}_{2} \mathrm{~T}_{x}$ MXene by STEM-EELS show that surface groups affect the surface plasmons of individual MXene sheets at energy losses below $1 \mathrm{eV}$. Intramolecular vibrations of water intercalated between MXene sheets are observed.

\section{Author's Contribution}

I performed the data analysis and wrote the paper with support from the coauthors. 


\section{CHAPTER 9}

\section{Publications Not Included in the Thesis}

[I] Optical Properties of CuCdTeO Thin Films Sputtered From CdTeCuO Composite Targets

A. Mendoza-Galván, G. Arreola-Jardón, L.H. Karlsson, P.O.Å. Persson, and S. Jiménez-Sandoval

Thin Solid Films, 571, 706-711 (2014).

[II] Atomically Resolved Microscopy of Ion Implantation Induced Dislocation Loops in $4 \mathrm{H}-\mathrm{SiC}$

L.H. Karlsson, A. Hallén, L. Hultman, and P.O.Å. Persson

Submitted 


\section{Papers}

The articles associated with this thesis have been removed for copyright reasons. For more details about these see:

http://urn.kb.se/resolve?urn=urn:nbn:se:liu:diva-127526 


\section{APPENDIX $A$}

\section{List of Figures}

1.1 Example of a thin film. . . . . . . . . . . . . . . . . 2

1.2 Schematic of a zero-dimensional C-60 molecule (a) and a one-dimensional carbon nanotube $(\mathrm{b}) \ldots \ldots \ldots 3$

1.3 Schematic of the two-dimensional materials graphene in top view (a) and side view (b), and $\mathrm{Ti}_{3} \mathrm{C}_{2}$ MXene in top view (c) and side view $(\mathrm{d}) \ldots \ldots \ldots \ldots \ldots$

2.1 The most common carbon allotropes: graphite (a), amorphous carbon (b), diamond (c), graphene (d,e), C-60 (f) and nanotube (g). (c) and (e) are TEM images, where (e) is filtered and has inverted contrast to simplify interpretation. . . . . . . . . . . . . .

2.2 Schematic of bernal stacking of graphite in side view (a) and top view (b), and turbostratic stacking of bilayer graphene in side view (c) and top view $(\mathrm{d}) \ldots \ldots \ldots \ldots \ldots$

2.3 The three different carbon bonds where the hybridized orbitals are shown in pink and the non-hybridized are shown in cyan; sp found in acetylene (a), $\mathrm{sp}^{2}$ in graphene (b), and $\mathrm{sp}^{3}$ in diamond (c). . . .

2.4 Schematic of the electronic structure of graphene with the position of the Fermi level indicated. . . . . . . . . . . . . . . .

2.5 Schematic of the CVD growth chamber where the heater encircles the chamber. . . . . . . . . . . . . . . 13

2.6 The transfer process of graphene between substrates. . . . . . . . . 13

2.7 Graphene with PMMA residues (black and grey) and metal oxide particles (white) after transfer to a TEM grid. . . . . . . . . . . 14 
2.8 Schematic of the magnetron sputtering process. The substrate is a representation of a graphene sheet transferred to a TEM heating chip (blue) with PMMA residues (lilac) on the surface opposite to the surface facing the target. . . . . . . . . . .

2.9 Schematic of a SEM. . . . . . . . . . . . . . . . .

2.10 A DENSsolution single tilt heating chip used in annealing studies of graphene, showing the front side (a), the back (b) and an optical microscopy image of $\operatorname{SiN}_{x}$ window with $\mathrm{Pt}$ wires and graphene (c) prior to annealing. . . . . . . . . . . . . .

2.11 Examples of dendritic growth of carbon. SEM image of dendritic growth (a) and STEM HAADF image of dendritic-like graphene (dark grey) growth from PMMA residues (light grey) on freestanding graphene (black) (b). . . . . . . . . . . . . .

3.1 Structure model of ideal $\mathrm{Ti}_{4} \mathrm{C}_{3}(\mathrm{a}, \mathrm{d}), \mathrm{Ti}_{3} \mathrm{C}_{2}$ (b,e) and $\mathrm{Ti}_{2} \mathrm{C}(\mathrm{c}, \mathrm{f})$ with indicated unit cells. . . . . . . . . . . . . . . .

3.2 Periodic table with elements of synthesized and predicted MXenes.

3.3 Structure of two MAX phases: $\mathrm{Ti}_{3} \mathrm{AlC}_{2}(\mathrm{a}, \mathrm{b})$ and $\mathrm{Ti}_{2} \mathrm{AlC}(\mathrm{c}, \mathrm{d}) \ldots$

3.4 Schematic of MXene synthesis from MAX phase by etching of the A-layer (a-c), which increases separation between sheets and introduces surface groups and intercalants (d). Crushing the resulting powder results in almost complete separation between sheets (e).

3.5 TEM of MXene at different magnifications: overview of holey carbon TEM grid with MXene particles (a), TEM image of a typical MXene flake resting on a carbon support (b), high resolution STEM HAADF image of the layering of MXene sheets shown from folded MXene where the diffuse areas are surface groups (c), and high resolution STEM HAADF image of a single MXene flake (d). . . .

3.6 Stable positions of surface groups on $\mathrm{Ti}_{3} \mathrm{C}_{2}$, shown in top view (a) and side view (b): atop, on top titanium atom, bridge, between two middle titanium and upper carbon, fcc, on top of middle titanium, and hcp, on top of carbon, as given by [153]. Note that the size of the surface groups are enlarged to increase visibility. . . . . . . .

3.7 Schematic of intercalated $\mathrm{Ti}_{3} \mathrm{C}_{2} \mathrm{~T}_{x}-\mathrm{OH}$ MXene (intercalants are shown in dark green). . . . . . . . . . . . . . . . . .

4.1 Schematic of the basic principle of a (S)TEM. Electrons are accelerated by an electron source, focused onto the sample by the objective lens and the transmitted electrons are detected. Lenses positioned along the optical axis control the illumination onto sample and detectors.

4.2 Schematic of a TEM column of an aberration-corrected microscope, showing the electron path from electron source to detector for both CTEM (left) and STEM (right) modes. Note the differences on the sample and detector. . . . . . . . . . . . . . . 
4.3 The most common interactions between electrons and sample (scattering angles from [163]). The pink text shows the interactions used for imaging in TEM. . . . . . . . . . . . . . . . . . . 34

4.4 Elastic scattering of an incident electron with an atom. . . . . . . . 34

4.5 The various STEM detectors (a) and examples of a STEM HAADF image (b) and a STEM BF image (c) of MXene $\mathrm{Ti}_{3} \mathrm{C}_{2} \mathrm{~T}_{x} . \ldots$. . .

4.6 Different contrast mechanisms in CTEM: thickness contrast of MXene flake on carbon support (a), BF diffraction contrast image of MXene (b), and phase contrast image of graphene (c) where the dark dots correspond to carbon atoms. . . . . . . . . . . .

4.7 Bragg diffraction of electrons by a MXene lattice. The incident angle and the scattering angle is the same. . . . . . . . .

4.8 Moiré pattern of two rotated two-dimensional sheets. Schematic with $25^{\circ}$ rotation (a) and $45^{\circ}$ rotation (b), a STEM HAADF image of bilayer graphene (c), and FFT pattern from (c) shown in (d) with indication of signals from the graphene layers. . . . . . . . . . .

4.9 Contrast values in the CTF $(T(u))$ as a function of lattice spacing $(u)$ of a standard microscope (FEI Tecnai) and the aberrationcorrected Titan $^{3}$ in CTEM mode. Point resolutions and information limits of the two microscopes are indicated. . . . . . . . . . .

4.10 Chromatic aberration causes electrons to focus at different positions depending on their energy. . . . . . . . . . . . . .

4.11 Spherical aberration causes the electrons to focus at different positions depending on initial radii (a), which causes a bowl-like shape of the electron wave (b). . . . . . . . . . . . . . .

4.12 Astigmatism causes the focal point to vary with azimuthal angle (a) which is seen as a saddle-like appearance of the Gaussian surface (b). 40

4.13 Coma cause the electrons to focus off-axis (a) and causes the Gaussian surface to appear 'wavy' (b). . . . . . . . . . . . .

4.14 Schematic of a Wien-type monochromator (a) and an image of the elongated beam (b) where the different colors correspond to varying electron beam intensity. The energy changes when going along the elongated beam.

4.15 Schematic of a probe corrector (a), which removes aberrations by stretching the probe in two perpendicular directions (b). . . . . . .

4.16 The Angström House (a) in which the Linköping double-corrected $\operatorname{Titan}^{3}(\mathrm{~b})$ is placed. . . . . . . . . . . . . . . . .

4.17 The most common interactions between electrons and sample. The pink text shows the interactions used for analytical TEM. . . . . .

4.18 Schematic of inelastic scattering with the electron cloud causing an electron to move to a higher state (1). As the electron re-enters a lower state, radiation is emitted $(2) \ldots \ldots \ldots$

4.19 An EELS spectrum of $\mathrm{Ti}_{3} \mathrm{C}_{2} \mathrm{~T}_{x}$ MXene with the ZLP (a), low-loss (b), and core-loss (c) regions marked. The spectrum is shown in a logarithmic scale as the core loss signals are very low compared to the zero loss peak. . . . . . . . . . . . . . . . . . 
4.20 Molecular vibrations illustrated by a water molecule. The atoms oscillate by either stretching $(\mathrm{a}, \mathrm{d})$, bending (b), wagging $(\mathrm{c})$, rocking (e), or twisting (f) . . . . . . . . . . . . . . . . 46

4.21 Comparison between in carbon edge for single sheet of $\mathrm{Ti}_{3} \mathrm{C}_{2} \mathrm{~T}_{x}$ MXene and graphene with PMMA residues. The ELNES structure is indicated in (a) and the EXELFS structure in (b) . . . . . . . . 47

4.22 EELS Spectrum Image of Cr particles on graphene with PMMA residues. . . . . . . . . . . . . . . . . .

4.23 Radiation damage on MXene $\mathrm{Ti}_{3} \mathrm{C}_{2} \mathrm{~T}_{x}$ from exposure to a $300 \mathrm{keV}$ electron beam, prior to exposure (a) and after exposure (b). . . . . 


\section{APPENDIX B}

List of Tables

2.1 Comparison of properties of some of the carbon allotropes. . . . . 9

4.1 Interactions which can be analyzed by EELS. . . . . . . . . . . . . 44 


\title{
APPENDIX C
}

Abbreviations

\author{
ADF Annular Dark Field \\ BCC Body-Centered Cubic \\ BF Bright Field \\ CTEM Conventional Transmission Electron Microscopy \\ CTF Contrast Transfer Function \\ CVD Chemical Vapour Deposition \\ DF Dark Field \\ DOS Density of States \\ EDX Energy Dispersive X-ray Spectroscopy \\ EELS Electron Energy Loss Spectroscopy \\ ELNES Energy Loss Near Edge Structure \\ EXELFS Extended Energy Loss Fine Structure \\ FCC Face Centered Cubic \\ FEGs Field Emission Guns \\ FFTs Fast Fourier Transforms \\ FWHM Full Width Half Maximum \\ HAADF High-Angle Annular Dark Field
}


HCP Hexagonal Close-Packed

LEDs Light Emitting Diodes

MAADF Medium-Angle Annular Dark Field

NCSI Negative $C_{s}$ Imaging

PMMA poly(methyl methacrylate)

PSF Point Spread Function

PVD Physical Vapor Deposition

SEM Scanning Electron Microscopy

SI Spectrum Imaging

STEM Scanning Transmission Electron Microscopy

TEM Transmission Electron Microscopy

TEM Transmission Electron Microscope

TMDs Transition Metal Dichalcogenides

VLM Visible Light Microscopes

XPS X-ray Photoelectron Spectroscopy

ZLP Zero Loss Peak 